\title{
Genetic manipulation of CNS cholesterol metabolism and its effects on cerebral $\beta$-amyloidosis
}

\author{
Dissertation \\ for the award of the degree \\ "Doctor rerum naturalium" \\ of the Georg-August University Göttingen \\ within the doctoral program Systems Neuroscience \\ of the Georg-August University School of Science (GAUSS) \\ submitted by \\ Jan Winchenbach \\ from Siegen
}

Göttingen 2018 


\section{Thesis committee}

Dr. Gesine Saher (Reviewer)

Department of Neurogenetics

Max-Planck-Institute of Experimental Medicine

Prof. Dr. Thomas Bayer (2 ${ }^{\text {nd }}$ Reviewer)

University Medical Center Göttingen

Division of Molecular Psychiatry

Prof. Dr. Swen Hülsmann

University Medical Center Göttingen

Experimental Neuroanesthesiology

\section{Extended thesis committee}

Prof. Dr. Ralf Heinrich

Department of Cellular Neurobiology

Schwann-Schleiden Research Center

Georg-August University Göttingen

Dr. Manuela Schmidt

Somatosensory Signaling Group

Max-Planck-Institute of Experimental Medicine

Dr. Katrin Willig

Nanoscale Microscopy and Physiology of the Brain

University Medical Center Göttingen and

Max-Planck-Institute of Experimental Medicine

Date of oral examination: 12.04.2018 


\section{Declaration}

I hereby declare that I prepared this Ph.D. thesis entitled "Genetic manipulation of CNS cholesterol metabolism and its effects on cerebral $\beta$-amyloidosis" independently and with no other sources and aids than quoted. The first two chapters of my result section and the corresponding methods are largely adapted from our manuscript "Inducible targeting of CNS astrocytes in Aldh1l1-CreERT2 BAC transgenic mice", published December 2016 in F1000 Research.

Göttingen, 28.02.2018

Jan Winchenbach 


\section{Acknowledgements}

I thank Prof. Klaus-Armin Nave Ph.D. for giving me the opportunity to work in his department and his support during the years of my Ph.D. work.

I am very grateful to Dr. Gesine Saher for her supervision. I want to thank her for the continuous support she has given me and her effort in creating a work group that I very much enjoyed being part of.

I would like to thank the members of my thesis committee, Prof. Dr. Thomas Bayer and Prof. Dr. Swen Hülsmann, for helpful discussions and their support during my graduation. I further thank the members of my examination board, Prof. Dr. Ralf Heinrich, Dr. Manuela Schmidt and Dr. Katrin Willig for taking part in my final examination.

I am very thankful to Prof. Dr. Swen Hülsmann for a great collaboration on our manuscript and his continuous friendly support in teaching me patch clamp recordings of astrocytes.

I want to thank Prof. Dr. Oliver Wirths and Dr. Melanie Hüttenrauch for helpful advice and Dr. Hans Klafki for his support and giving me the chance to work in his laboratory during $A \beta$ measurements.

I would like to thank Dr. Francesca Odoardi and Simone Hamann for their help with FACS analyses.

I want to express my deepest gratitude to my colleagues and friends, Sina Stumpf, Stefan Berghoff and Tim Düking for their support and valuable scientific discussions. I further thank Carolin Böhler and Silvia Thüne for valuable technical assistance.

I am thankful to Stefan Berghoff and Tim Düking for their contribution to this project by helping with BBB assessment and MACS analysis, respectively.

I want to thank Jana Kroll for valuable technical assistance and Borja Gómez Ramos for his contribution to this project during his lab rotation.

I am thankful to Dr. Katrin Kusch, Ulli Bode, Annette Fahrenholz, Torben Ruhwedel and Ramona Jung for very valuable technical support over the years. 
I thank Tanja Pawelz, Ines Malade, Cornelia Casper, Nadja Hoffmeister, Bianca Nickel and Dr. Ursula Fünfschilling for providing an excellent mouse house and transgenic animal facility.

I would like to thank Michaela Schmalstieg and Gabriele Endo for their help with administrative issues.

I am very grateful to all members of the Neurogenetics department for creating a friendly and scientifically fruitful working environment. I especially want to thank Ulrike Gerwig, Maria Eichel, Martin Meschkat, Torben Ruhwedel, Ramona Jung and Tilmann Unterbarnscheidt for making the work in the lab so much fun.

I want to express my deepest gratitude to my family for their constant support. I am especially grateful to Corinna Schlosser for her understanding and care during difficult times. 


\section{Content}

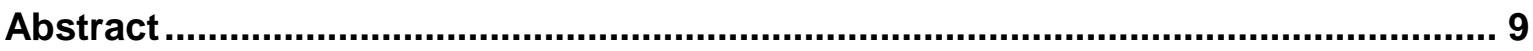

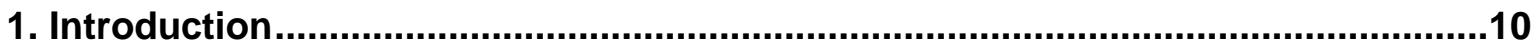

1.1 Peripheral cholesterol metabolism .............................................................. 10

1.2 Cholesterol metabolism in the brain............................................................. 11

1.2.1 Contribution of different cell types to brain cholesterol homeostasis................13

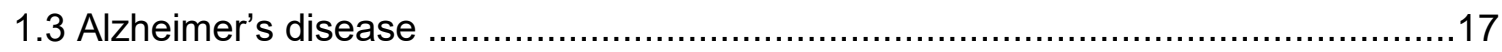

1.3.1 Role of cholesterol in Alzheimer's disease ..............................................18

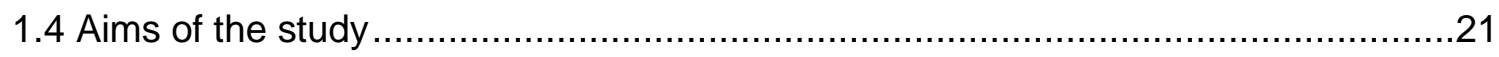

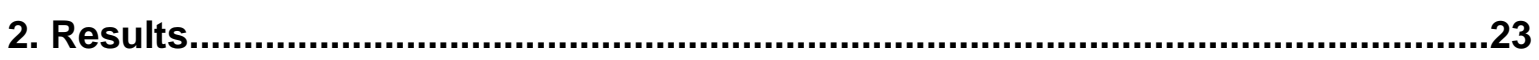

2.1 Are astrocytes efficiently targeted in Aldh1l1-CreERT2 transgenic mice? ...............23

2.2 What is the cellular specificity of Cre expression in Aldh1l1-CreERT2 mice? .........29

2.3 Conditional inactivation of cholesterol synthesis in astrocytes ..............................31

2.3.1 Do astrocytes survive that lack cell autonomous cholesterol synthesis? ..........34

2.3.2 What is the reason for reduced tdTomato signal in astroSQS-tdTo animals? ...35

2.3.3 Is squalene synthase successfully inactivated in astrocytes of conditional

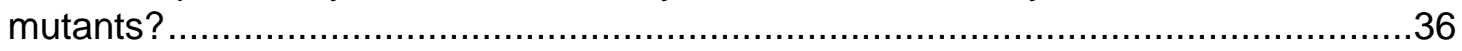

2.3.4 What are the electrophysiological properties of mutant astrocytes? .................38

2.3.5 Does lack of squalene synthase in astrocytes affect blood-brain barrier integrity?

2.4 Does lack of astrocytic cholesterol synthesis affect Alzheimer's disease pathology?

2.4.1 Are cholesterol homeostasis and BBB integrity altered in 5xFAD animals? ......43

2.4.2 Conditional inactivation of squalene synthase in 5XFAD animals .45

2.4.3 Does lack of astrocytic cholesterol synthesis affect cerebral $\beta$-amyloidosis?....46

2.4.4 Is the reduction in $A \beta$ deposition due to increased clearance by glial cells? .....48

2.4.5 Is the cholesterol metabolism altered in astroSQS-5XFAD mutants?................51

2.4.6 Is APP processing to generate $A \beta$ altered in astroSQS-5xFAD mutants? ........52

2.5 Does lack of neuronal cholesterol synthesis affect $A \beta$ production and deposition?.56

3. Discussion. .59

3.1 Efficient targeting of astrocytes in Aldh111-CreERT2 mice ..................................59

3.2 Inactivation of cholesterol synthesis in adult astrocytes ....................................60

3.3 Role of astrocytic cholesterol synthesis in cerebral $\beta$-amyloidosis ........................62

3.4 Role of neuronal cholesterol synthesis in cerebral $\beta$-amyloidosis ........................65

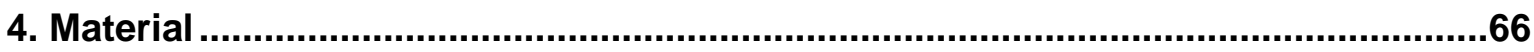

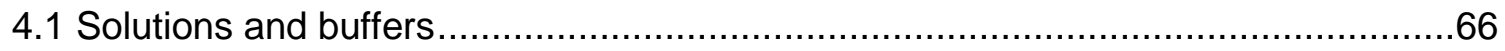

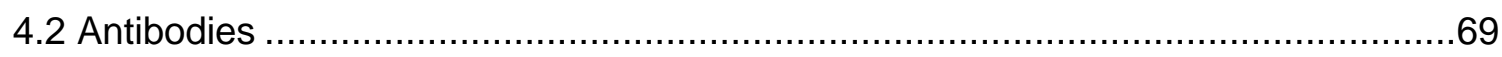




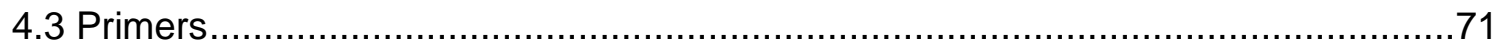

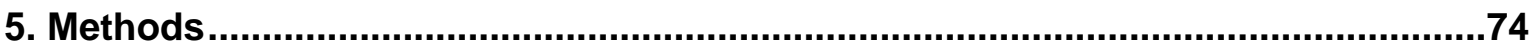

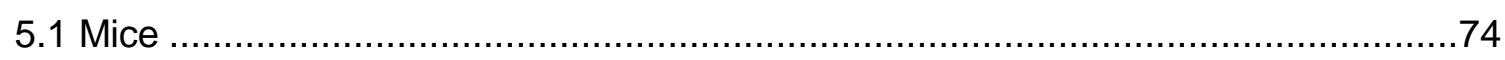

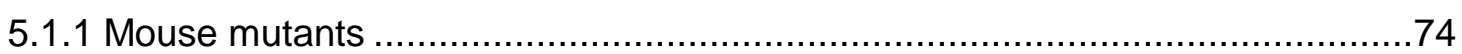

5.1.2 Generation of Aldh1l1-CreERT2 mice ................................................... 75

5.1.3 Generation of conditional Fdft1 mutants................................................... 75

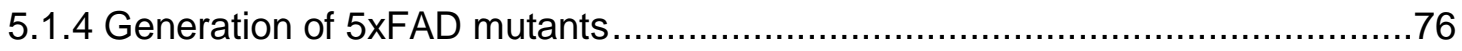

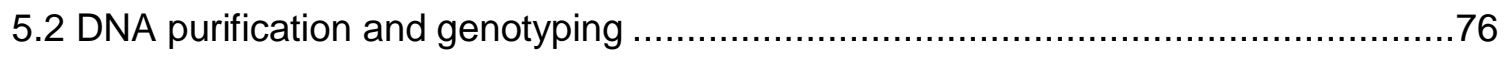

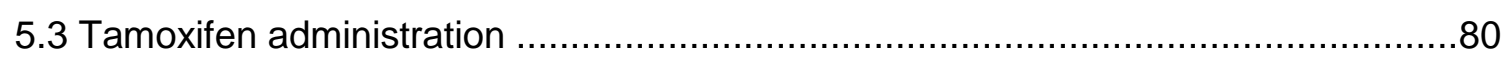

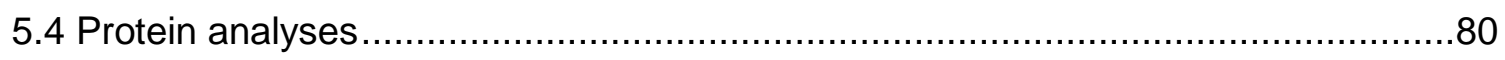

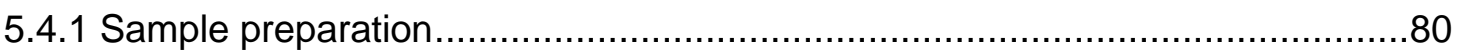

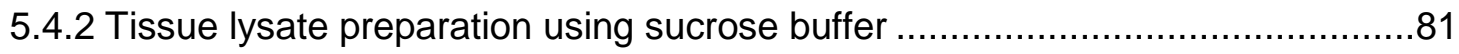

5.4.3 Tissue lysate preparation using TBS and SDS buffers ................................81

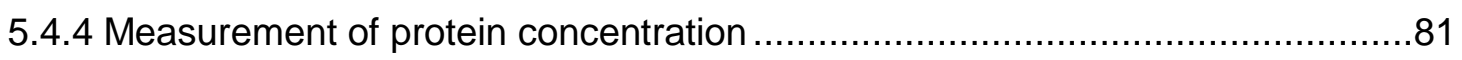

5.4.5 Protein separation using SDS-PAGE .................................................. 82

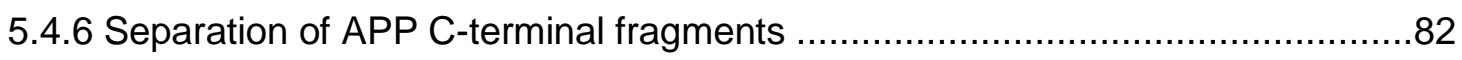

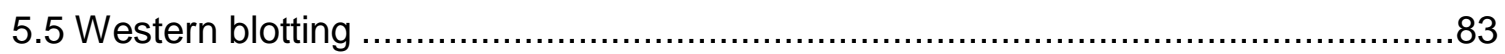

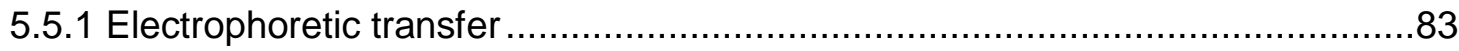

5.5.2 Immunodetection of proteins on PVDF membranes ....................................83

5.5.3 Densitometric quantification of immunoreactive bands .................................84

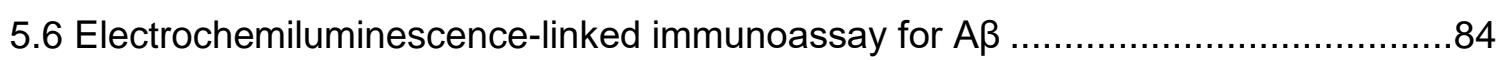

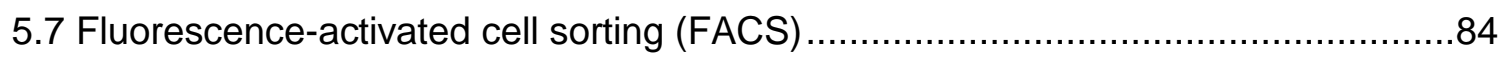

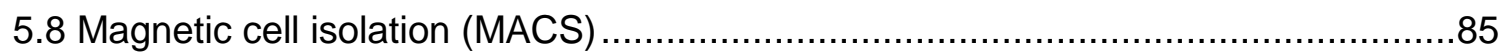

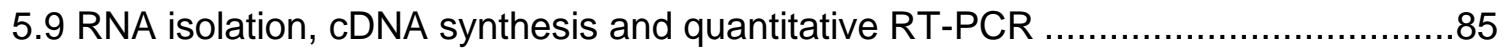

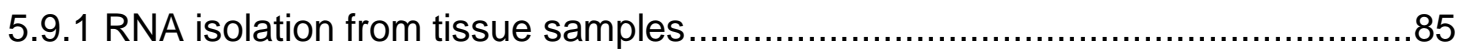

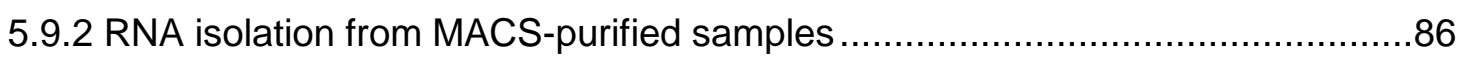

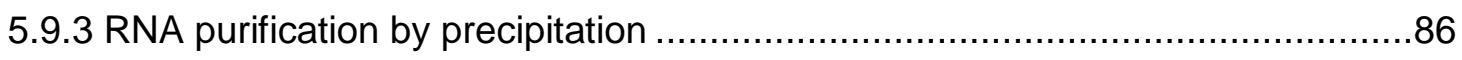

5.9.4 Complementary single stranded DNA (cDNA) synthesis .............................87

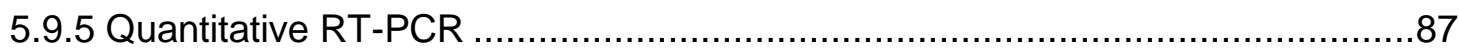

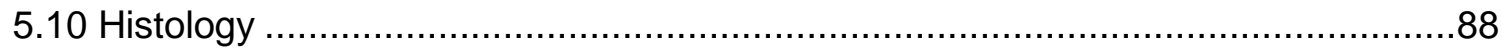

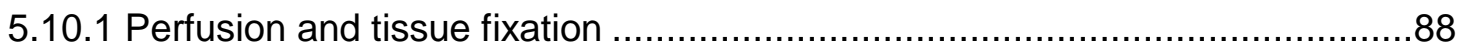

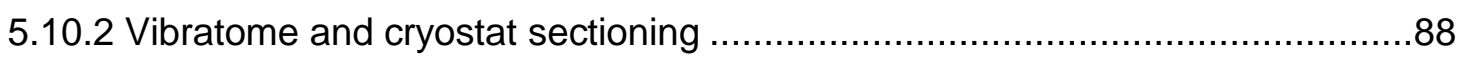

5.10.3 Paraffin embedding and microtome sectioning ........................................ 88

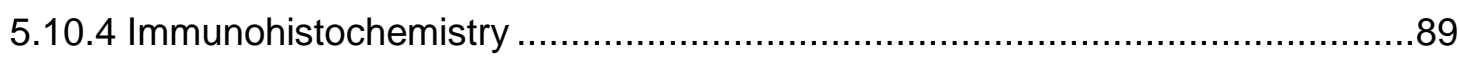

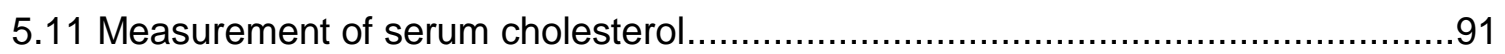

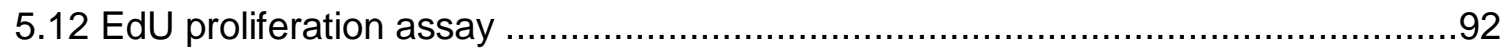

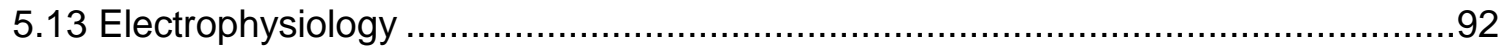

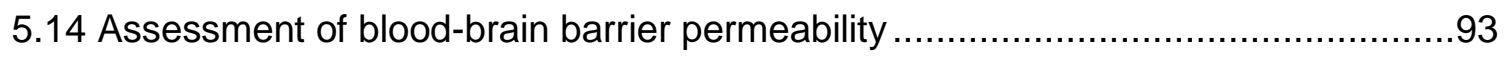




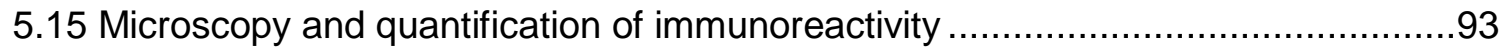

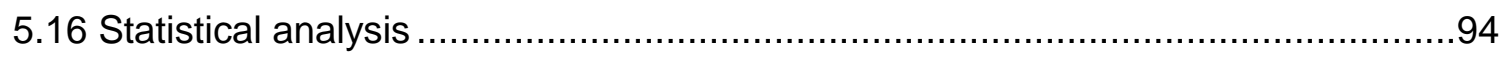

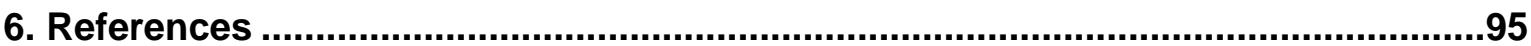




\section{Abstract}

Accumulating evidence implicates cholesterol metabolism in the pathogenesis of Alzheimer's disease (AD). However, the underlying mechanisms are not well understood. In the brain, cholesterol is synthesized locally by different cell types during development and in adulthood. The current understanding is that astrocytes are likely the major producers of cholesterol in the adult brain. However, in vivo evidence has been limited by the lack of genetic tools that allow efficient targeting of gene function in adult astrocytes. In this study, a newly generated BAC transgenic mouse line that expresses tamoxifen inducible Cre recombinase under control of the Aldh1l1 promoter was characterized. Analyses revealed that astrocytes in brain and spinal cord are targeted with high efficiency. Using this mouse line we inactivated cholesterol synthesis in adult astrocytes by targeting of squalene synthase (SQS), the enzyme catalyzing the first committed step in cholesterol biosynthesis. Conditional mutants did not show signs of brain pathology and mutant astrocytes were viable. Interestingly, albeit successful inactivation of SQS in astrocytes, brain cholesterol homeostasis was largely unaltered, suggesting compensatory efforts by other cell types. To address the role of astrocytic cholesterol synthesis in cerebral $\beta$-amyloidosis, we crossed conditional mutants with 5xFAD mice, an animal model of AD. In the hippocampus of these compound mutants, we found reduced deposition of $A \beta 42$ peptides accompanied by increased expression of Trem2 (triggering receptor expressed on myeloid cells 2). Although further investigation is required, Trem 2 could potentially facilitate $A \beta$ clearance by microglia cells in these mutants. Together, this study indicates that astrocytic cholesterol synthesis is not required in adult mice and underlines the importance of cholesterol metabolism in modulating cerebral $\beta$-amyloidosis. 


\section{Introduction}

\subsection{Peripheral cholesterol metabolism}

Cholesterol is an essential component of all animal cell membranes constituting 20$25 \%$ of plasma membrane lipids in most cells (Dietschy and Turley, 2004). Furthermore, cholesterol is the sole precursor of steroid hormones (Rone et al., 2009) and 7-dehydrocholesterol is involved in vitamin D production (reviewed in (Holick, 2007). Cholesterol is obtained from the diet and synthesized de novo in various organs. After uptake of cholesterol in the small intestine and esterification in enterocytes, triglycerides and esterified cholesterol are transported together in lipoprotein particles called chylomicrons within the lymph. Triglycerides are then hydrolyzed by the enzyme LPL (lipoprotein lipase) supplying peripheral tissues with free fatty acids and monoacylglycerol generating chylomicron remnants that enter the blood circulation. Chylomicron remnants are then taken up by the liver via the interaction of apolipoproteins on the chylomicron remnant surface and LDL (low density lipoprotein receptor) and LRP (low density lipoprotein receptor-related protein) receptors on hepatic endothelial cells (reviewed in Cooper, 1997). The liver is the central organ involved in adjusting the serum cholesterol level and the supply of lipids to peripheral organs via the blood circulation. Cholesterol is also secreted from the liver via bile. In the blood, lipids including cholesterol are transported via VLDL (very low density lipoprotein) and LDL (low density lipoprotein) particles to peripheral organs or back to the liver via HDL (high density lipoprotein) particles. Uptake of cholesterol from lipoprotein particles by the target organ is mediated by a specific set of apolipoproteins located on the surface of each lipoprotein particle and their interaction with receptors of the LDLR family and scavenger receptors SRB1 (scavenger receptor class B member 1) and CD36 (reviewed in Li et al., 2001; Zani et al., 2015). The composition of apolipoproteins is specific to each type of lipoprotein particle while single apolipoproteins can be present either on some lipoprotein particles or on several. Apolipoprotein B48 (ApoB48) for example is located mainly on chylomicrons and chylomicron remnants while apolipoprotein $E$ (ApoE) is present on chylomicrons, chylomicron remnants, VLDL and HDL particles (reviewed in Dominiczak and Caslake, 2011). 


\subsection{Cholesterol metabolism in the brain}

In the healthy brain, the blood-brain barrier (BBB) prevents entry of peripheral cholesterol from the circulation and brain resident cells synthesize cholesterol locally (Björkhem and Meaney, 2004). However, upon BBB impairment in pericyte deficient mice increased cholesterol influx in the brain was measured accompanied by dysregulation of cholesterol synthesis and excretion (Saeed et al., 2014). Furthermore, disturbance of BBB integrity during a variety of neurodegenerative diseases could allow access of peripheral cholesterol to the central nervous system (CNS) as demonstrated in mouse models of Pelizaeus-Merzbacher disease and multiple sclerosis (Saher et al., 2012; Berghoff et al., 2017b).

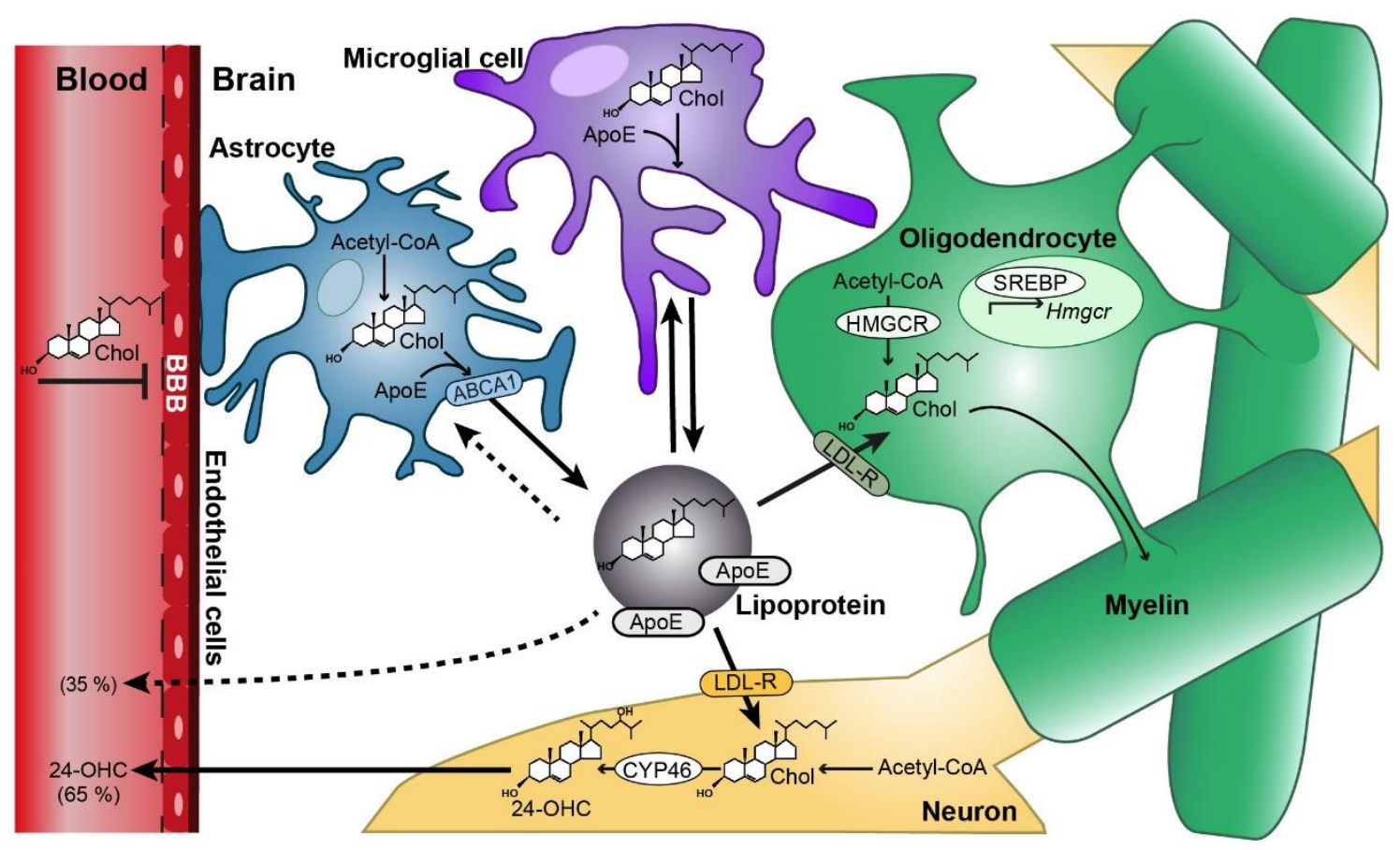

Figure 1: Cholesterol metabolism in the brain. All cells of the brain are able to synthesize cholesterol themselves. Detailed description in the text. BBB, blood-brain barrier, Chol, cholesterol, 24-OHC, 24(S)-hydroxycholesterol, ApoE, apolipoprotein E, ABCA1, ATPbinding cassette subfamily A member 1, CYP46, cholesterol 24-hydroxylase, LDL-R, lowdensity lipoprotein receptor, HMGCR, 3-hydroxy-3-methyl-glutaryl-CoA reductase, SREBP, sterol regulatory element-binding protein, PLP. Modified from Saher \& Stumpf, 2015.

All cell types of the brain are capable of cell autonomous cholesterol biosynthesis (Figure 1). Cholesterol synthesis is a complex process that is divided into two parts (reviewed in Saher et al., 2011). In the first part, the isoprenoid biosynthesis pathway (also called mevalonate pathway), acetyl-CoA is converted to farnesylpyrophosphate by a series of enzymatic reactions including the rate-limiting 
conversion of HMG-CoA to mevalonate by HMGCR (3-hydroxy-3-methyl-glutarylCoA reductase). In the second part, the sterol biosynthesis pathway, squalene synthase (SQS, encoded by the Fdft1 gene) catalyzes the first committed step in cholesterol synthesis by conversion of farnesyl-pyrophosphate to squalene. Cholesterol is finally generated via two different pathways from lanosterol with the conversion of desmosterol to cholesterol by DHCR24 (24-dehydrocholesterol reductase) being the final step in the so-called Bloch pathway and the conversion of 7-dehydrocholesterol to cholesterol by DHCR7 (7-dehydrocholesterol reductase) in the so-called Kandutsch-Russel pathway. Cellular cholesterol homeostasis is tightly regulated. Cholesterol concentration is measured in the endoplasmic reticulum (ER) by SCAP (SREBP cleavage-activating protein) complexed with SREBP (sterol regulatory element-binding protein) transcription factors (reviewed in Eberlé et al., 2004). When cellular cholesterol concentration is low SCAP-mediated SREBP translocation to the nucleus induces expression of genes involved in lipid synthesis including Hmgcr (Figure 1). While SREBP transcription factors exert partially overlapping functions in lipogenesis (reviewed in Eberlé et al., 2004), SREBP2 (encoded by the Srebf2 gene) seems to be more specific to cholesterol synthesis (Horton et al., 1998). In contrast, LXR (liver-X receptor) transcription factors are involved in the reduction of cellular cholesterol content. LXR-mediated transcriptional regulation is induced by cholesterol and oxysterols that are generated upon cellular cholesterol overload (reviewed in Kang and Rivest, 2012). Activation of LXR leads to transcriptional downregulation of Fdft1 (Wang et al., 2008) and induces expression of Apoe as well as of ATP-binding cassette transporters Abca1 and Abcg1 (Kang and Rivest, 2012), which are critically involved in cellular cholesterol export via lipoprotein particles (reviewed in Pfrieger and Ungerer, 2011).

Cholesterol transport between different cells of the brain is mediated via lipoprotein particles that under normal conditions are likely produced by astrocytes (reviewed in Pfrieger and Ungerer, 2011) and display similar densities to HDL particles (LaDu et al., 1998). Astrocyte derived lipoprotein particles contain ApoE and ApoJ (encoded by the Clu gene) apolipoproteins in vitro (Pasinetti et al., 1994; LaDu et al., 1998; Fagan et al., 1999; DeMattos et al., 2001). Uptake of cholesterol from lipoprotein particles is mediated by members of the LDLR family (Figure 1). Cholesterol is not degraded within the brain but excreted in the form of oxysterols that can readily pass the BBB. The most prominent oxysterol in the brain is $24(\mathrm{~S})$ - 
hydroxycholesterol that is generated by the enzyme cholesterol 24-hydroxylase (encoded by the Cyp46a1 gene) predominantly expressed by neurons (Dietschy and Turley, 2004). However, excretion of glia derived cholesterol likely involves a different pathway (Xie et al., 2003) via ApoE lipoprotein particles (Figure 1). The enzymes cholesterol 25-hydroxylase (encoded by the Ch25h gene) and sterol 27hydroxylase (encoded by the Cyp27a1 gene) generating 25-hydroxycholesterol and 27-hydroxycholesterol, respectively are less prominent in brain (reviewed in Russell, 2000) while expression of Ch25h was found in acutely isolated microglia (Ceglia et al., 2015).

\subsubsection{Contribution of different cell types to brain cholesterol homeostasis}

How different cell types contribute to cholesterol homeostasis in the brain is difficult to assess since all cell types of the brain are capable of cell autonomous cholesterol synthesis and can also benefit from horizontal cholesterol transfer via HDL-like lipoprotein particles (see above). It is plausible that cellular cholesterol demand is high at times of membrane expansion and cell differentiation during development. The current understanding is that the cell type predominantly producing cholesterol switches from neurons during embryogenesis to oligodendrocytes during postnatal myelination and to astrocytes in the adult brain (Pfrieger and Ungerer, 2011; Saher and Stumpf, 2015). However, to which extent different cell types contribute to cholesterol homeostasis in the adult brain in vivo is incompletely understood. Turnover of bulk cholesterol in the adult rodent brain is low with an estimated halflife of several months (Sérougne-Gautheron and Chevallier, 1973; Björkhem et al., 1997; Dietschy and Turley, 2001). However, the vast majority ( 70-80\%) of brain cholesterol in adult animals resides in myelin with low turnover (Smith, 1968; Ando et al., 2003) and high cholesterol turnover was suggested for individual neurons and astrocytes (Dietschy and Turley, 2001; Pfrieger and Ungerer, 2011). Thus, genetic tools are required to analyze cholesterol metabolism cell type specifically.

The use of SQS-flox mice (Saher et al., 2005) allows genetic inactivation of squalene synthase by targeting the Fdft1 gene in different cell types using specific Cre driver lines. Ablation of SQS function in all neurons and glial cells was lethal in newborn mice (Saito et al., 2009) highlighting the importance of cholesterol 
synthesis in the CNS. Inactivation of SQS in myelinating glia resulted in severe dysmyelination leading to ataxia and tremor in mutant mice (Saher et al., 2005). Increased levels of ApoE and LRP indicated compensatory cholesterol transfer by surrounding wild type cells mediating oligodendrocyte survival and generation of myelin (Saher et al., 2005). These findings underscore the importance of endogenous cholesterol synthesis in oligodendrocytes during myelination.

\subsubsection{Neuronal cholesterol synthesis}

Cholesterol secreted in ApoE-containing lipoprotein particles by astrocytes has been shown to promote synaptogenesis in cultured neurons (Mauch et al., 2001). Together with evidence largely based on in vitro studies this finding has promoted the notion that neurons rather rely on astrocyte derived cholesterol than synthesize cholesterol themselves (Pfrieger, 2003). This hypothesis was supported by the finding that cultured neurons produced cholesterol less efficiently than astrocytes (Nieweg et al., 2009). Do neurons require cell autonomous cholesterol synthesis in vivo? In adult mice SQS was not required for survival and function of cerebellar granule cells (Fünfschilling et al., 2007). Similarly, upon postnatal inactivation of SQS in forebrain projection neurons mutant mice were healthy and lacked any signs of brain pathology (Fünfschilling et al., 2012). Increased levels of SQS protein in astrocytes as well as upregulation of ApoE indicated that survival and functional preservation of mutant neurons was supported by astrocyte derived cholesterol (Fünfschilling et al., 2012). However, ablation of SQS in projection neurons during development resulted in layer-specific neuronal death and reduction of cortical projections despite evident microglial support including upregulation of ApoE expression (Fünfschilling et al., 2012). These findings show that neurons require cell autonomous cholesterol synthesis during development but can rely on glial cholesterol support in the adult. 


\subsubsection{Role of astrocytes in cholesterol metabolism}

Astrocytes are generated from neural precursor cells postnatally after neurogenesis is largely complete (Vallejo, 2009). Throughout development and also in the adult brain astrocytes are critically involved in establishment and maintenance of brain function. Roles of astrocytes have been identified in neuronal maturation and synaptogenesis, establishment and maintenance of the BBB, ion buffering and recycling of neurotransmitters as well as neuroinflammation and responses to injury (reviewed in Wang and Bordey, 2008). Furthermore, astrocytes support neurons with cholesterol in vitro and in vivo (see above). The extent of astrocyte involvement in brain cholesterol metabolism in vivo however is largely unknown. Genetic inactivation of the sterol sensor SCAP in GFAP (glial fibrillary acidic protein) expressing astrocytes resulted in severe brain defects including microcephaly and reduction of cholesterol and fatty acid synthesis leading to premature death (Camargo et al., 2012). These mice also exhibited defects in the maturation of presynaptic terminals resulting in reduced short- and long-term synaptic plasticity (van Deijk et al., 2017). These studies show that astrocyte derived lipids including cholesterol are crucial in brain development. Genetic deletion of SCAP however likely affects transcription of a variety of genes regulated by SREBP (Matsuda et al., 2001; Rome et al., 2008) and therefore a different approach is required focusing on cholesterol synthesis.

Understanding of the role of astrocytic cholesterol synthesis in the adult brain is limited by the lack of genetic tools that allow specific and efficient targeting of these cells. In order to study astrocyte function at desired time points several transgenic lines have been developed that express tamoxifen inducible Cre recombinase under control of promoters active in astrocytes (Ganat et al., 2006; Hirrlinger et al., 2006; Mori et al., 2006; Chow et al., 2008). Although these mouse lines are useful tools to target astrocytes in many brain regions (e.g. corpus callosum or cerebellum) only subsets of astrocytes are targeted in other regions, especially the cortex and spinal cord. The Aldh1/1 (aldehyde dehydrogenase 1 family member L1) gene is expressed in a subset of radial glia during embryonic development (Anthony and Heintz, 2007) and neuronal precursors (Foo and Dougherty, 2013) and has been identified as a pan astrocyte marker in postnatal brain (Cahoy et al., 2008). Aldh1/1 promoter driven expression of a fluorescent protein or Cre recombinase in BAC 
transgenic mouse lines (Heintz, 2004; Yang et al., 2011) confirmed that Aldh1l1 is a specific marker for CNS astrocytes including cortical astrocytes. Recently, two BAC transgenic mouse lines expressing tamoxifen inducible Cre recombinase (CreERT2) under control of the Aldh1/1 promoter were generated. Characterization of transgene activity in one line revealed that $>90 \%$ of astrocytes in the hippocampus, striatum and cortex were targeted after tamoxifen induction in adult mice (Srinivasan et al., 2016). Characterization of the other mouse line (Winchenbach et al., 2016) is part of the present study.

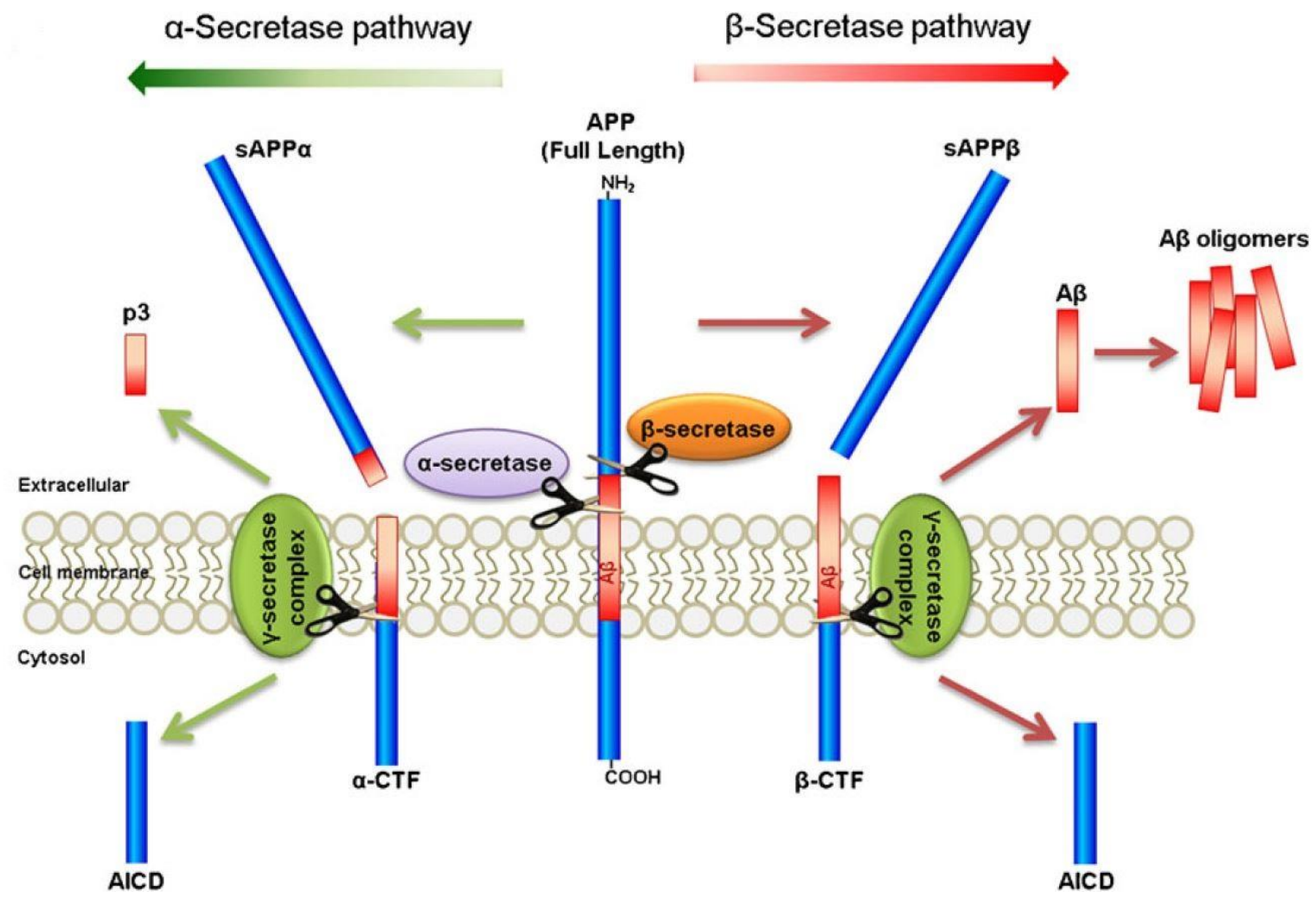

Figure 2: APP processing in the $\alpha$ - and $\beta$-secretase pathways. In the $\alpha$-secretase pathway APP is first cleaved by $\alpha$-secretase generating $\alpha-C T F$ (or C83) and SAPPa fragments followed by $\mathrm{y}$-secretase mediated shedding of $\mathrm{p3}$ and AICD fragments. In the amyloidogenic $\beta$-secretase pathway, APP is cleaved by $\beta$-secretase generating $\beta$-CTF (or C99) and SAPP $\beta$ fragments. Processing by $\gamma$-secretase then generates AICD fragments and $A \beta$ peptides that form oligomers and aggregate in extracellular deposits. APP, amyloid precursor protein, $A \beta$, amyloid- $\beta$, sAPP, soluble APP fragment, CTF, C-terminal fragment, AICD, APP intracellular C-terminal domain. Adapted from Maulik et al., 2013. 


\subsection{Alzheimer's disease}

Alzheimer's disease (AD) is the most common neurodegenerative disease worldwide and the leading cause of dementia (Reitz et al., 2011). AD patients suffer from progressive memory loss, cognitive decline and eventually loss of vital neuronal function (reviewed in Alves et al., 2012; Tarawneh and Holtzman, 2012). Histopathological hallmarks of $A D$ are extracellular deposits of amyloid- $\beta$ ( $A \beta$ ) peptides and the manifestation of intracellular neurofibrillary tangles consisting of hyperphosphorylated tau protein. The generation and deposition of misfolded $A \beta$ peptides in the brain is termed cerebral $\beta$-amyloidosis. $A \beta$ peptides are generated by sequential proteolytic processing of the amyloid precursor protein (APP) by $\beta$ secretase (BACE1) and the $\gamma$-secretase complex, whereas initial processing by $\alpha$ secretase does not lead to $A \beta$ generation (Figure 2 ). APP processing by $\gamma$-secretase generates $A \beta$ peptides of varying length, of which $A \beta 40$ is the most prominent in $A D$ brains and $A \beta 42$ likely the most toxic (reviewed in Karran et al., 2011). Pathological changes also include activation of astrocytes and microglia around $A \beta$ deposits and progressive loss of synapses as well as neuronal degeneration (reviewed in Duyckaerts et al., 2009). The cause of $A D$ is unknown for the vast majority of cases (sporadic $A D, S A D$ ) but in approximately $1-5 \%$ of affected patients, disease development is linked to inherited genetic mutations (familial AD, FAD). These mutations have been identified in the gene encoding APP (Goate et al., 1991) or in the genes encoding PSEN1 (presenilin-1, Sherrington et al., 1995) and PSEN2 (presenilin-2, Levy-Lahad et al., 1995; Rogaev et al., 1995), proteins that form the catalytic site of $\mathrm{y}$-secretase. These and several other mutations identified over the years have been shown to enhance the production of all $A \beta$ peptides or increase the $A \beta 42 / A \beta 40$ ratio in favor of the more readily aggregating $A \beta 42$ peptide (Karran et al., 2011). These findings gave rise to the amyloid cascade hypothesis (Hardy and Selkoe, 2002) which suggests the generation and deposition of $A \beta$ peptides as the driving force of $A D$ disease progression. Disease onset in FAD is 20 to 30 years earlier than in the majority of SAD cases (Karran et al., 2011) with early biomarker changes appearing even 20 to 25 years before the first symptoms (Bateman et al., 2012). 
Despite the differences in etiology, onset and disease progression between FAD and $S A D$, neuropathological changes are similar including $A \beta$ deposition and tau aggregation. Finding the cause of SAD remains an important topic of current research and includes a great variety of different approaches (De Strooper and Karran, 2016).

\subsubsection{Role of cholesterol in Alzheimer's disease}

The role of cholesterol in the pathogenesis of $A D$ is an important topic of current research and still far from understood. The importance of cholesterol in $A D$ is highlighted by the fact that 3 of the top 5 genetic risk factors for $A D$ are involved in cholesterol metabolism (AlzGene database, Bertram et al., 2007). Furthermore, age as the highest risk factor for $S A D$ also strongly affects lipid and cholesterol metabolism (Cutler et al., 2004). In AD patients clinical studies were conducted that aimed at reducing cholesterol levels by the use of statins. Results were controversial with some studies reporting beneficial effects while others did not (reviewed in Shepardson et al., 2011). Pleiotropic effects of statins likely contribute to the lack of a clear conclusion in these studies. Statins are HMGCR inhibitors and thereby not only inhibit cholesterol synthesis but affect isoprenoid synthesis in all cells. Isoprenoids are involved in post translational modification of small GTPases that regulate a variety of intracellular signaling cascades (reviewed in Wang et al., 2007). Similarly, treatment with the LXR/RXR agonist bexarotene, that modulates lipid metabolism initially showed promise in reducing $A \beta$ deposition in some (Cramer et al., 2012), but not in other mouse models of AD (Veeraraghavalu et al., 2013) and failed to reduce plaque burden in AD patients (Cummings et al., 2016). Therefore, different approaches are required to modulate cholesterol levels in the brain more directly.

Cholesterol metabolism in the brain is complex and incompletely understood (see above) which complicates research addressing the role of cholesterol in AD. Several lines of evidence derived from in vitro and preclinical studies suggest that cholesterol affects AD pathogenesis on cellular and organ level (reviewed in Maulik et al., 2013). Cholesterol enhances $A \beta$ generation in cultured neurons (Simons et al., 1998; Wahrle et al., 2002) and promotes association and endocytosis of APP 
and BACE1 in vitro (Schneider et al., 2008; Marquer et al., 2011). In early endosomes, APP is then cleaved by BACE1 generating the APP C-terminal fragment $\mathrm{C} 99$ (or $\beta$-CTF) which associates directly with cholesterol facilitating its localization to lipid rafts (Barrett et al., 2012). After recycling of APP/C99 from endosomes to the trans-Golgi network, $\gamma$-secretase processing of C99 generates A $\beta$ peptides (Wahrle et al., 2002; Choy et al., 2012). A $\beta$ peptides also directly bind cholesterol which promotes the generation of neurotoxic $A \beta$ oligomers at the plasma membrane (reviewed in Di Scala et al., 2014).

In addition to the role of cholesterol in $A \beta$ generation, cumulating evidence also implicates cholesterol transport via ApoE lipoprotein particles in the deposition of $A \beta$. Research in this field has been promoted by the finding that homozygosity of the ApoE4 isoform significantly increases AD susceptibility making ApoE4 the top genetic risk factor for AD today (AlzGene database, Bertram et al., 2007). In animal models of $A D$, deletion of the Apoe gene resulted in strongly delayed deposition of A $\beta$ without altering APP protein levels (Bales et al., 1997; Holtzman et al., 2000). Plaque formation was further delayed by additional introduction of the human Apoe gene in an isoform dependent manner with ApoE4 showing the least beneficial effect (reviewed in Tai et al., 2011). How ApoE influences $A \beta$ deposition is incompletely understood. In vitro studies have shown that $A p o E$ binds $A \beta$ and affects its oligomerization and fibrillation (reviewed in Holtzman et al., 2012). In vivo, ApoE is primarily produced by astrocytes (reviewed in Vance and Hayashi, 2010) followed by import of lipids including cholesterol into ApoE lipoprotein particles (lipidation) via ABCA1 transporters (see above). Deletion of the Abca1 gene in AD mice decreased ApoE lipidation (Hirsch-Reinshagen et al., 2004) and increased $A \beta$ deposition (Koldamova et al., 2005; Wahrle et al., 2005) while overexpression of Abca1 had the opposite effect (Wahrle et al., 2008). Genetic inactivation of the lipoprotein receptor LDLR resulted in increased ApoE levels in the brain (Cao et al., 2005; Fryer et al., 2005) and had either no effect on $A \beta$ deposition (Fryer et al., 2005) or increased A load in AD mouse models (Cao et al., 2005). Together these studies show that lipidation status and extent of ApoE-mediated lipid transport within the brain affect $A \beta$ deposition. However, the underlying mechanisms are not well understood. Next to directly affecting distribution and subsequent deposition of $A \beta$, transport of $A \beta$ associated with ApoE lipoprotein particles could also affect the clearance of these peptides by different cell types or their excretion from the brain 
(Holtzman et al., 2012). Proteolytic degradation of $A \beta$ is complex and can involve a variety of enzymes that are present in neurons and glial cells as well as in the extracellular space (reviewed in Saido and Leissring, 2012). The endopeptidase neprilysin (encoded by the Mme gene) is expressed in neurons and involved in $A \beta$ degradation in axons and synapses (Fukami et al., 2002). Insulin-degrading enzyme (encoded by the Ide gene) is expressed by many cell types (Zhang et al., 2014) and is also located in the extracellular space (Saido and Leissring, 2012). In contrast, endothelin-converting enzymes ECE1 and ECE2 (encoded by Ece1 and Ece2) seem to degrade $A \beta$ primarily intracellularly (Eckman et al., 2002). ApoE likely influences the degradation of $A \beta$ by glial cells. Some studies proposed that $A p o E$ and $A \beta$ do not interact but rather compete for the receptor for endocytosis (Basak et al., 2012; Verghese et al., 2013), while another study proposed that lipidated $A p o E$ lipoprotein particles associate with $A \beta$ and mediate degradation either by pinocytosis or extracellular proteolytic cleavage (Jiang et al., 2008). Clearance of $A \beta$ from the brain via the BBB might also be linked to cholesterol metabolism (Zhao et al., 2015). Excretion of cholesterol from the brain is mediated either via 24(s)hydroxycholesterol or ApoE (see above). Loss of Cyp46a1 did not affect $A \beta$ deposition but increased life expectancy in an $A D$ mouse model (Halford and Russell, 2009). Furthermore, $A \beta$ binds to receptors of the LDLR family at the BBB (Zhao et al., 2015) which also mediate cholesterol transport within the brain (see above). Excretion of $A \beta$ to the blood circulation is mediated by LRP1 (Storck et al., 2016) and LRP2 (Bell et al., 2006). Overexpression of Ldlr decreased brain ApoE levels and increased efflux of $A \beta$ at the BBB by an unknown mechanism (Castellano et al., 2012). In summary, several lines of evidence indicate a role of cholesterol in the generation, distribution and clearance of $A \beta$ in the brain. However, the underlying mechanisms are not well understood and the contribution of different cell types to these processes in vivo are unknown. 


\subsection{Aims of the study}

The contribution of astrocytes to brain cholesterol metabolism in vivo is not well understood due to the lack of genetic tools that allow efficient and specific targeting of adult astrocytes. Therefore, the first aim of this study was to characterize the newly generated Aldh111-CreERT2 mouse line (Winchenbach et al., 2016) regarding efficiency and specificity of Cre recombination in adult astrocytes. A direct approach to inactivate cholesterol synthesis in adult astrocytes has so far been lacking. To address this, in the second part of this project conditional mutant mice were generated that lack expression of SQS in astrocytes. Conditional mutants were analyzed with respect to astrocyte survival and viability. Astrocytes are tightly associated with microvessels in the brain (Mathiisen et al., 2010) and involved in regulation of BBB integrity via ApoE (Bell et al., 2012). Therefore, we assessed whether loss of cholesterol synthesis in astrocytes affects BBB integrity in conditional mutants.

Evidence largely based on in vitro studies implicate cholesterol metabolism in the generation and deposition of $A \beta$ in Alzheimer's disease (Maulik et al., 2013). Astrocytes supply neurons with cholesterol via ApoE lipoprotein particles in vitro (Mauch et al., 2001). In vivo, SQS deficient neurons rely on cholesterol support by other cells, likely astrocytes, to maintain cellular cholesterol content and function (Fünfschilling et al., 2012). Reduction in neuronal cholesterol content inhibits the generation of $A \beta$ in vitro (Simons et al., 1998; Wahrle et al., 2002) and direct interaction of APP and cholesterol could enhance $\beta$-secretase mediated $A \beta$ production in cholesterol-rich lipid rafts (Barrett et al., 2012). Therefore, loss of cholesterol support by astrocytes could (I) influence neuronal cholesterol content and thereby the generation of $A \beta$. To address this, SQS was inactivated in astrocytes of 5xFAD mice, an animal model of AD (Oakley et al., 2006). In these compound mutants, APP processing and $A \beta$ deposition was assessed.

Lipid transport within the brain likely influences the distribution and deposition of $A \beta$ (Holtzman et al., 2012). Decreased ApoE lipidation (Hirsch-Reinshagen et al., 2004) resulted in increased $A \beta$ deposition in mice lacking Abca1 (Koldamova et al., 2005; Wahrle et al., 2005) while overexpression of Abca1 resulted in decreased $A \beta$ deposition (Wahrle et al., 2008). Therefore, loss of cholesterol synthesis in 
astrocytes could (II) modulate lipid transport via $A p o E$ lipoprotein particles and thereby affect $A \beta$ distribution and deposition. Furthermore, ApoE promotes the degradation of $A \beta$ by astrocytes and microglia in vitro (Koistinaho et al., 2004; Jiang et al., 2008). Promoting lipidation of $A p o E$ increased $A \beta$ clearance by microglia in vitro and reduced $A \beta$ deposition in vivo (Jiang et al., 2008). Thus, loss of SQS in astrocytes could (III) affect clearance and degradation of $A \beta$ by astrocytes and microglia. To address these hypotheses (II, III), cholesterol homeostasis and astrocyte and microglia responses were analyzed.

In a complimentary approach SQS was inactivated in forebrain projection neurons of 5xFAD mice to assess the role of neuronal cholesterol synthesis in cerebral $\beta$ amyloidosis. As reported in vitro (Simons et al., 1998; Wahrle et al., 2002), loss of neuronal cholesterol synthesis could (IV) directly influence APP processing and A $\beta$ generation in these mice. 


\section{Results}

The majority of results (including the text) presented in section 2.1 and 2.2 are adapted from our manuscript published in 2016 (Winchenbach et al., 2016).

\subsection{Are astrocytes efficiently targeted in Aldh1l1-CreERT2 transgenic mice?}

Cre-mediated recombination of target genes in adult astrocytes requires the use of an inducible expression system, because many promoters of the astrocyte lineage are also active in multipotent neural stem cells in the subventricular and subgranular zones (Christie et al., 2013). Thus, transgenic mouse lines have been generated for tamoxifen-inducible Cre recombination of target genes in mature astrocytes (Ganat et al., 2006; Hirrlinger et al., 2006; Mori et al., 2006; Slezak et al., 2007; Chow et al., 2008). However, none of them achieves sufficient recombination to study the function of genes in the majority of cortical and spinal cord astrocytes. Aldh1l1 has been identified as a highly specific marker for astrocytes in the brain (Cahoy et al., 2008), which was verified in BAC transgenic mice with a fluorescent reporter protein or constitutive Cre expression under control of the Aldh1l1 promoter (Heintz, 2004; Yang et al., 2011). Therefore, our group has generated Aldh1l1-CreERT2 BAC transgenic mice expressing tamoxifen-inducible Cre recombinase under control of the Aldh1l1 promoter (Winchenbach et al., 2016) using the cloning strategy shown in Figure $3 \mathrm{~A}$. In order to assess Cre recombination, mice carrying the Aldh111CreERT2 transgene (Figure $3 \mathrm{~B}$ ) were crossed with ROSA26-tdTomato reporter mice (Madisen et al., 2010), termed astro-tdTo mice, or with ROSA26-Eyfp reporter mice (Srinivas et al., 2001). First, we determined the leakiness of reporter expression in adult Aldh111-CreERT2 mice. After corn oil injections in astro-tdTo mice, we found very few labeled cells (less than 5 per section), demonstrating that the inducible Cre system operates tightly. In parallel experiments, adult astro-tdTo mice were analyzed 7 days after tamoxifen induction. Sagittal brain sections revealed numerous tdTomato Cre reporter expressing cells, which in the forebrain exhibited the typical morphology of protoplasmic astrocytes (Figure 3). Co-labeling revealed that almost all S100beta (S100 calcium-binding protein B) positive cells in hippocampus and cerebral cortex expressed tdTomato (Figure 3, Table 1). 
A BAC containing the Aldh1/1 allele
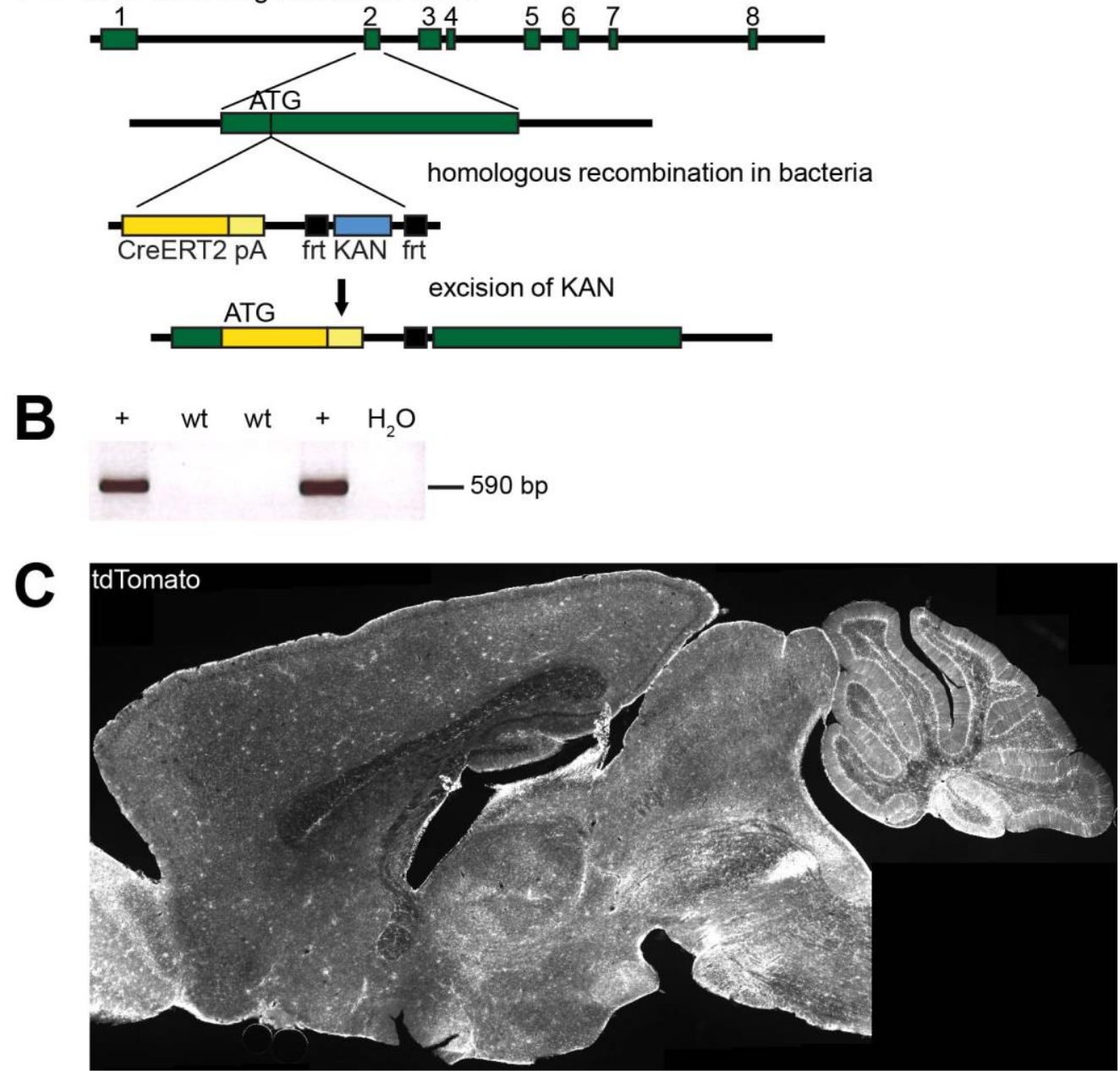

D
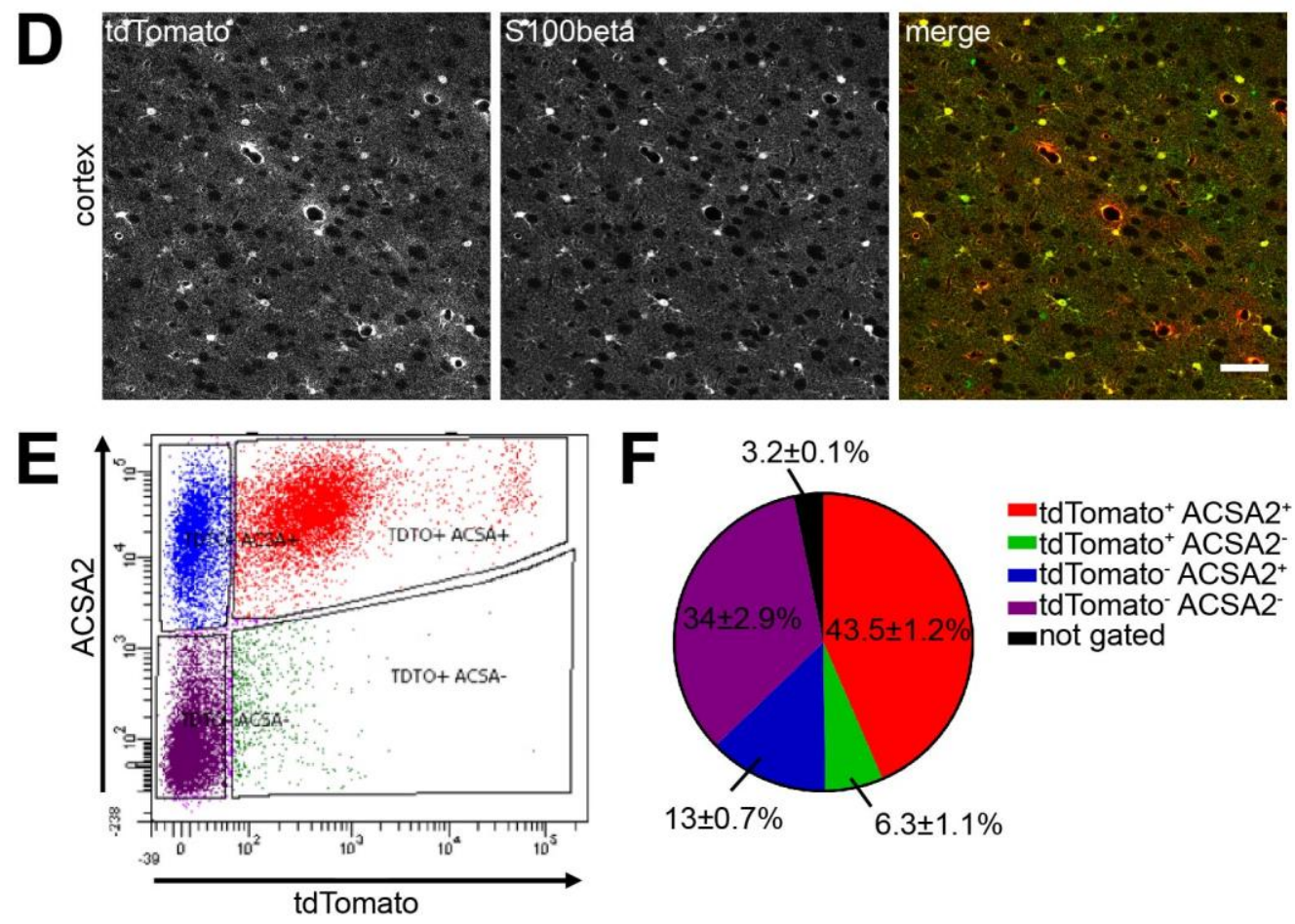
Figure 3: The Aldh1l1 BAC transgene efficiently targets CNS astrocytes. A) Scheme of the cloning strategy of the Aldh1l1-CreERT2 BAC transgene. B) Genotyping PCR from Aldh1l1-CreERT2 mice (+), wild type (wt) mice and from water (as a negative control). C) Direct fluorescence of the Cre-reporter tdTomato in sagittal brain sections of astro-tdTo mice. D) Immunolabeling of the astrocyte marker S100beta (green) in the cortex reveals almost complete overlap with the tdTomato Cre reporter (red) in astrocytes. Scale, $50 \mu \mathrm{m}$. E) Dot plot showing the gating strategy used during FACS analysis of cortical astrocytes of adult astro-tdTo mice immunolabeled with the astrocyte marker ACSA2. F) Quantification of cell populations shown in E. Values are expressed as per cent of all analyzed cells per animal ( $n=3$ animals, \pm s.e.m.). Panels $A, C$ and $D$ adapted from Winchenbach et al., 2016. Initial generation of Aldh1l1-CreERT2 mice was performed by Dr. Gesine Saher. FACS analysis was performed together with Dr. Francesca Odoardi.

Analysis of astrocytes isolated from the cortex of adult astro-tdTo animals by fluorescence-activated cell sorting (FACS) revealed that $87.5 \pm 1.8 \%(n=3, \pm$ s.e.m.) of cells labeled with the astrocyte marker ACSA2 (astrocyte cell surface antigen-2) expressed tdTomato while $77.0 \pm 0.5 \%$ of tdTomato expressing cells were ACSA2 positive (Figure $3 \mathrm{E}, \mathrm{F}$ ).

Table 1: Efficiency and specificity of Aldh1l1-CreERT2 mediated recombination in brain. Efficiency and specificity of inducible Cre mediated recombination in adult Aldh1/1CreERT2 mice crossbred with Cre reporter ROSA26-tdTomato or ROSA26-Eyfp. For each value shown (average percentage), cells were counted on eight confocal images and two sections for each of $n=3-4$ animals. Efficiency is expressed as percent Cre reporter positive cells of all S100beta labeled cells. Specificity is expressed as percentage of all Cre reporter positive cells that lack immuno-labeling for S100beta. Adapted from Winchenbach et al., 2016.

\begin{tabular}{|l|l|c|c|}
\hline \multicolumn{2}{|l|}{ Region } & \multicolumn{1}{l|}{$\begin{array}{l}\text { Co-labeled } \\
\text { cells (\%) }\end{array}$} & $\begin{array}{l}\text { Number of } \\
\text { cells }\end{array}$ \\
\hline Efficiency & \multicolumn{2}{|l|}{} \\
\hline Cortex (astrocytes) & tdTomato/S100beta & $92 \pm 2$ & 1868 \\
\cline { 2 - 4 } & EYFP/S100beta & $62 \pm 2$ & 2038 \\
\hline Cerebellum (Bergman glia) & EYFP/S100beta & $89 \pm 1$ & 1460 \\
\hline Corpus callosum & tdTomato/S100beta & $85 \pm 1$ & 713 \\
\hline Fimbria & tdTomato/S100beta & $94 \pm 2$ & 145 \\
\hline Specificity & \multicolumn{3}{|l}{} \\
\hline Cortex (astrocytes) & S100beta neg./tdTomato & $12 \pm 3$ & 1943 \\
\cline { 2 - 4 } & S100beta neg./EYFP & $19 \pm 3$ & 1553 \\
\hline Cerebellum (Bergman glia) & S100beta neg./EYFP & $6 \pm 1$ & 1397 \\
\hline Fimbria & S100beta neg./tdTomato & $4 \pm 1$ & 143 \\
\hline
\end{tabular}


For comparison, when using a less sensitive ROSA26-Eyfp Cre reporter line (Srinivas et al., 2001) in corresponding experiments, only two thirds of all S100beta positive cells in the cortex were also EYFP positive (Table 1). Thus, although both Cre reporter lines were generated as a knock-in into the endogenous ROSA26 locus, the recombination efficacy achieved is clearly different, in agreement with previous reports (Srinivas et al., 2001; Madisen et al., 2010). This finding illustrates the need to determine recombination efficiency individually for each combination of Cre allele and floxed target gene. The expression pattern of some astroglial marker proteins, such as GFAP (glial fibrillary acidic protein), differs between protoplasmic astrocytes in the cortex and fibrous astrocytes in white matter. We therefore assessed the efficacy of Cre recombination separately for the corpus callosum, fimbria, hippocampus and spinal cord. Again, in all these regions a large majority of astrocytes, as defined by S100beta or GFAP, expressed Cre reporter, e.g. $85 \pm 1 \%$ in the corpus callosum and $94 \pm 2 \%$ in the fimbria ( $n=3$ animals, Figure 4, Table 1). Co-labeling with GFAP was not used for cell counts because of the protein's low abundance in cell bodies which makes unequivocal quantification difficult. In the cerebellum, a large fraction ( $89 \pm 1 \%$ ) of S100beta positive Bergman glia cells expressed the Cre reporter EYFP (Figure $4 \mathrm{C}$, Table 1). While $3.3 \pm 0.3 \%$ of parvalbumin positive interneurons of the molecular layer expressed the tdTomato Cre reporter, none was double positive in corresponding experiments using the EYFP Cre reporter, confirming the sensitivity of the tdTomato reporter with a tendency for off-target recombination. 

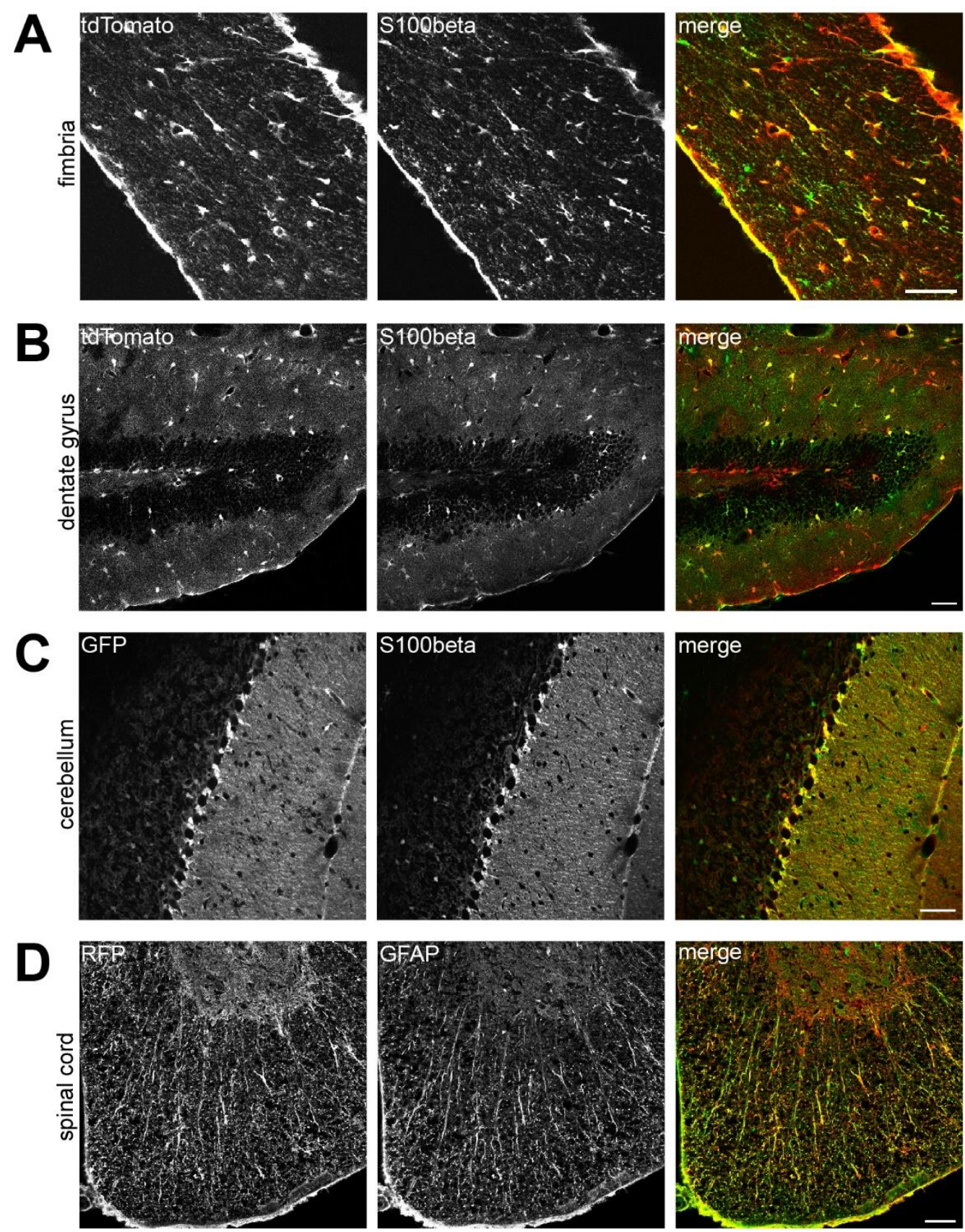

Figure 4: Inducible targeting of Bergman glia and white matter astrocytes. Coimmunolabeling of the astrocyte marker S100beta or GFAP (green) with Cre reporter (in red: direct tdTomato fluorescence, GFP anti-YFP or RFP anti-tdTomato) in fimbria (A), the dentate gyrus of the hippocampus (B), cerebellum (C) and spinal cord (D) reveals almost complete overlap of the transgene with astrocytes. Scale, $50 \mu \mathrm{m}$. Adapted from Winchenbach et al. (2016). 

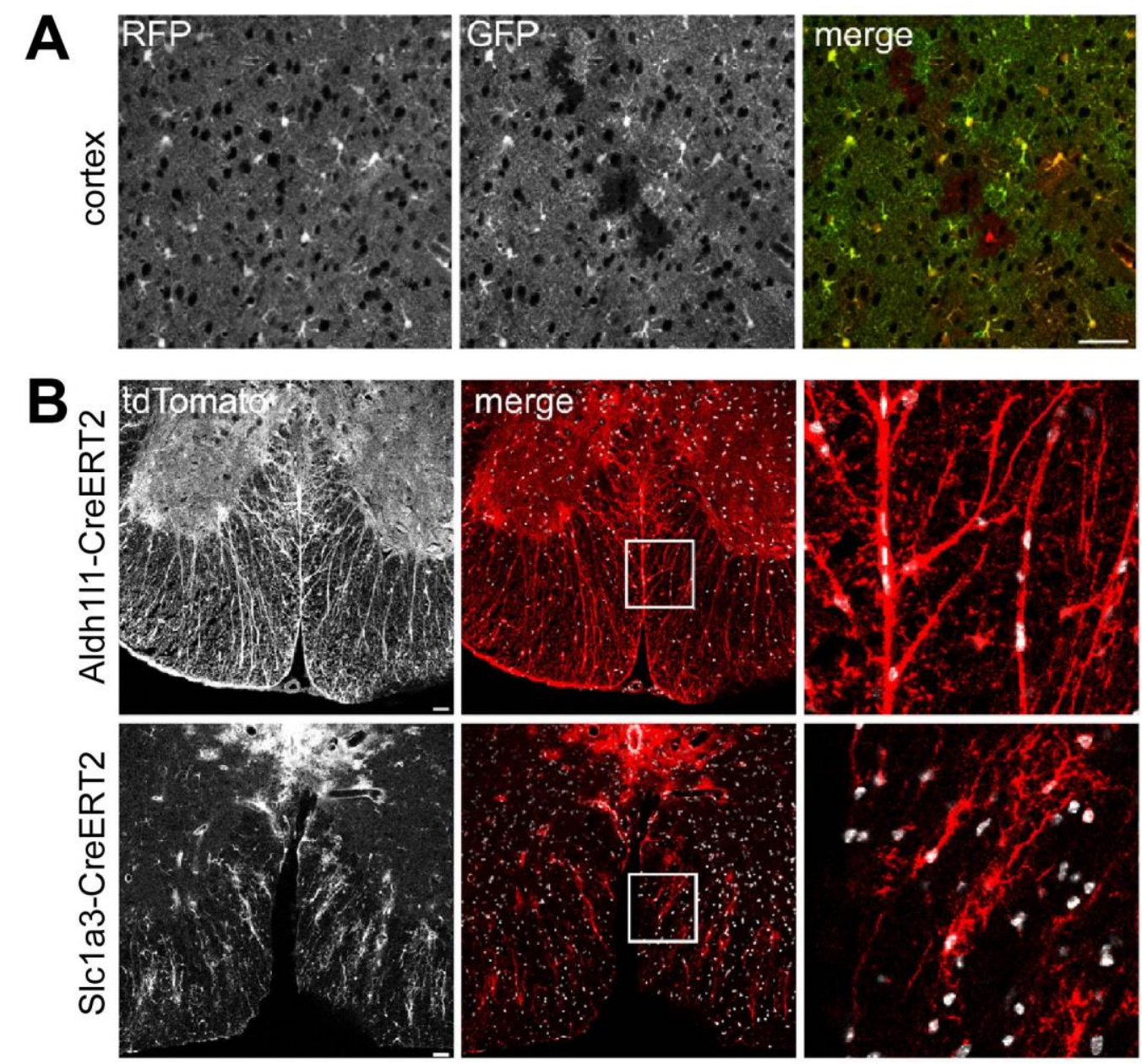

Figure 5: Comparison with other transgenes targeting astrocytes. A) Coimmunolabeling of the Cre reporter tdTomato (red, anti-RFP) and EGFP (green, anti-GFP) in triple transgenic mice (Aldh1l1-CreERT2*ROSA26-tdTo*Aldh1l1-Egfp) in cortical sections. Scale, $50 \mu \mathrm{m}$. B) Direct fluorescene of the Cre reporter tdTomato (red) and DAPI (white) in spinal cord sections of astro-tdTo and Slc1a3-CreERT2*ROSA26-tdTo transgenic animals. Scales, $50 \mu \mathrm{m}$. Adapted from Winchenbach et al. (2016).

Cre reporter expression was also observed in some neurons in the dentate gyrus and olfactory bulb, likely reflecting some recombination in adult neural stem cells in the subgranular and subventricular zone, followed by the migration of labeled progeny through the rostral migratory stream (Figure $3 \mathrm{C}$ ). Next, we compared Aldh111-CreERT2 mediated recombination with the expression pattern of EGFP in Aldh1l1-Egfp transgenic mice, generated with a similar BAC based strategy (Heintz, 2004). As expected, reporter and EGFP expression was nearly identical in the cortex, confirming the high efficiency of CreERT2 mediated induction of the tdTomato reporter (Figure $5 \mathrm{~A}$ ). Finally, in comparison with Slc1a3-CreERT2 (Mori et al., 2006), Aldh1l1-CreERT2 mediated recombination of the tdTomato reporter revealed nearly complete recombination of astrocytes in spinal cord white matter, whereas Slc1a3-CreERT2 mediated fluorescence appeared patchy (Figure 5 B). 


\subsection{What is the cellular specificity of Cre expression in Aldh1l1-CreERT2}

mice?

Next, the cell-type specificity of the Aldh1l1-CreERT2 transgene was tested. Colocalization of tdTomato with markers for neurons (NSE, neuron specific enolase) or microglia (Iba1, ionized calcium binding adaptor molecule 1) was virtually absent (Figure 6, Table 2). However, we observed a small fraction of Cre reporter positive cells co-localizing with Olig2 (oligodendrocyte lineage transcription factor 2), a transcription factor found in all oligodendrocyte lineage cells, including oligodendrocyte precursor cells (Figure 6 B).

Table 2: Specificity of Aldh1l1-CreERT2 mediated recombination in brain. Specificity of inducible Cre mediated recombination in adult astro-tdTo mice. For each value (average percentage), cells were counted on eight confocal pictures and two sections for each of $n=$ 4 animals. Specificity is expressed as percentage of cells that show Cre reporter expression of all cell type marker positive cells. Adapted from Winchenbach et al., 2016.

\begin{tabular}{|l|l|c|c|}
\hline Region & Marker & Co-labeled cells (\%) & Number of cells \\
\hline Cortex & tdTomato/NSE & 0 & 1000 \\
\cline { 2 - 4 } & tdTomato/lba1 & $0.4 \pm 0.3$ & 1157 \\
\cline { 2 - 4 } & tdTomato/CAll & $1.8 \pm 0.9$ & 443 \\
\cline { 2 - 4 } & tdTomato/EYFP (NG2) & $3.4 \pm 0.8$ & 1275 \\
\hline Cerebellum & tdTomato/Parvalbumin & $3.3 \pm 0.3$ & 2504 \\
\hline
\end{tabular}

* analyzed in triple transgenic mice (Aldh1l1-CreERT2*ROSA26-tdTomato*NG2-Eyfp) by Dr. Gesine Saher. 

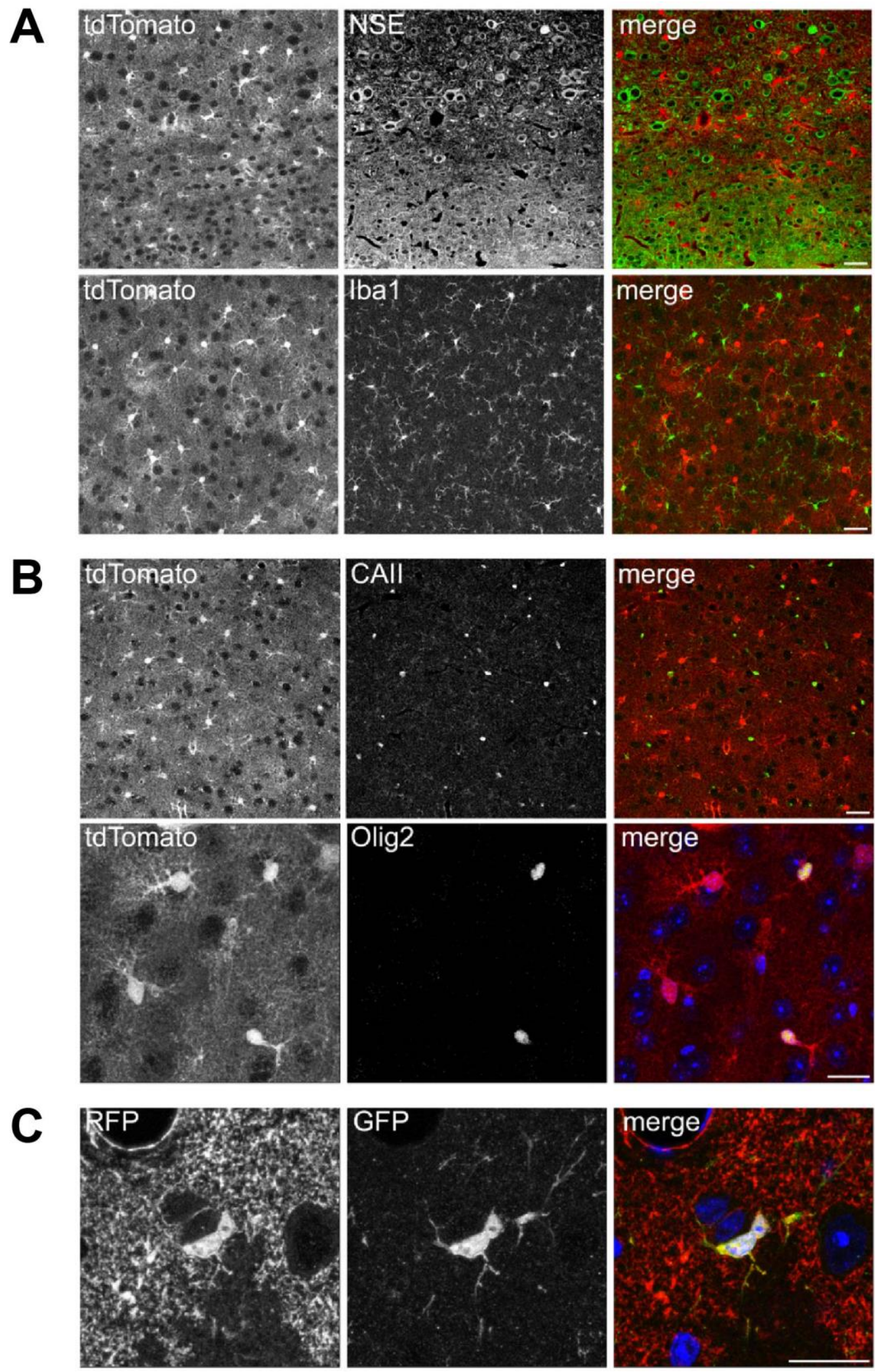
Figure 6: Specificity of Aldh1l1-CreERT2 mediated recombination. A) Direct fluorescence of the Cre-reporter tdTomato (red) and immunolabeling of neurons (green, NSE) and microglia (green, Iba1) on cortical sections. Scales, $50 \mu \mathrm{m}$. B) Direct fluorescence of the Cre reporter tdTomato (red) and immunolabeling of mature oligodendrocytes (green, CAll, scale, $50 \mu \mathrm{m}$ ) and oligodendroglia (green, Olig2, scale, $20 \mu \mathrm{m}$ ). C) Co-immunolabeling of the Cre reporter tdTomato (red, anti-RFP) and EYFP (green, anti-GFP) in triple transgenic mice (Aldh1l1-CreERT2*ROSA26-Tdto*NG2-Eyfp) revealing co-labeling in a small fraction of cells. Scale, $20 \mu \mathrm{m}$. Adapted from Winchenbach et al. (2016).

Similarly, in triple transgenic mice that additionally express EYFP under control of the endogenous NG2 (neural/glial antigen 2) promoter (Karram et al., 2008), we identified $3.4 \%$ of double labeled cells, presumably oligodendrocyte precursor cells based on their localization and morphology. However, co-localization with a marker of mature oligodendrocytes (CAll, carbonic anhydrase 2) was negligible 12 days after tamoxifen injections, and did not increase in mice that were analyzed 27 weeks after recombination (tamoxifen induction at 16 weeks of age). This suggests that the small percentage of Aldh111-CreERT2 expressing NG2 glia does not give rise to oligodendrocytes. Taken together, Aldh1l1-CreERT2 mice efficiently and specifically target the majority of astrocytes in brain and spinal cord.

\subsection{Conditional inactivation of cholesterol synthesis in astrocytes}

Astrocytes are assumed to be the major cholesterol supplier in the adult brain (Dietschy and Turley, 2001) and enhanced production and transport of cholesterol by astrocytes supported neurons lacking cell autonomous cholesterol synthesis (Fünfschilling et al., 2012). However, an in vivo approach to directly assess the role of cholesterol synthesis specifically in adult astrocytes has been lacking. In order to address this question we crossed Aldh111-CreERT2 mice (Winchenbach et al., 2016) harboring the Cre reporter tdTomato (Madisen et al., 2010) with SQS-flox mice (Saher et al., 2005). In SQS-flox mice exon 5 of the gene encoding squalene synthase (Fdft1), the enzyme catalyzing the first committed step in cholesterol biosynthesis, is flanked by loxP sites allowing inactivation of the gene upon Cremediated excision of exon 5. Offspring of these animals with the genotype Aldh111CreERT2*ROSA26+/tdTomato*Fdft $1^{\text {floxflox }}$, referred to as astroSQS-tdTo mice, were injected with tamoxifen at 8 weeks of age to induce recombination in astrocytes 
(Figure 7 A). Conditional mutants progressively lost weight and had to be sacrificed 17 to 18 days after tamoxifen induction. To assess whether this was due to a disturbed cholesterol homeostasis arising from Aldh111-CreERT2 transgene activity in peripheral organs such as liver (Winchenbach et al., 2016), serum cholesterol levels were measured at day 16 after tamoxifen injection. Indeed, total serum cholesterol concentration in these animals was reduced to $38.7 \pm 7.6 \%(n=3, \pm$ s.e.m.) compared to untreated wild type animals (Figure $7 \mathrm{C}$ ). In order to restore peripheral cholesterol levels mice received chow supplemented with 0.5\% cholesterol ("chol chow", Figure 7 A) which rescued weight loss and increased serum cholesterol levels (Figure $7 \mathrm{~B}, \mathrm{C}$ ). AstroSQS-tdTo animals on cholesterol chow appeared healthy and did not show any obvious behavioral abnormalities.
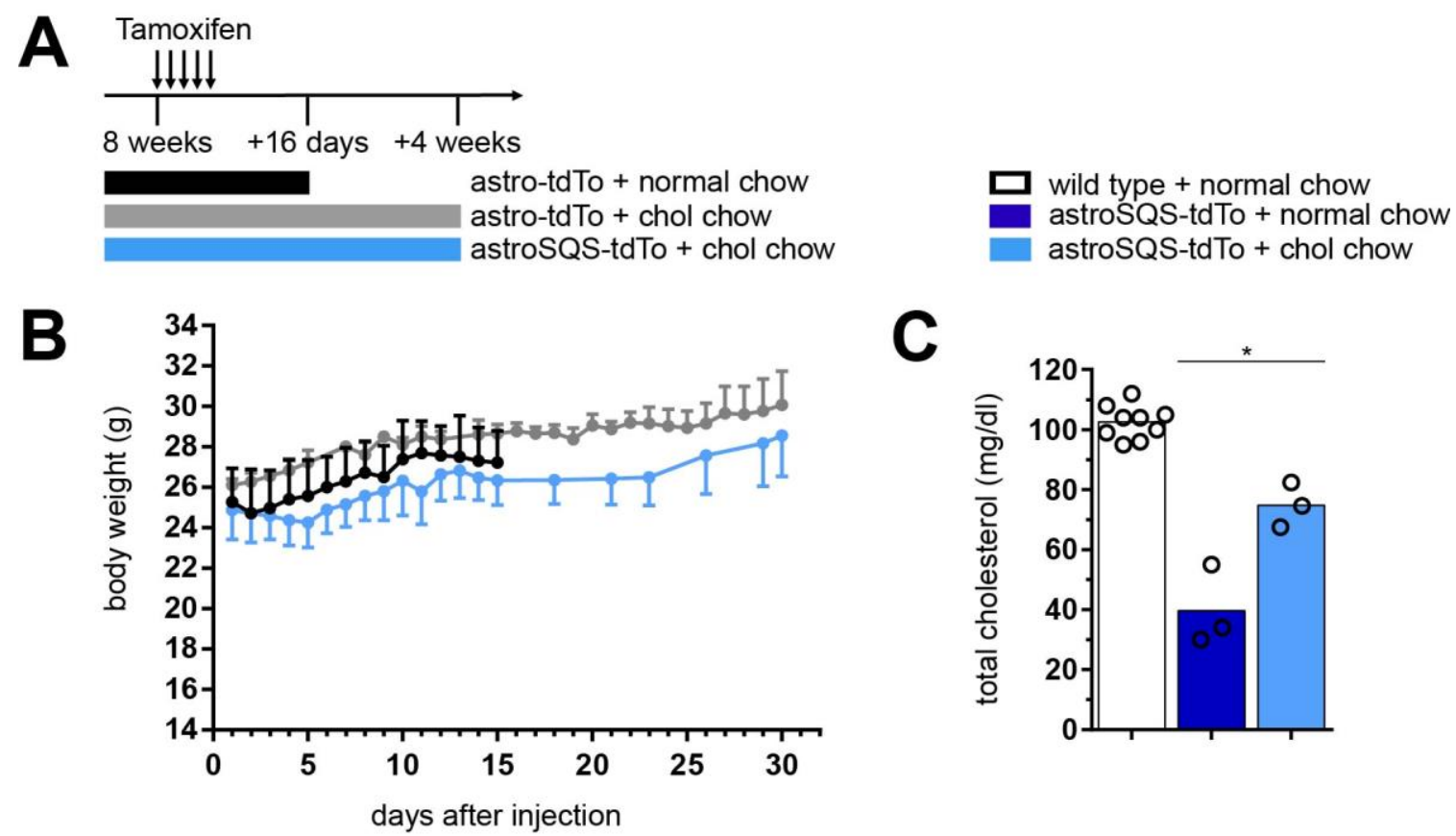

Figure 7: Weight loss rescued by cholesterol diet. A) Scheme depicting the time course of tamoxifen injections and cholesterol treatment until analysis of the animals 16 days or 4 weeks after the first tamoxifen injection. Animals received either normal chow or normal chow supplemented with $0.5 \%$ cholesterol $(\mathrm{w} / \mathrm{w})$ throughout the experiment starting 3 days before the first tamoxifen injection. B) Body weight of male animals assessed from the first day of tamoxifen injection until sacrifice of the animals. Data is expressed as mean weight \pm s.d. of $n=3-9$ animals. C) Total serum cholesterol concentration of male astroSQS-tdTo animals receiving normal chow or cholesterol supplemented chow ( $n=3$ animals each) analyzed on day 16 after the first tamoxifen injection compared to untreated male wild type animals $(n=9)$. Bars represent mean values with individual data points. Asterisks represent significant differences with ${ }^{*} p<0.05$ (unpaired Student's t-test). 


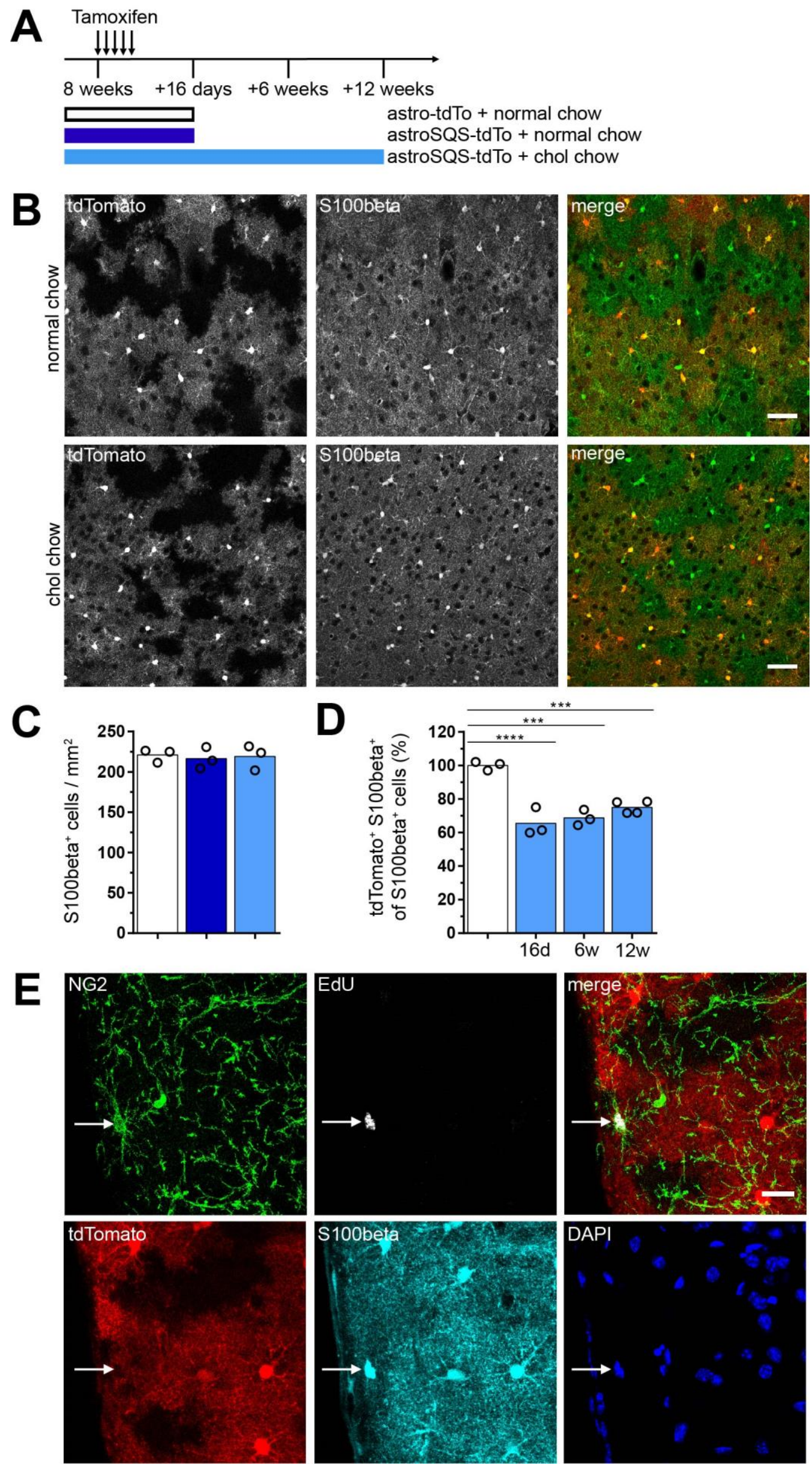


Figure 8: Loss of tdTomato signal in conditional mutants is not accompanied by astrocyte loss. A) Scheme depicting the time course of tamoxifen administration and duration of cholesterol chow treatment. Male and female astro-tdTo and astroSQS-tdTo animals on normal chow were analyzed 16 days after the first tamoxifen injection while astroSQS-tdTo animals receiving cholesterol supplemented chow were additionally analyzed 6 and 12 weeks after the first tamoxifen injection. B) Direct fluorescence of the Cre reporter tdTomato (red) and immunolabeling of the astrocyte marker S100beta (green) in cortical sections of astroSQS-tdTo animals receiving either normal chow (top row) or normal chow supplemented with $0.5 \%$ cholesterol (bottom row) 16 days after the first tamoxifen injection. Scales, $50 \mu \mathrm{m}$. C) Quantification of S100beta positive cells per $\mathrm{mm}^{2}$ in cortical layers II to IV in astro-tdTo mice on normal chow and astroSQS-tdTo animals receiving either normal or cholesterol chow at day 16 after the first tamoxifen injection. Cells were counted on 4 confocal pictures per animal. Bars represent mean values of $n=3$ animals per group with individual data points. D) Quantification of S100beta immunoreactive cells that also express the Cre reporter tdTomato in cortical sections of astro-tdTo animals on normal chow ( +16 days) and astroSQS-tdTo animals on cholesterol chow $(+16$ days,+6 and +12 weeks). Cells were counted on 4 confocal pictures per animal. Data are expressed as per cent of all S100beta immunoreactive cells per animal normalized to astro-tdTo animals $(100 \%)$. Bars represent mean values with individual data points $(n=3-4$ animals per group). E) Maximum intensity projection of immunolabeling of NG2 (green), S100beta (cyan), direct tdTomato fluorescence (red) and labeling of EdU (white) and DAPI (blue) in the cortex of astroSQS-tdTo animals on cholesterol chow 16 days after the first tamoxifen injection. Arrow indicates an EdU positive NG2 cell. Scale, $20 \mu \mathrm{m}$. Asterisks represent significant differences with ${ }^{* * *} p<0.001,{ }^{* * * *} p<0.0001$ (one-way ANOVA with Tukey's multiple comparison test).

\subsubsection{Do astrocytes survive that lack cell autonomous cholesterol synthesis?}

In order to answer the question whether mutant astrocytes survive, sagittal brain sections of astroSQS-tdTo mice receiving normal chow were immunolabeled for the astrocyte marker S100beta. Immunolabeled cells in the cortex exhibited the typical bushy morphology of protoplasmic astrocytes and were evenly distributed (Figure 8 B, upper panel). Quantification revealed no difference in the density of S100beta positive astrocytes in the cortex of astroSQS-tdTo animals on normal chow compared to astro-tdTo controls (Figure $8 \mathrm{C}$ ). There was also no difference in morphology (Figure $8 \mathrm{~B}$, lower panel) and density (Figure $8 \mathrm{C}$ ) of S100beta positive astrocytes in the cortex of astroSQS-tdTo animals on cholesterol chow compared to animals on normal chow or astro-tdTo controls. Furthermore, immunolabeling for Iba1 revealed that microglia exhibited a ramified morphology, equal distribution (Figure $9 \mathrm{~A}$ ) and unchanged density (Figure $9 \mathrm{~B}$ ) in the cortex of astroSQS-tdTo animals indicating absence of microglia activation. Together, these data suggest that astrocytes do not undergo apoptosis in astroSQS-tdTo animals. 

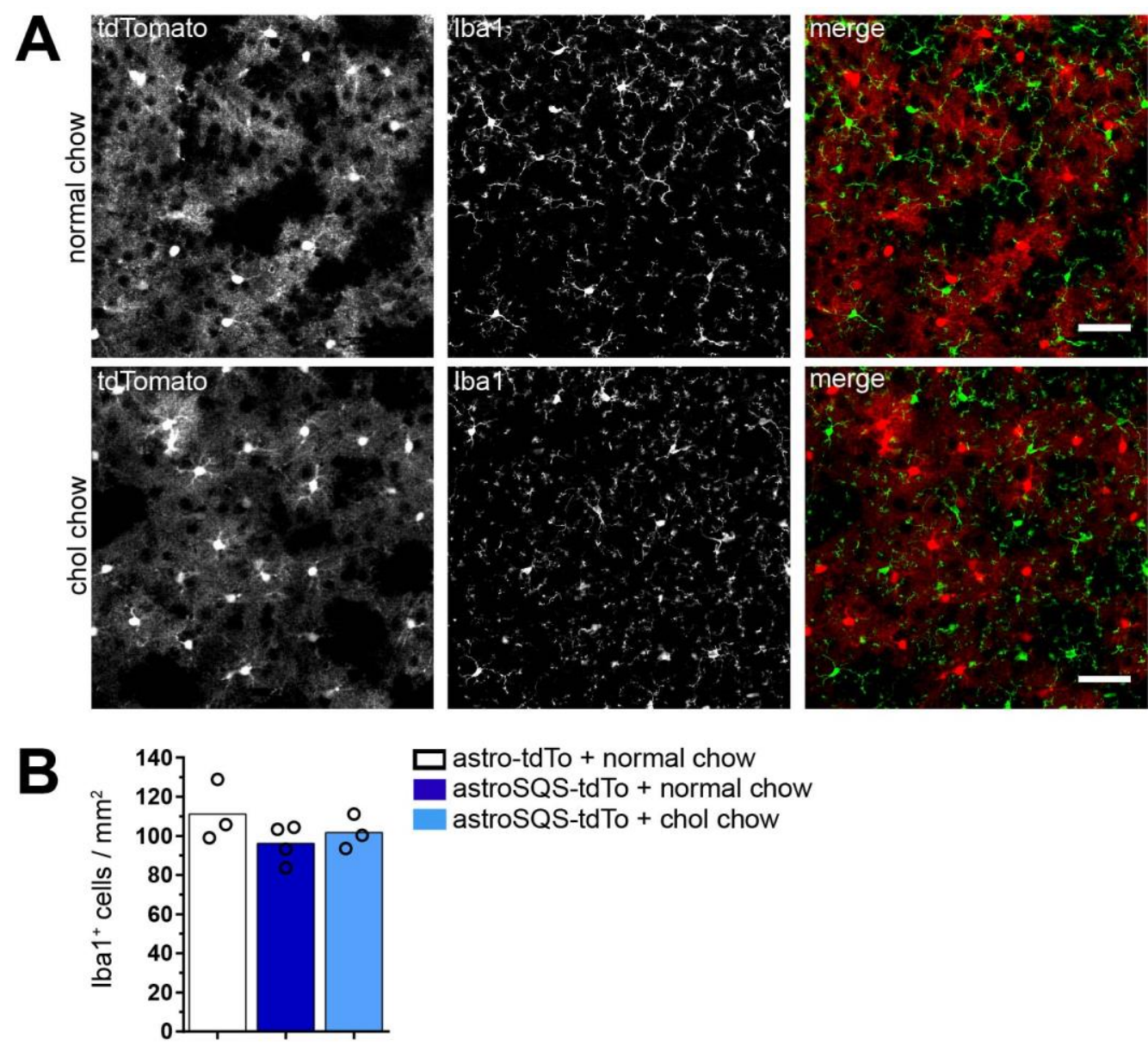

astro-tdTo + normal chow astroSQS-tdTo + normal chow astroSQS-tdTo + chol chow

Figure 9: Absence of microglia activation in conditional mutants. A) Direct fluorescence of the Cre reporter tdTomato (red) and immunolabeling of the microglia marker Iba1 (green) in cortical sections of astroSQS-tdTo animals receiving either normal chow (top row) or normal chow supplemented with $0.5 \%$ cholesterol (bottom row) 16 days after the first tamoxifen injection. Scales, $50 \mu \mathrm{m}$. B) Quantification of lba1 positive cells per $\mathrm{mm}^{2}$ in cortical layers II to IV in astro-tdTo mice on normal chow and astroSQS-tdTo animals receiving either normal or cholesterol chow at day 16 after the first tamoxifen injection. Bars represent mean values of $n=3-4$ animals per group with individual data points.

\subsubsection{What is the reason for reduced tdTomato signal in astroSQS-tdTo animals?}

Surprisingly, albeit unchanged astrocyte density, the fraction of S100beta positive astrocytes also positive for the Cre reporter tdTomato was reduced to $65 \pm 5 \%$ (n $=3, \pm$ s.e.m.) in astroSQS-tdTo mice in cortex (Figure $8 \mathrm{D}$ ) and throughout the brain compared to astro-tdTo animals. This reduction remained unchanged between 16 days and 12 weeks after tamoxifen injection indicating that this population of astrocytes is stable over time (Figure $8 \mathrm{D}$ ). In order to assess whether this reduction 
in tdTomato positive cells was due to insufficient tamoxifen administration the duration of tamoxifen treatment was increased from 5 to 10 consecutive days. However, prolonged tamoxifen treatment did not further increase the fraction of tdTomato/S100beta double-positive astrocytes in astroSQS-tdTo animals (data not shown). Did potentially non-recombined (tdTomato negative) astrocytes proliferate? Cortical astrocytes expand by local proliferation within the first postnatal week (Ge et al., 2012) and astrocyte turnover in the healthy adult brain is limited (Smart and Leblond, 1961; McCarthy and Leblond, 1988). However, in order to exclude this possibility astroSQS-tdTo animals $(n=3)$ on cholesterol chow were treated with EdU starting from the first day of tamoxifen administration for 16 days to label proliferating cells. Confocal microscopy revealed that the vast majority of EdU positive cells were glial cells expressing NG2, indicating that these were oligodendrocyte precursor cells (OPC) which are known to proliferate throughout adulthood (Rivers et al., 2008; Young et al., 2013). In agreement with previous reports (Karram et al., 2008), we found some cells that were positive for S100beta and NG2. However, proliferating S100beta/NG2 double-positive cells were not enriched in tdTomato negative territories (Figure $8 \mathrm{E}$ ). These data show that proliferation of potentially non-recombined (tdTomato negative) astrocytes could not account for the reduction in tdTomato positive cells in astroSQS-tdTo animals.

\subsubsection{Is squalene synthase successfully inactivated in astrocytes of conditional mutants?}

In order to address this question, first the expression of Fdft1 and Hmgcr, the ratelimiting enzyme of cholesterol biosynthesis, as well as Apoe, the major apolipoprotein of the brain, was assessed by quantitative RT-PCR in the cortex of astroSQS-tdTo animals. Analysis revealed that the expression of Fdft1 and Hmgcr was unchanged in conditional mutants whereas the expression of Apoe was slightly but significantly increased (Figure $10 \mathrm{~B}$ ). With respect to the reduction in tdTomato positive astrocytes (see above) we next investigated recombination efficiency of the ROSA26 and Fdft1 loci on genomic DNA. 


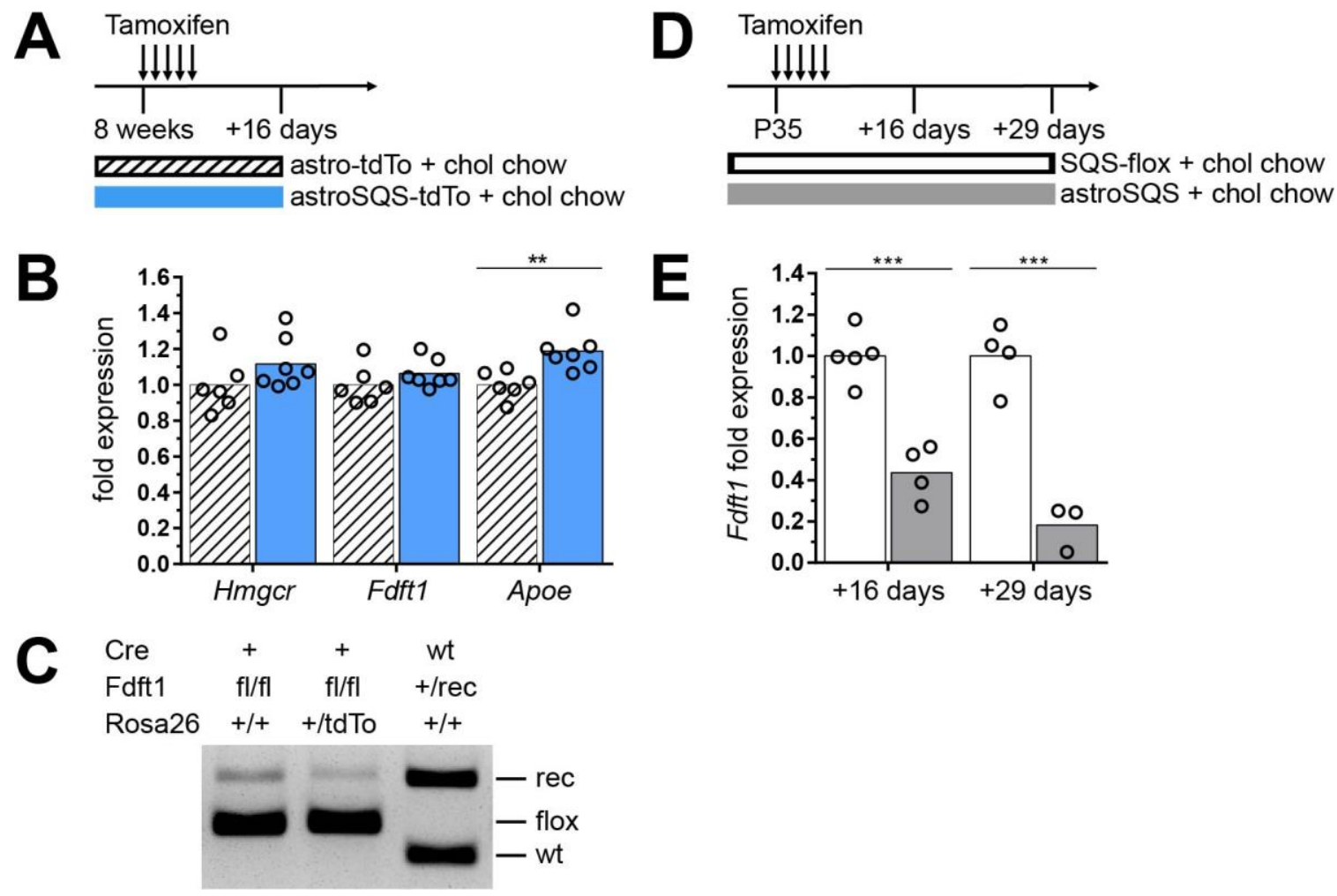

Figure 10: Evaluation of knock-out efficiency in conditional mutants. A) Scheme depicting the time course of tamoxifen administration and duration of cholesterol chow treatment. Male and female astro-tdTo and astroSQS-tdTo animals on cholesterol chow were analyzed 16 days after the first tamoxifen injection. B) Quantitative RT-PCR analysis of dissected cortices of astro-tdTo and astroSQS-tdTo mice determining the expression of genes involved in cholesterol production (Hmgcr, Fdft1) and cholesterol transport (Apoe). Bars represent mean fold expression normalized to astro-tdTo animals with individual data points $(n=6-7)$. C) PCR for Fdft1 alleles (rec: recombined, flox: floxed, wt: wild type) on genomic DNA isolated from cortices of astroSQS (left), astroSQS-tdTo (middle) animals at day 16 after the first tamoxifen injection. The right lane shows the PCR of a mouse harboring one floxed and one recombined Fdft1 allele. D) Scheme depicting the time course of tamoxifen administration and duration of cholesterol chow treatment. Male ( +16 days) and female (+29 days) SQS-flox and astroSQS animals on cholesterol chow were analyzed 16 days or 29 days after the first tamoxifen injection (P35). E) Quantitative RT-PCR analysis of MACS-isolated cortical astrocytes of SQS-flox and astroSQS animals at day 16 and day 29 after the first tamoxifen injection. Bars represent mean fold Fdft1 expression normalized to SQS-flox animals with individual data points $(n=3-5$ animals). Asterisks represent significant differences with ${ }^{* *} p<0.01,{ }^{* * *} p<0.001$ (unpaired Student's t-test). MACS isolation was performed together with Tim Düking. 
Semi-quantitative PCR in the cortex suggested that the abundance of recombined Fdft1 alleles was reduced in astroSQS-tdTo animals compared to astroSQS animals without the tdTomato reporter (Figure $10 \mathrm{C}$ ) although variation was high (56 $\pm 6 \%$ of recombined alleles in astroSQS-tdTo animals compared to $100 \pm 25 \%$ in astroSQS animals, $\mathrm{n}=4$ each, \pm s.e.m.). Therefore, Fdft1 recombination was assessed more directly on cellular level by isolating astrocytes from the cortex of astroSQS animals without the tdTomato reporter 16 and 29 days after tamoxifen injection by magnetic-activated cell sorting (MACS). Quantitative RT-PCR of isolated astrocytes revealed that Fdft1 expression was strongly down-regulated in astroSQS animals compared to SQS-flox mice 16 days and even further 29 days after tamoxifen injection (Figure $10 \mathrm{E}$ ) indicating successful recombination in the majority of astrocytes.

\subsubsection{What are the electrophysiological properties of mutant astrocytes?}

Electrophysiological assessment of astrocytes allows determining cell viability and maturation status (Kafitz et al., 2008; Dallérac et al., 2013). Proliferation in response to injury altered electrophysiological properties of astrocytes in vitro reminiscent of immature glial cells (MacFarlane and Sontheimer, 1997). We therefore determined electrophysiologically whether lack of cholesterol synthesis affects astrocyte viability and maturation. In adult astro-tdTo animals the electrophysiological properties of tdTomato expressing cells are characteristic of viable mature astrocytes (Winchenbach et al., 2016). In astroSQS-tdTo animals the presence of floxed Fdft1 and ROSA26-tdTomato alleles affected recombination leading to one population of astrocytes positive for tdTomato and one population without tdTomato expression (see above). 

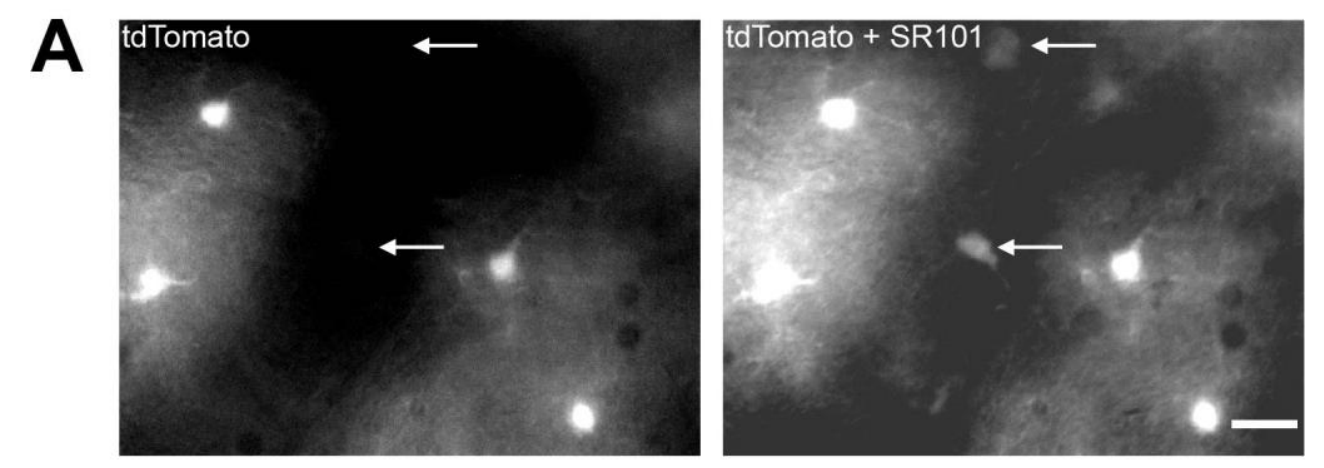

wild type: SR $101^{+}$astrocytes
astroSQS-tdTo: tdTomato ${ }^{+} \mathrm{SR} 101^{+}$astrocytes
astroSQS-tdTo: tdTomato- SR $101^{+}$astrocytes
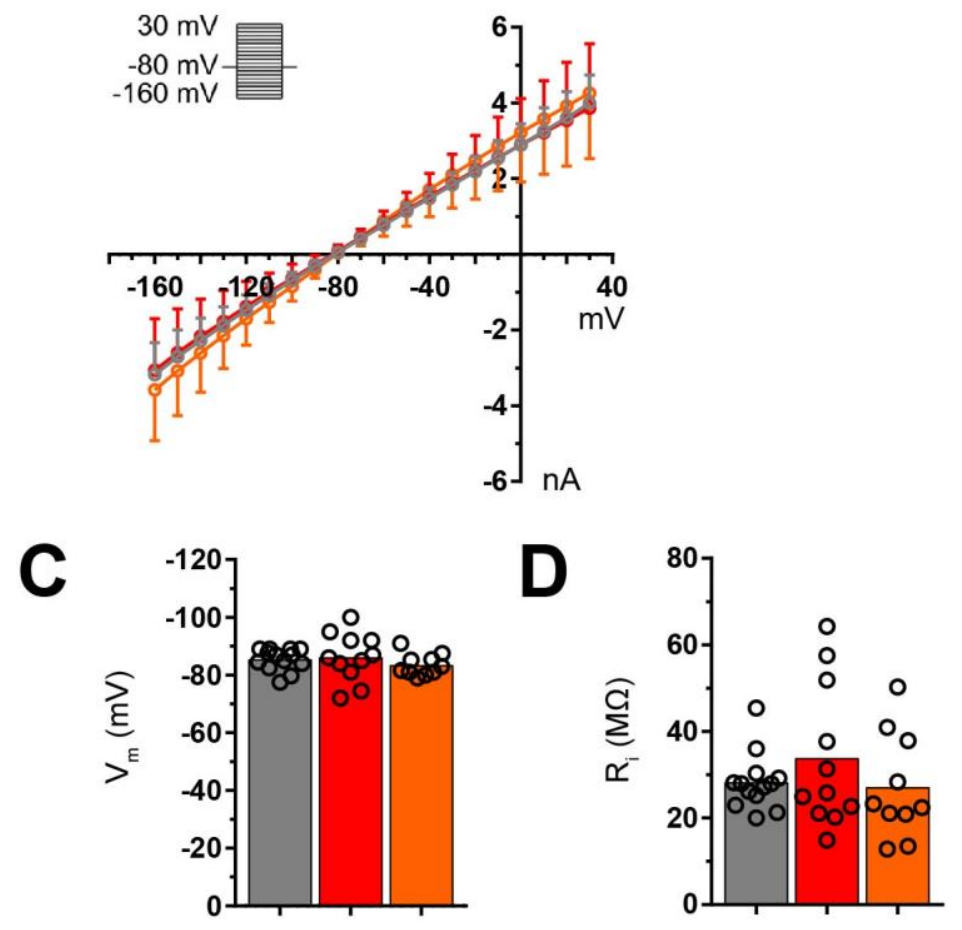

Figure 11: Electrophysiological properties of astrocytes in conditional mutants. A) Fluorescence images of acute forebrain slices stained with sulforhodamine 101 (SR101) to label astrocytes. Cortical astrocytes that did not express the Cre reporter tdTomato (arrows, left panel) were identified by SR101 staining (arrows, right panel). Scale, $20 \mu \mathrm{m}$. B) Wholecell patch clamp recordings were performed of cortical SR101 positive astrocytes of wild type animals (gray, $n=13$ cells), tdTomato/SR101 double-positive astrocytes ( $\mathrm{red}, \mathrm{n}=11$ cells) as well as tdTomato negative/SR101 positive astrocytes (orange, $\mathrm{n}=10$ cells) of astroSQS-tdTo animals. The graph shows averaged I-V curves for each cell population (mean \pm s.d.) in response to 200 ms voltage steps according to the voltage step protocol (inset). C) Resting membrane potential of astrocytes analyzed in B. Bars represent mean values with individual data points. D) Membrane resistance of astrocytes analyzed in $B$. Bars represent mean values with individual data points.

In order to target all astrocytes in the cortex, acute forebrain slices of adult astroSQS-tdTo animals were stained with sulforhodamine 101 (SR101) (Figure 11 
A). SR101 is a well-established marker to label astrocytes in various brain regions including cortex without altering the electrophysiological properties of the cells (Nimmerjahn et al., 2004; Kafitz et al., 2008; Schnell et al., 2012). Whole-cell patch clamp recordings revealed that both tdTomato positive and tdTomato negative astrocytes in astroSQS-tdTo animals did not differ from astrocytes of wild type animals in their response to varying voltage steps $(p=0.6234$ and $p=0.7676$, of max. inward and outward currents, respectively as assessed by one-way ANOVA) and displayed almost linear current/voltage relationships representative of mature astrocytes (Figure $11 \mathrm{~B}$ ). There was also no difference in resting membrane potential (Figure $11 \mathrm{C}$ ) and membrane resistance (Figure $11 \mathrm{D}$ ) between the cell populations indicating that all cells resembled viable mature astrocytes. These data indicate that lack of squalene synthase does not affect the electrophysiological properties of mature astrocytes.

\subsubsection{Does lack of squalene synthase in astrocytes affect blood-brain barrier integrity?}

Astrocytes cover nearly $100 \%$ of brain microvessels (Mathiisen et al., 2010) and are important for maintenance of blood-brain barrier (BBB) function including regulation of BBB integrity via ApoE (Bell et al., 2012). In order to assess whether lack of SQS impairs astrocyte function to maintain BBB integrity, brain water content as a measure of edema formation and the extent of Evans Blue (EB) extravasation was analyzed in the brains of astroSQS animals. Evans Blue is a fluorescent dye that binds serum albumin and a widely used marker to assess BBB integrity. As a positive control of assay sensitivity data of cuprizone fed animals are shown that have been published elsewhere (Berghoff et al., 2017a; 2017b). 

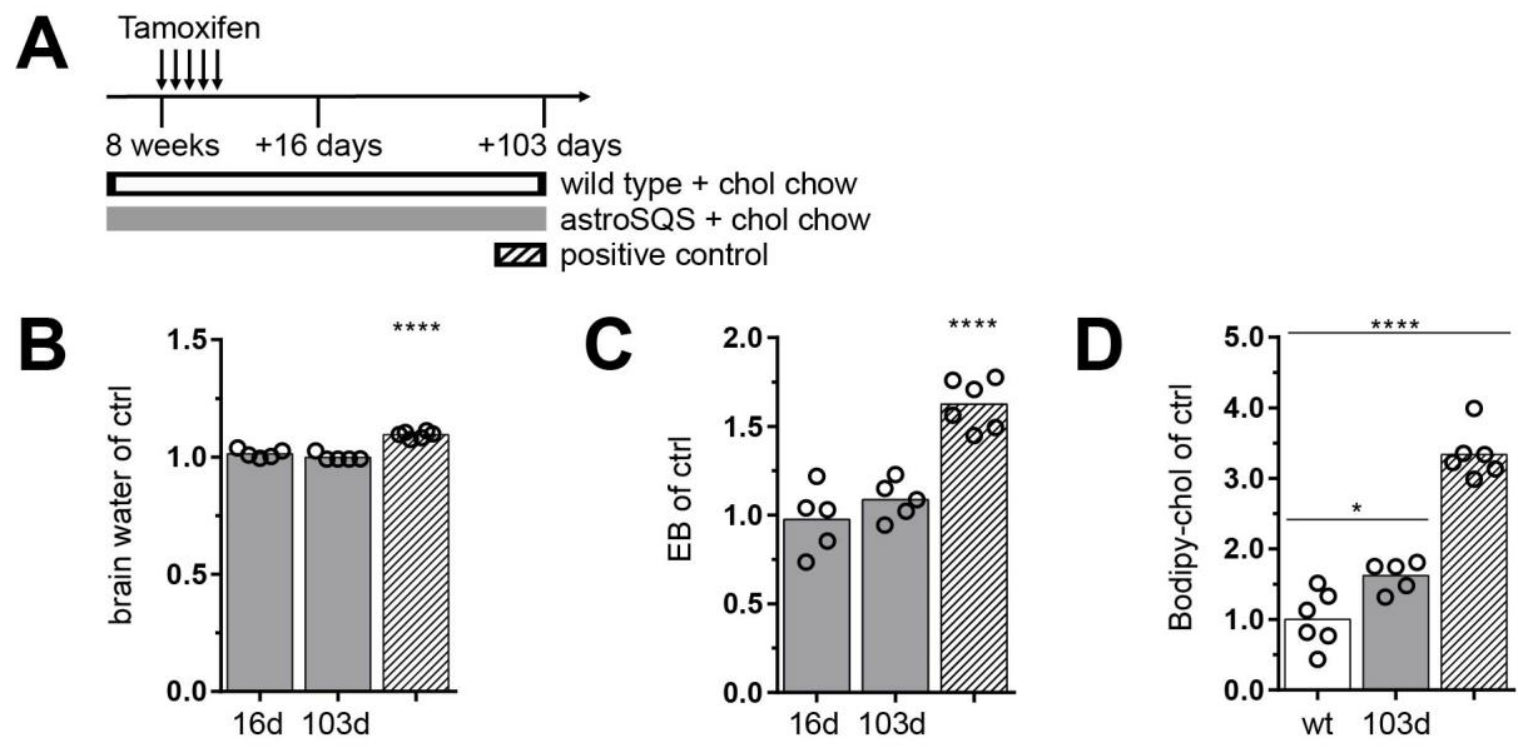
E Tamoxifen

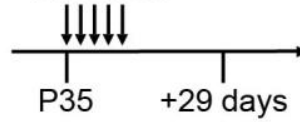
P35 +29 days
wild type + chol chow astroSQS + chol chow

$\mathbf{F}$

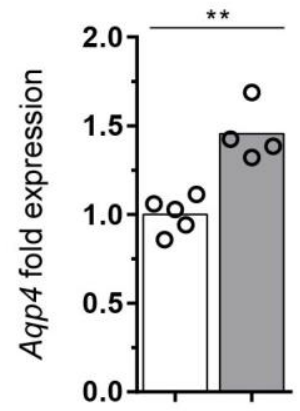

H

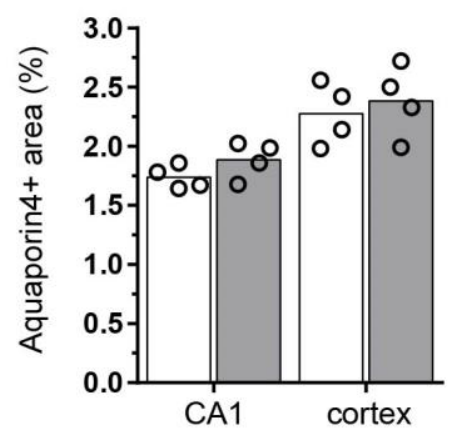

G
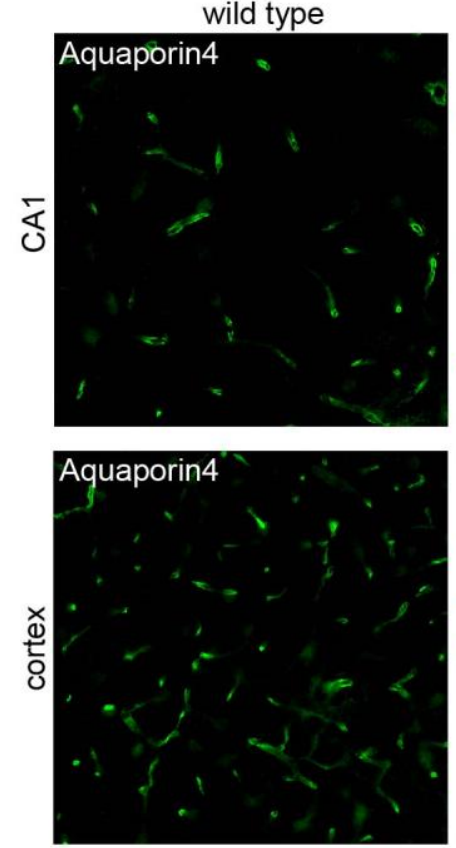

astroSQS
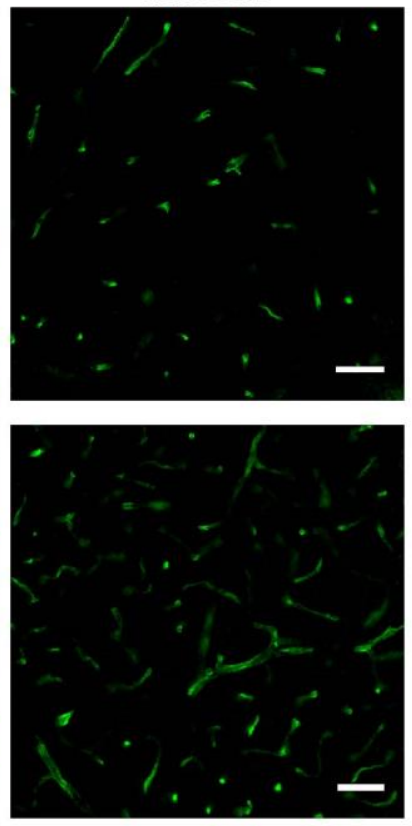
Figure 12: Blood-brain barrier integrity in conditional mutants. A) Scheme depicting the time course of tamoxifen administration and duration of cholesterol chow treatment. Wild type and astroSQS animals on cholesterol chow were analyzed 16 and 103 days after the first tamoxifen injection. As a positive control, data of cuprizone-fed animals that exhibit blood-brain barrier impairment are shown. Cuprizone data adapted from Berghoff et al., 2017a and Berghoff et al., 2017b. B) Brain water content of astroSQS animals 16 and 103 days after the first tamoxifen injection as well as brain water content of wild type animals fed a cuprizone diet for 5 weeks (Berghoff et al., 2017a). Bars represent mean values with individual data points normalized to corresponding control animals (16d: astroSQS, $n=5$, wild type control, $n=3$ animals; 103d: astroSQS, $n=5$, wild type control, $n=5$ animals; cuprizone: $n=6$, control, $n=5$ animals). C) Evans blue (EB) extravasation in brains of astroSQS animals 16 and 103 days after the first tamoxifen injection as well as EB extravasation of wild type animals fed a cuprizone diet for 5 weeks (Berghoff et al., 2017a). Bars represent mean values with individual data points normalized to corresponding control animals (16d: astroSQS, $n=5$, wild type control, $n=5$ animals; $103 d$ : astroSQS, $n=5$, wild type control, $n=5$ animals; cuprizone: $n=6$, control, $n=5$ animals). D) Extravasation of bodipy-cholesterol in brains of astroSQS animals 103 days after the first tamoxifen injection as well as of wild type animals fed a cuprizone diet for 5 weeks (Berghoff et al., 2017b). Bars represent mean values with individual data points normalized control animals (103d: astroSQS, $n=5$, cuprizone: $n=6$, wild type control, $n=6$ animals). E) Scheme depicting the time course of tamoxifen administration and duration of cholesterol chow treatment. Wild type and astroSQS animals on cholesterol chow were analyzed 29 days after the first tamoxifen injection. F) Quantitative RT-PCR analysis of dissected hippocampi of wild type ( $n=5$ animals) and astroSQS ( $n=4$ animals) 29 days after the first tamoxifen injection. Bars represent mean fold Aqp4 expression normalized to wild type animals with individual data points. G) Immunolabeling of Aquaporin4 in CA1 (upper row) and cortex (lower row) of wild type and astroSQS animals. Scales, $50 \mu \mathrm{m}$. H) Quantification of Aquaporin4 immunoreactivity in CA1 and cortex of wild type $(n=4)$ and astroSQS $(n=4)$ animals. Bars represent mean Aquaporin4 positive area (\%) with individual data points. Asterisks represent significant differences compared to corresponding controls with ${ }^{*} p<0.05,{ }^{* *} p<$ $0.01,{ }^{* * *} p<0.0001$ (unpaired Student's t-test). Analysis of cuprizone-fed animals was performed by Stefan Berghoff (Berghoff et al., 2017a; 2017b). Analysis of EB and bodipycholesterol extravasation ( $C$ and $D$ ) was performed together with Stefan Berghoff: Jan Winchenbach performed tamoxifen injections, tissue preparation and lyophilization. Stefan Berghoff performed tracer injection, formamide extraction and tracer quantification.

In astroSQS animals brain water content and the amount of extravasated EB were unaffected 16 and 103 days after tamoxifen injection compared to wild type animals and in contrast to cuprizone fed animals that display edema formation and BBB dysfunction (Figure 12 B, C, D and Berghoff et al., 2017a; 2017b). To assess whether peripheral cholesterol enters the brain, extravasation of the fluorescent cholesterol derivative bodipy-cholesterol was determined in the brains of astroSQS animals. After intraperitoneal injection slightly but significantly increased extravasation of bodipy-cholesterol was detected in astroSQS animals (Figure 12 D). Dysregulation of the water channel aquaporin 4 (AQP4) expressed on astroglial endfeet occurs during edema formation and increased Aqp4 abundance indicates BBB impairment (Abbott et al., 2005). In younger astroSQS animals Aqp4 
expression was increased in the hippocampus compared to wild type mice (Figure $12 \mathrm{E}, \mathrm{F})$ but protein levels were unaltered in the CA1 region of the hippocampus and cortex as assessed by immunolabeling (Figure $12 \mathrm{G}, \mathrm{H}$ ). Together, these data indicate that astroSQS animals do not display BBB breakdown but peripheral cholesterol might cross the BBB at low levels.

\subsection{Does lack of astrocytic cholesterol synthesis affect Alzheimer's disease pathology?}

The successful targeting of the majority of CNS astrocytes and the lack of pathological changes render astroSQS mutants a useful tool to study the effect of astrocyte-specific loss of cholesterol synthesis on Alzheimer's disease (AD) pathology. The 5xFAD mouse model of $A D$ displays progressive deposition of amyloid- $\beta$ peptides $(A \beta)$ starting in cortex and subiculum of the hippocampus at around 2 months of age accompanied by astrocyte and microglia activation (Oakley et al., 2006). Memory deficits become apparent at 6 months and progress with age accompanied by neuron loss in cortical layer $V$ at 12 months (Jawhar et al., 2012).

\subsubsection{Are cholesterol homeostasis and BBB integrity altered in 5xFAD animals?}

In order to assess whether cholesterol homeostasis is altered in 5xFAD animals, the expression of the major genes involved in cholesterol synthesis (Figure $13 \mathrm{~A}$ ), transport (Figure $13 \mathrm{~B}$ ), uptake (Figure $13 \mathrm{C}$ ) and excretion (Figure $13 \mathrm{D}$ ) was analyzed in the hippocampus of 5xFAD animals at postnatal day 64 (P64). Expression levels were unchanged compared to age and sex-matched wild type animals except for a slight reduction in the expression of apolipoprotein $\mathrm{J}$ (also known as clusterin, $\mathrm{Clu}$ ). To analyze whether peripheral cholesterol could potentially affect brain cholesterol homeostasis, we next assessed BBB integrity in 5xFAD animals. There was no change in brain water content and only minor increase in extravasated EB and sodium fluorescein (NaFI) during advanced disease in 4.5 month old female 5xFAD mice (Figure $13 \mathrm{E}-\mathrm{G}$ ). 

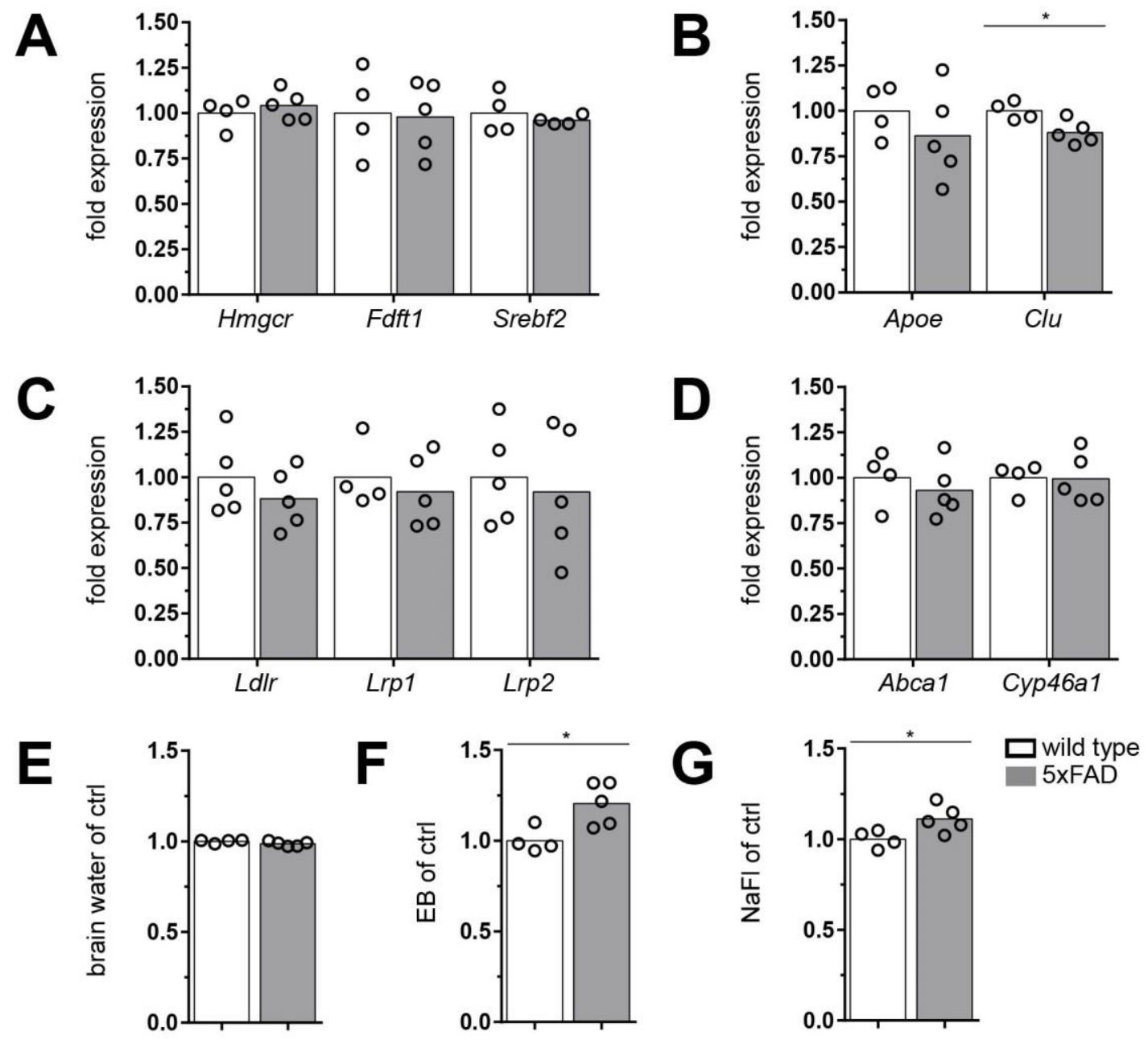

Figure 13: Cholesterol homeostasis and BBB integrity in 5xFAD animals. Male wild type and 5xFAD animals received tamoxifen injections on 5 consecutive days starting at P35 and were treated with $0.5 \%$ cholesterol chow until analysis at day 29 after the first tamoxifen injection (A-D). Quantitative RT-PCR analysis of dissected hippocampi of wild type and 5xFAD animals was performed to assess expression of genes involved in cholesterol production (A), transport (B), uptake (C) and excretion (D). Bars represent mean fold expression normalized to wild type animals with individual data points $(n=4-5)$. Assessment of BBB integrity was performed in female 4.5 month old 5xFAD and age-matched female wild type animals on normal chow without tamoxifen administration. E) Brain water content of wild type and 5xFAD animals. Bars represent mean values with individual data points normalized to wild type animals (wild type, $n=4,5 x F A D, n=5$ ). $F$ ) Evans blue (EB) extravasation in brains of wild type and 5xFAD animals. Bars represent mean values with individual data points normalized to wild type animals (wild type, $n=4,5 x F A D, n=5$ ). G) Extravasation of sodium fluorescein ( $\mathrm{NaFI}$ ) in brains of wild type and 5xFAD animals. Bars represent mean values with individual data points normalized control animals (wild type, $n=4,5 x F A D, n=5$ ). Asterisks represent significant differences with ${ }^{*} p<0.05$ (unpaired Student's t-test). Analysis of $\mathrm{EB}$ and $\mathrm{NaFl}$ extravasation ( $\mathrm{F}$ and $\mathrm{G}$ ) was performed together with Stefan Berghoff: Jan Winchenbach performed tissue preparation and lyophilization. Stefan Berghoff performed tracer injection, formamide extraction and tracer quantification. 


\subsubsection{Conditional inactivation of squalene synthase in 5xFAD animals}

In order to inactivate squalene synthase in 5xFAD animals, astroSQS mice were crossed with 5xFAD mice generating offspring with the genotype Aldh111CreERT2*5xFAD*Fdft $1^{\text {flox/flox }}$, hereafter termed astroSQS-5xFAD mutants. Age and sex-matched wild type, astroSQS and 5xFAD animals served as controls. All animals received $0.5 \%$ cholesterol chow and were injected with tamoxifen at P35 and analyzed 29 days later (Figure $14 \mathrm{~A}$ ) because preliminary experiments indicated early changes in astroSQS-5xFAD mutants (data not shown). Due to the different extent of amyloid pathology (Oakley et al., 2006) male and female mice were analyzed separately. Conditional astroSQS-5xFAD mutants were healthy with normal body weight (Figure $14 \mathrm{~B}, \mathrm{C}$ ) and did not show any obvious behavioral abnormalities. There was also no change in serum cholesterol concentration in conditional mutants (Figure $14 \mathrm{D}, \mathrm{E}$ ).
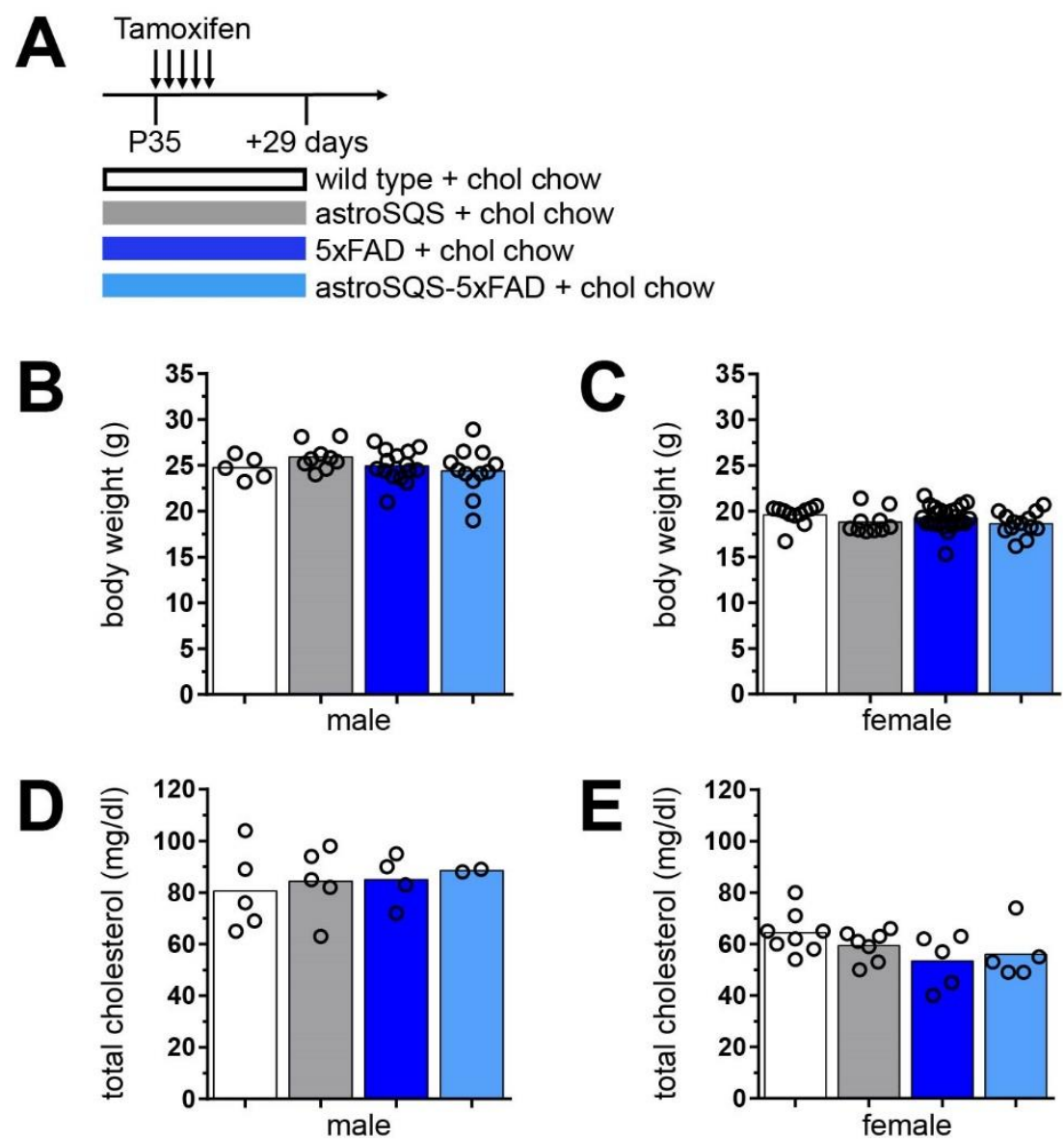
Figure 14: Body weight and serum cholesterol unchanged in astroSQS-5xFAD mutants. A) Scheme depicting the time course of tamoxifen administration and duration of cholesterol chow treatment. Wild type, astroSQS as well as 5xFAD and compound astroSQS-5xFAD mutants were analyzed 29 days after the first tamoxifen injection. Male and female mice were analyzed separately. Body weight (g) of male (B) and female (C) mice was not different in all experimental groups analyzed. Bars represent mean values with individual data points (wild type: male, $n=5$, female, $n=11$; astroSQS: male, $n=9$, female, $n=10 ; 5 x F A D$ : male, $n=15$, female, $n=24$; astroSQS-5xFAD: male, $n=12$, female, $n=13)$. Total serum cholesterol concentration $(\mathrm{mg} / \mathrm{dl})$ of male $(D)$ and female $(E)$ mice was not different in all experimental groups analyzed. Bars represent mean values with individual data points (wild type: male, $n=5$, female, $n=8$; astroSQS: male, $n=5$, female, $n=7$; 5xFAD: male, $n=4$, female, $n=5$; astroSQS-5xFAD: male, $n=2$, female, $n=5$ ).

\subsubsection{Does lack of astrocytic cholesterol synthesis affect cerebral $\beta$ - amyloidosis?}

In 5xFAD mice $A \beta$ deposition starts in the subiculum and cortical layers IV to $\mathrm{VI}$ at around 2 months of age (Oakley et al., 2006). To assess A $\beta$ deposition in astroSQS5XFAD mice serial sections throughout the hippocampus were immunolabeled using a pan $A \beta$ antibody (218211, clone NT244). Microscopy revealed extracellular A $\beta$ deposits as well as some intracellular immunoreactivity restricted to the subiculum of the hippocampus (data not shown). Quantification showed reduced $A \beta$ immunoreactivity throughout the hippocampus in astroSQS-5xFAD mice $(0.44 \pm$ $0.09 \% A \beta$ positive area) compared to 5xFAD controls $(0.56 \pm 0.05 \%, n=5-6$ animals each, \pm s.e.m.) without reaching statistical significance. In order to quantify $A \beta$ deposition more directly, hippocampal sections were immunolabeled with an A 342 specific antibody (44-344, ThermoFisher) that detected extracellular deposits without intracellular staining (Figure $15 \mathrm{~A}, \mathrm{~B}$ ). Quantification revealed significantly reduced $A \beta 42$ deposition in the subiculum of male astroSQS-5xFAD mice compared to $5 x F A D$ controls (Figure $15 \mathrm{C}$ ). Non-plaque oligomeric $A \beta$ species are increased in $A D$ patients (McDonald et al., 2012) and accumulating evidence indicate $A \beta$ oligomers in neurotoxicity and cognitive decline (reviewed in Aguzzi and O'Connor, 2010). Therefore, water- (TBS-soluble) and detergent-soluble (SDS-soluble) A $\beta 40$ and $A \beta 42$ peptides were quantified in the hippocampus of astroSQS-5xFAD animals. There was no difference in the concentration of TBS-soluble and SDSsoluble $A \beta$ peptides in male (Figure $15 \mathrm{D}, \mathrm{F}$ ) and female (Figure $15 \mathrm{E}, \mathrm{G}$ ) astroSQS- 
5xFAD mice compared to 5xFAD controls. These findings show that albeit soluble $A \beta$ levels are unchanged, $A \beta 42$ deposition is reduced in astroSQS-5XFAD animals.

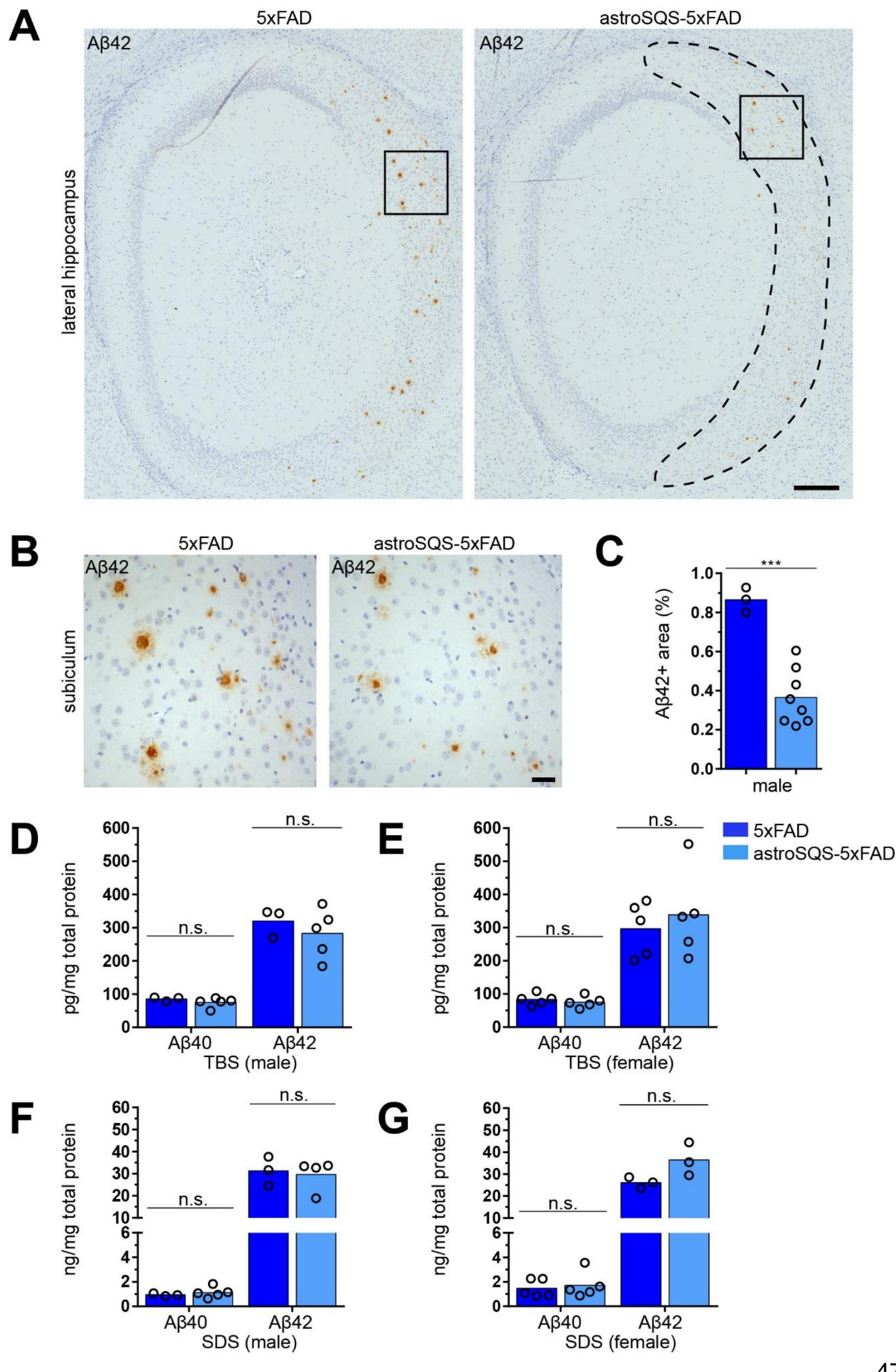


Figure 15: Compound astroSQS-5xFAD mutants exhibit reduced $A \beta 42$ deposition in the subiculum. A) Representative pictures of $A \beta 42$ immunolabeling of the lateral hippocampus (sagittal sections) of male 5XFAD (left panel) and astroSQS-5xFAD (right panel) animals at P64 (29 days after the first tamoxifen injection) with the subiculum outlined as dashed line (right panel). Scale, $300 \mu \mathrm{m}$. B) A 342 immunolabeling in the subiculum of 5xFAD and astroSQS-5xFAD animals at locations indicated in (A). Scale, $40 \mu \mathrm{m}$. C) Quantification of $A \beta 42$ deposition in the subiculum of male 5xFAD and astroSQS-5xFAD animals at $P 64$. Bars represent mean $A \beta 42$ positive area (\%) with individual data points (5xFAD, $n=3$, astroSQS-5xFAD, $n=8$ ). TBS-soluble ( $D$ and $E$ ) and SDS-soluble ( $F$ and G) $A \beta 40$ and $A \beta 42$ peptides were quantified in the hippocampus of male (D and $F$ ) and female ( $E$ and $G$ ) 5xFAD and astroSQS-5xFAD mice at P64 using an electrochemiluminescene-linked immunoassay. Bars represent mean values normalized to total protein concentration with individual data points (5xFAD: male, $n=3$, female, $n=3-5$; astroSQS-5xFAD: male, $\mathrm{n}=4-5$, female, $\mathrm{n}=3-5)$. Asterisks represent significant differences with ${ }^{* * *} p<0.001$; n.s., not significant (unpaired Student's t-test).

\subsubsection{Is the reduction in $A \beta$ deposition due to increased clearance by glial cells?}

Neuroinflammation with activation of astrocytes and microglia is a common feature in the brains of $A D$ patients and mouse models of $A D$ and both cell types are able to degrade $A \beta$ (reviewed in Solito and Sastre, 2012; Ferrer, 2017). ApoE promotes the degradation of $A \beta$ by astrocytes and microglia in vitro (Koistinaho et al., 2004; Jiang et al., 2008). Promoting lipidation of $A p o E$ increased $A \beta$ clearance by microglia in vitro and reduced $A \beta$ deposition in vivo (Jiang et al., 2008). Histological analysis of lba1 immunoreactivity is a widely used method to assess microglia activation in 5xFAD mice (Oakley et al., 2006; Katsouri and Georgopoulos, 2011; Wang et al., 2015; Marsh et al., 2016). Similar to lba1 expression, increase in GFAP immunoreactivity has been demonstrated in vicinity of $A \beta$ deposits and with disease progression in 5xFAD mice indicating astrogliosis (Oakley et al., 2006; Katsouri and Georgopoulos, 2011; Hüttenrauch et al., 2015). In order to assess astroglial and microglial responses in astroSQS-5XFAD mice, hippocampal sections were immunolabeled for GFAP and lba1. As expected, hypertrophic microglia with enlarged cell bodies were found in the immediate vicinity of $A \beta$ deposits (Figure 16 $A$, arrows) in the subiculum whereas hypertrophic astrocytes were either found closely associated with or located in proximity to plaques (Figure $16 \mathrm{~A}$ ). 

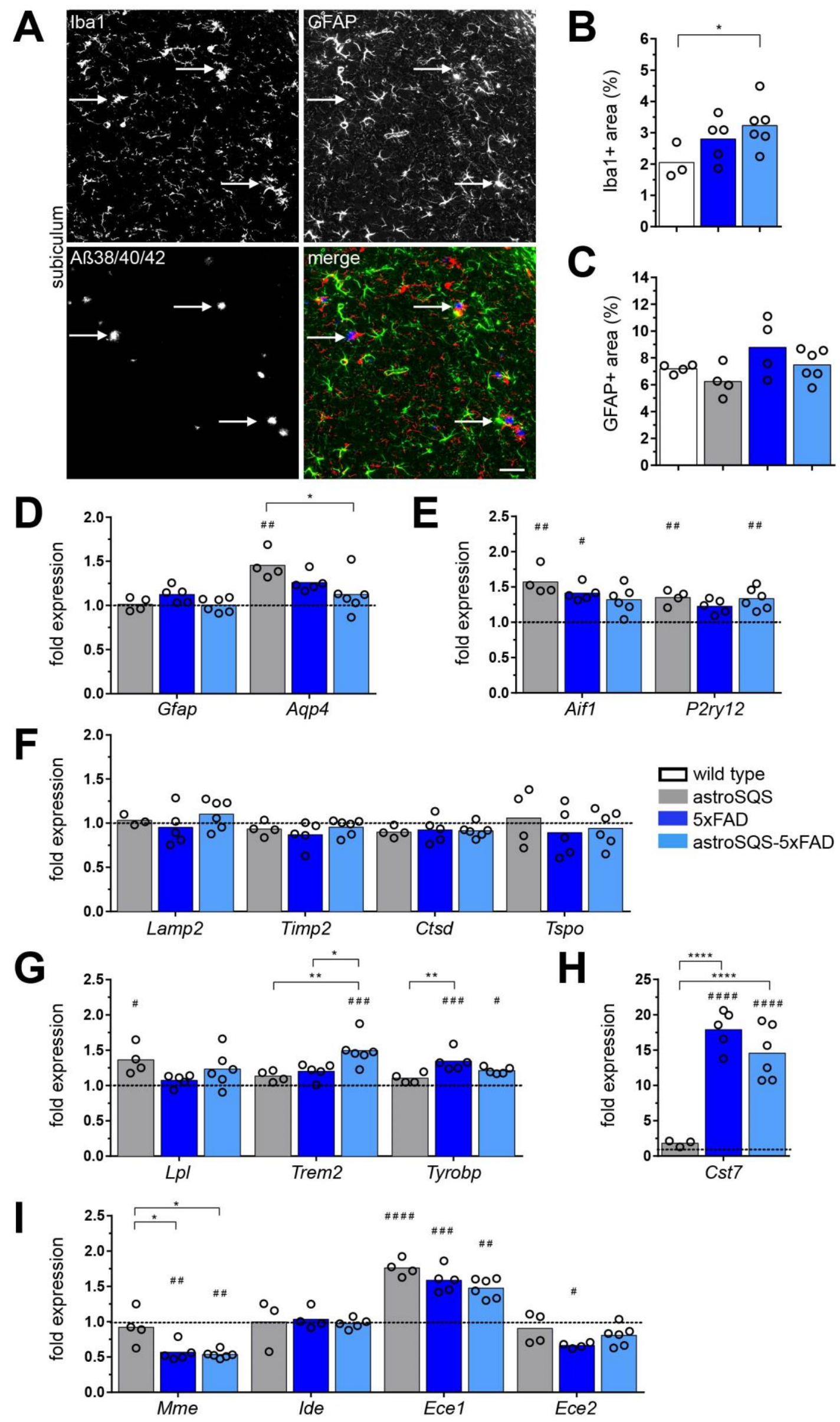
Figure 16: Astroglial and microglial response in compound astroSQS-5xFAD mutants. A) Co-immunolabeling of microglia (lba1, red), astrocytes (GFAP, green) and A $\beta$ ( $A \beta 38 / 40 / 42$, labeled with pan anti-A $\beta$ antibody, blue) with location of $A \beta$ deposits indicated (arrows) in the subiculum of male astroSQS-5xFAD mice at P64. Scale, 50 $\mu$ m. B) Quantification of Iba1 positive microglia in the subiculum of male wild type $(n=3), 5 x F A D$ $(n=5)$ and astroSQS-5xFAD $(n=6)$ animals at P64. Bars represent mean lba1 positive area (\%) with individual data points. C) Quantification of GFAP positive astrocytes in the subiculum of male wild type $(n=4)$, astroSQS $(n=4), 5 x F A D(n=4)$ and astroSQS-5xFAD $(n=6)$ animals at P64. Bars represent mean GFAP positive area $(\%)$ with individual data points. Quantitative RT-PCR analysis of dissected hippocampi of male wild type $(n=4-5)$, astroSQS $(n=3-4)$, 5xFAD $(n=4-5)$ and astroSQS-5xFAD $(n=5-6)$ animals at P64 determining the expression of genes involved in astrocyte activation and function (D), microglia activation and homeostasis (E), as well as genes involved in microgliosis, phagocytosis, risk for Alzheimer's disease (F-H) and degradation of $A \beta(\mathbf{I})$. Bars represent mean fold expression normalized to wild type animals (set to 1, dashed line) with individual data points. Asterisks represent significant differences with ${ }^{*} p<0.05,{ }^{* *} p<0.01,{ }^{* * * *} p<$ 0.0001 , hash symbols denote significant difference relative to wild type controls with $\# p<$ $0.05, \# \# p<0.01, \# \# \# p<0.001$, \#\#\#\#p <0.0001 (one-way ANOVA with Tukey's multiple comparison test). Immunohistochemical analysis (A-C) was performed together with Borja Gómez Ramos: Jan Winchenbach performed tamoxifen injections, tissue preparation and fixation as well as quantification. Borja Gómez Ramos performed most of the immunolabeling and microscopy.

Quantification revealed elevated lba1 immunoreactivity in the subiculum of 5xFAD mice which was significantly increased in astroSQS-5XFAD animals compared to wild type (Figure $16 \mathrm{~B}$ ). Although GFAP immunoreactivity was increased locally at plaques in 5XFAD and astroSQS-5XFAD animals, quantification did not reveal significant changes throughout the subiculum compared to wild type and astroSQS animals (Figure $16 \mathrm{C}$ ). Quantitative RT-PCR analysis in the hippocampus showed also no differences in the expression of Gfap while Aqp4 expression was increased in astroSQS mice but not in astroSQS-5xFAD mice compared to wild type animals (Figure $16 \mathrm{D}$ ). Analysis of genes involved in microglia activation and homeostasis revealed increased expression of the gene encoding lba1 (allograft inflammatory factor 1, Aif1) in 5xFAD mice as previously reported (Landel et al., 2014) but also in astroSQS mice (Figure $16 \mathrm{E}$ ). Expression of P2ry12 (purinergic receptor P2Y12), a homeostatic microglia marker that is down-regulated upon cellular activation in response to CNS injury (Haynes et al., 2006) and in disease-associated microglia in 5xFAD mice (Keren-Shaul et al., 2017) was significantly increased in astroSQS and astroSQS-5xFAD animals compared to wild type animals (Figure $16 \mathrm{E}$ ). In order to assess microglial changes in more detail, the expression of genes involved in microglia activation and phagocytosis as well as genes involved in increased risk 
for $A D$ was quantified. Expression of several genes was found to be unchanged including Lamp2 (lysosome-associated membrane protein 2, also known as Mac3), Timp2 (tissue inhibitor of metalloproteinases 2), Ctsd (cathepsin D) and Tspo (translocator protein, Figure $16 \mathrm{~F}$ ). The expression of $L p /$ (lipoprotein lipase), an enzyme involved in lipid metabolism found in activated microglia near $A \beta$ plaques in 5xFAD mice and AD patients (Keren-Shaul et al., 2017), was increased in astroSQS mice but not in 5xFAD and astroSQS-5xFAD mice (Figure $16 \mathrm{G}$ ). Due to its role in the risk for $A D$ and $A \beta$ clearance (reviewed in Ulrich et al., 2017) the expression of Trem2 (triggering receptor expressed on myeloid cells 2), a lipid sensor expressed by microglia, and its signaling adaptor DAP12 (encoded by the Tyrobp gene) were analyzed. Expression of Trem2 was found to be significantly increased in astroSQS5xFAD mice compared to 5xFAD mice while the expression of Tyrobp was elevated both in astroSQS-5xFAD and 5xFAD animals (Figure $16 \mathrm{G}$ ). The strongest increase in expression level both in 5XFAD and astroSQS-5XFAD mice was detected for the lysosomal protease inhibitor cystatin $F$ (encoded by Cst7) in line with its increase in plaque-associated microglia in 5xFAD mice (Ofengeim et al., 2017). However, expression was not different in astroSQS-5XFAD compared to 5XFAD animals (Figure $16 \mathrm{H}$ ). Next, the expression of $A \beta$ degrading enzymes was analyzed in astroSQS-5XFAD animals. As previously reported for 5xFAD animals at similar age the expression of $M m e$ (encoding neprilysin) was reduced compared to wild type mice (Py et al., 2014; Hüttenrauch et al., 2015) but unchanged between 5xFAD and astroSQS-5XFAD animals (Figure $16 \mathrm{l}$ ). There was also no difference in the expression of Ide (insulin-degrading enzyme), Ece1 and Ece2 (endothelinconverting enzyme 1 and 2) between 5xFAD and astroSQS-5xFAD mice (Figure 16 I). Together these data show, that changes in astroglial and microglial responses between 5xFAD and astroSQS-5xFAD animals so far seem to be limited to Trem2 expression.

\subsubsection{Is the cholesterol metabolism altered in astroSQS-5XFAD mutants?}

To assess the effect of SQS inactivation in astrocytes on brain cholesterol metabolism in wild type and 5xFAD animals, hippocampal expression of genes involved in cholesterol production, transport, secretion and uptake was analyzed by 
quantitative RT-PCR. The expression of the major enzymes regulating cholesterol production were unchanged in all experimental groups (Figure $17 \mathrm{~A}$ ). The expression of Apoe was variable between individual animals and unchanged across genotypes, while Clu was slightly down-regulated in 5xFAD and astroSQS-5xFAD mice compared to astroSQS but not to wild type animals (Figure $17 \mathrm{~B}$ ). There were no changes in mRNA levels of genes involved in cellular cholesterol excretion (Figure $17 \mathrm{C}$ ) and uptake of cholesterol (Figure $17 \mathrm{D}$ ). Furthermore, western blot analysis revealed no changes in ApoE protein abundance in the hippocampus across genotypes (Figure $17 \mathrm{E}, \mathrm{F}$ ). These data suggest that lack of SQS in astrocytes does not lead to drastic changes in tissue cholesterol homeostasis in wild type or 5xFAD animals.

\subsubsection{Is APP processing to generate A $\beta$ altered in astroSQS-5XFAD mutants?}

In 5xFAD mice expression of mutant human APP and PS1 and therefore A $\beta$ generation is directed to neurons by the Thy 1 promoter (Oakley et al., 2006). Neuronal amyloidogenic processing of APP in endosomes is affected by cellular cholesterol content in vitro (Simons et al., 1998; Wahrle et al., 2002; Schneider et al., 2008) and direct interaction of APP and cholesterol could enhance $\beta$-secretase mediated $A \beta$ production in cholesterol-rich lipid rafts (Barrett et al., 2012). We therefore asked whether lack of astrocytic cholesterol support to neurons could modulate neuronal cholesterol content that, in turn could affect APP processing. As expected, expression of murine App was not affected by SQS inactivation or 5xFAD transgene expression (Figure $18 \mathrm{~A}$ ). In contrast to previous reports (Zhao et al., 2007) expression of Bace1 (beta-site APP-cleaving enzyme 1), the major $\beta$ secretase cleaving APP (Hussain et al., 1999; Sinha et al., 1999; Vassar et al., 1999; Yan et al., 1999) was slightly reduced in 5xFAD compared to wild type animals (Figure $18 \mathrm{~A}$ ). 

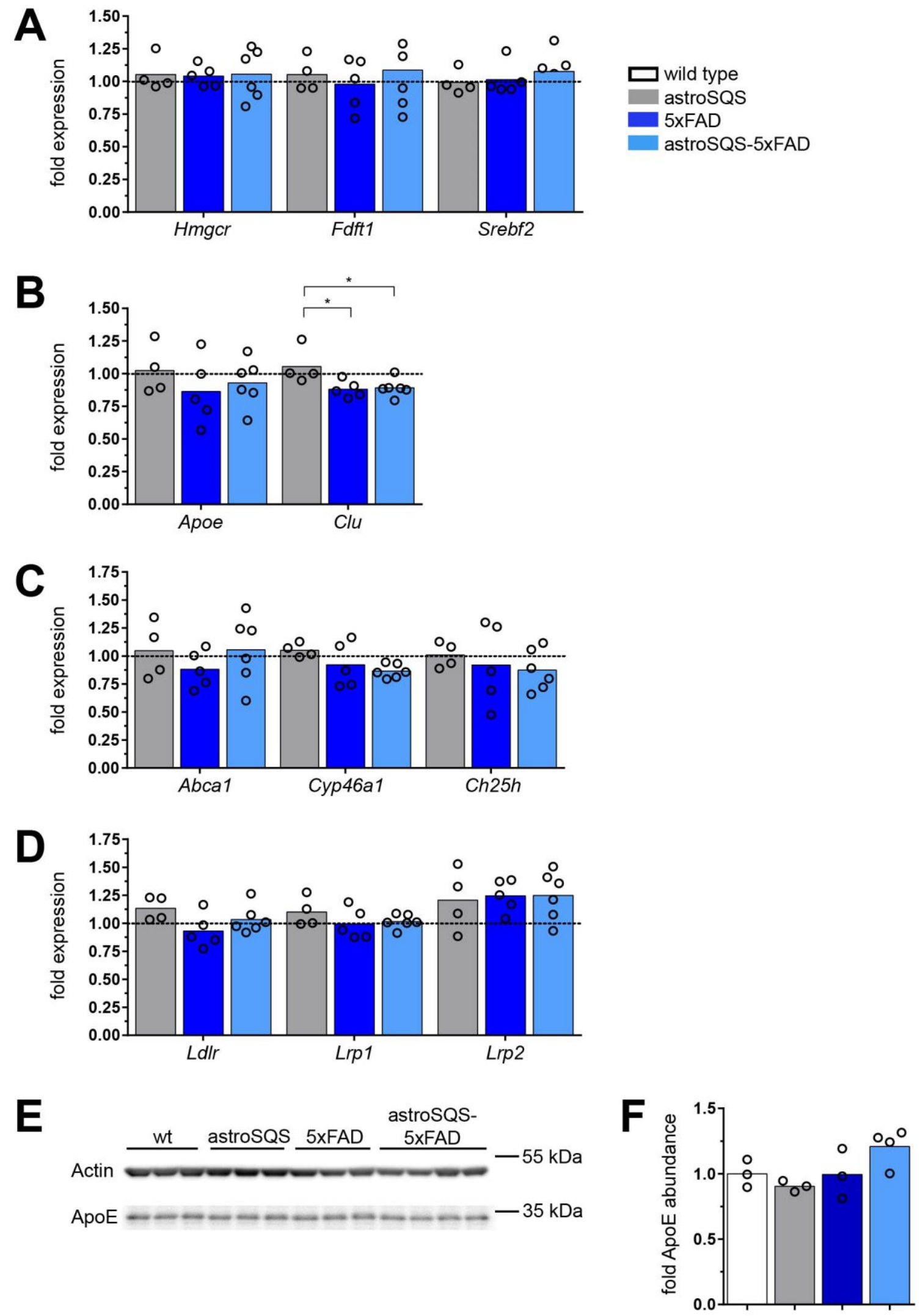
Figure 17: Cholesterol homeostasis is unchanged in compound astroSQS-5xFAD mutants. Quantitative RT-PCR analysis of dissected hippocampi of male wild type $(\mathrm{n}=4$ 5), astroSQS $(n=4), 5 x F A D(n=5)$ and astroSQS-5xFAD $(n=5-6)$ animals at P64 was performed to assess expression of genes involved in cholesterol production (A), transport (B), uptake (C) and excretion (D). Bars represent mean fold expression normalized to wild type animals (set to 1, dashed line) with individual data points. Asterisks represent significant differences with ${ }^{*} p<0.05$ (one-way ANOVA with Tukey's multiple comparison test). E) Western blot analysis for Apolipoprotein $E$ (ApoE) of dissected hippocampi of male wild type $(n=4)$, astroSQS $(n=3), 5 x F A D(n=3)$ and astroSQS-5xFAD $(n=4)$ animals at P64. Wt, wild type; kDa, kilodalton. F) Densitometric quantification of ApoE bands normalized to Actin loading control shown in $(E)$. Bars represent mean ApoE protein abundance normalized to wild type animals with individual data points

Bace2, a Bace1 homologue was found to also cleave APP at the $\beta$-site but more prominently at the $\alpha$-site (Hussain et al., 2000; Yan et al., 2001). Overexpression of Bace 2 reduced $A \beta$ production in vitro and Bace2 could act as an $A \beta$ degrading enzyme (Sun et al., 2006; Abdul-Hay et al., 2012). Surprisingly, Bace2 expression was significantly reduced in 5xFAD compared to astroSQS-5xFAD mice (Figure 18 A). Next, the abundance of APP C-terminal fragments resulting from $\beta$-secretase (C99 or $\beta-C T F)$ and $\alpha$-secretase cleavage (C83 or $\alpha-C T F)$ of APP were analyzed in the hippocampus of 5xFAD and astroSQS-5xFAD animals (Figure $18 \mathrm{~B}$ ). The abundance of C99 and C83 fragments was unchanged in male astroSQS-5XFAD mice whereas C99 abundance was significantly elevated in female astroSQS5xFAD animals compared to 5xFAD mice (Figure $18 \mathrm{C}, \mathrm{D}$ ). However, despite significant variation between animals the abundance of C83 was also increased in female astroSQS-5xFAD mice resulting in an unchanged C83/C99 ratio (Figure 18 $D, E)$. Together these findings suggest that lack of astrocytic cholesterol production does not affect neuronal APP processing. In summary, inactivation of cholesterol synthesis in astrocytes resulted in reduced $A \beta 42$ deposition in the subiculum without obvious changes in tissue cholesterol homeostasis or APP processing. Whether lack of neuronal cholesterol synthesis differently affects APP processing and A $\beta$ deposition is addressed in the next paragraph. 


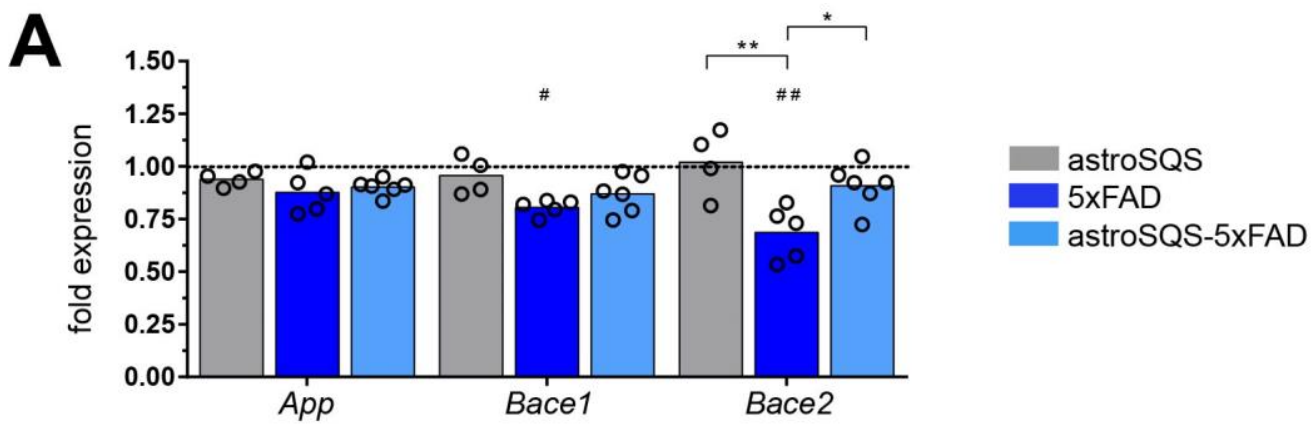

B
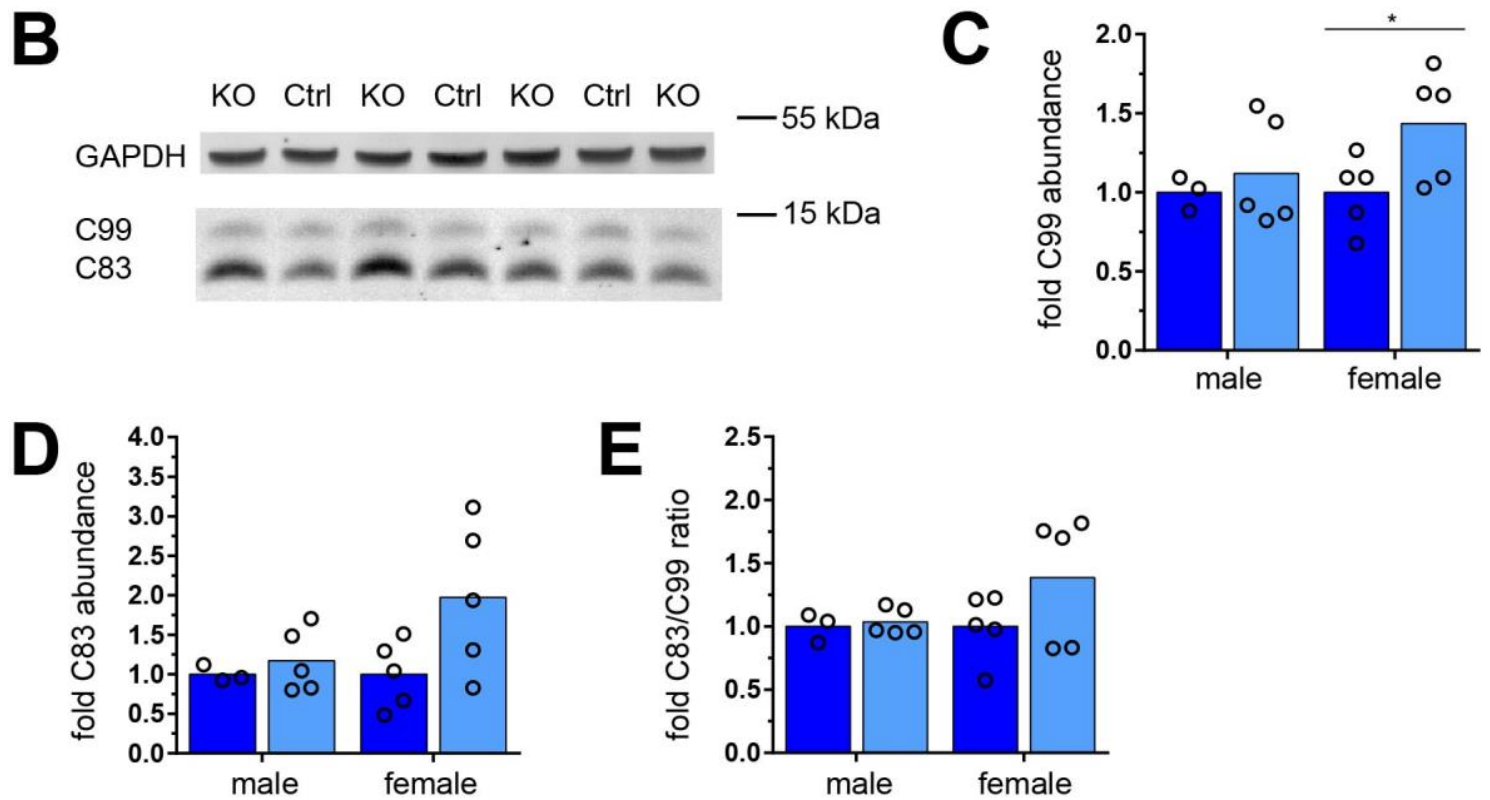

Figure 18: Assessment of APP processing in compound astroSQS-5xFAD mutants. A) Quantitative RT-PCR analysis of dissected hippocampi of male wild type $(n=4-5)$, astroSQS $(n=4), 5 x \operatorname{xAD}(n=5)$ and astroSQS-5xFAD $(n=6)$ animals at P64 was performed to assess expression of murine amyloid precursor protein (App) and APP protein processing enzymes (Bace1, Bace2). Bars represent mean fold expression normalized to wild type animals (set to 1, dashed line) with individual data points. Asterisks represent significant differences with ${ }^{*} p<0.05,{ }^{* *} p<0.01$, hash symbols denote significant difference relative to wild type controls with \#p $<0.05$, \#\#p $<0.01$ (one-way ANOVA with Tukey's multiple comparison test). B) Western blot analysis of APP C-terminal fragments C99 and C83 of dissected hippocampi (SDS-soluble protein fraction) of male 5xFAD and astroSQS5xFAD animals at P64. KO, knock-out (astroSQS-5xFAD); Ctrl, control (5xFAD); kDa, kilodalton. Densitometric quantification of C99 (C) and C83 (D) bands normalized to GAPDH loading control of male and female $5 \times$ FAD (male, $n=3$, female, $n=5$ ) and astroSQS-5xFAD (male, $\mathrm{n}=5$, female, $\mathrm{n}=5$ ) animals. Bars represent mean C99 (C) and C83 (D) protein abundance normalized to 5xFAD animals with individual data points. E) Ratio of C83/C99 protein abundance shown in (C) and (D) normalized to 5xFAD animals. Bars represent mean C83/C99 ratio with individual data points. 


\subsection{Does lack of neuronal cholesterol synthesis affect $A \beta$ production and deposition?}

Reduction in neuronal cholesterol content diminished $A \beta$ production in vitro (Simons et al., 1998; Wahrle et al., 2002), but the role of neuronal cholesterol synthesis in $\mathrm{A} \beta$ generation in vivo is largely unknown. Inactivation of cholesterol synthesis in all neural cells or embryonically in forebrain projection neurons led to neonatal death of mutant mice (Saito et al., 2009; Fünfschilling et al., 2012). However, postnatal loss of cholesterol biosynthesis in forebrain projection neurons is fully compensated by increased synthesis and enhanced transfer of cholesterol via ApoE lipoproteins by other cells including astrocytes (Fünfschilling et al., 2012). In these mice, hereafter termed neuroSQS mutants, recombination in the majority of projection neurons (Fünfschilling et al., 2012) including cortex and hippocampus is driven by the CaMKlla-Cre line (Minichiello et al., 1999) starting from postnatal day 5 (P5, Brinkmann et al., 2008). The lack of CNS pathology and the targeting of neurons in regions of initial $A \beta$ deposition in 5xFAD mice (Oakley et al., 2006) render neuroSQS mice a suitable model to investigate the role of neuronal cholesterol synthesis during $A D$ pathology. Conditional mutants with the genotype CaMKIlaCre ${ }^{\star} 5 x F A D^{*}$ Fdft $1^{\text {floxflox}}$, hereafter termed neuroSQS-5xFAD mutants, and controls were analyzed at P70 (Figure $19 \mathrm{~A}$ ) because preliminary experiments indicated an early effect in $A \beta$ deposition (data not shown). Conditional neuroSQS-5xFAD mice were healthy despite slightly reduced body weight in male mice (Figure $19 \mathrm{~B}$ ) and lacked any obvious behavioral abnormalities. 


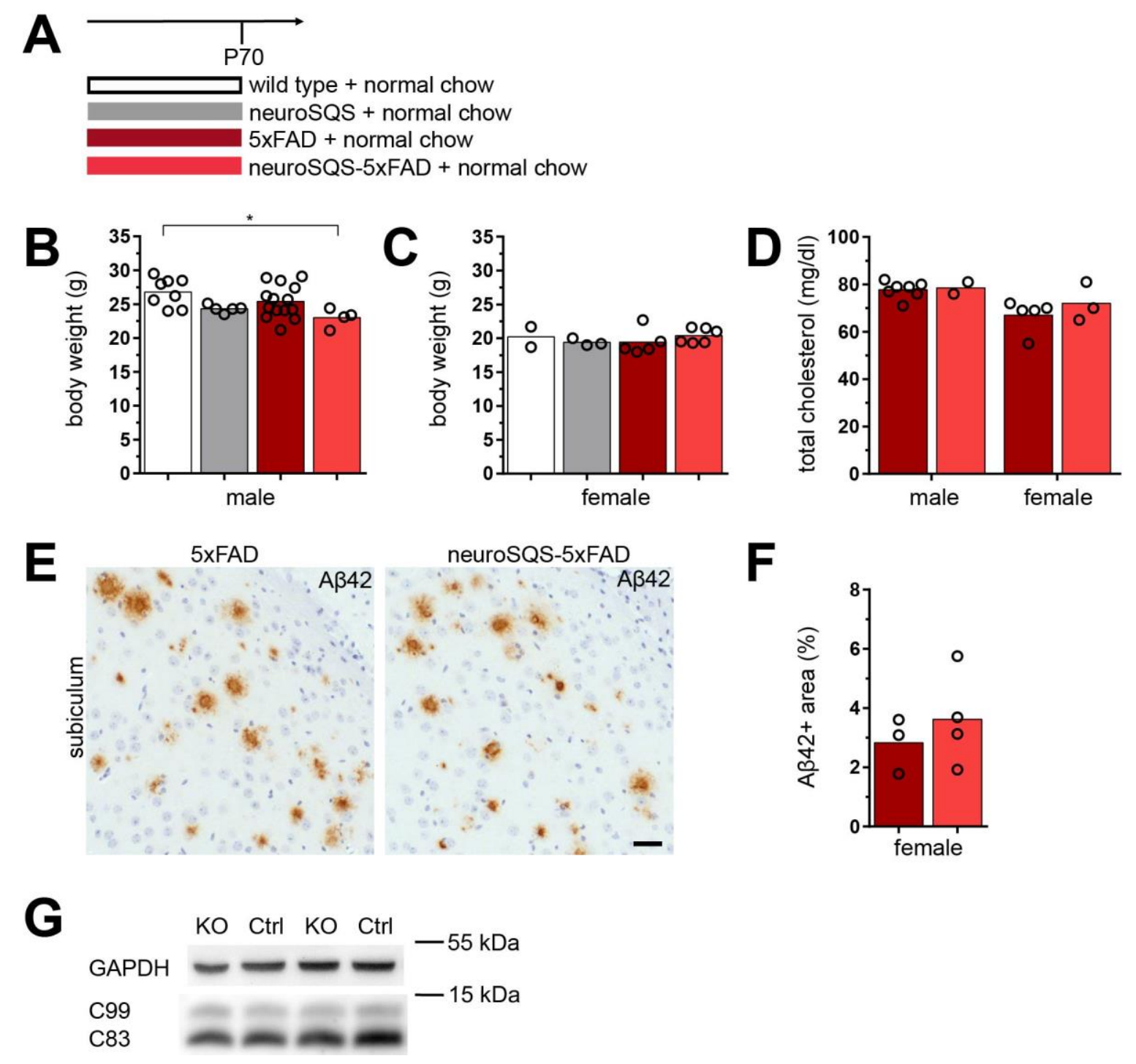

Figure 19: Phenotypic assessment of compound neuroSQS-5XFAD mutants. A) Scheme depicting the time point of analysis of wild type, neuroSQS as well as 5xFAD and compound neuroSQS-5XFAD mutants receiving normal chow. Male and female mice were analyzed separately. Body weight (g) of male (B) and female (C) mice. Bars represent mean values with individual data points (wild type: male, $n=8$, female, $n=2$; neuroSQS: male, $n=$ 5 , female, $n=3$; 5xFAD: male, $n=14$, female, $n=5$; neuroSQS-5xFAD: male, $n=4$, female, $\mathrm{n}=6$ ). D) Total serum cholesterol concentration $(\mathrm{mg} / \mathrm{dl})$ of male and female $5 \times F A D$ and neuroSQS-5XFAD mice. Bars represent mean values with individual data points (5xFAD: male, $n=7$, female, $n=5$; neuroSQS-5xFAD: male, $n=2$, female, $n=3$ ). E) $A \beta 42$ immunolabeling in the subiculum of female 5XFAD and neuroSQS-5XFAD animals. Scale, $40 \mu \mathrm{m}$. F) Quantification of $A \beta 42$ deposition in the subiculum of female $5 \times F A D$ and neuroSQS5XFAD animals. Bars represent mean $A \beta 42$ positive area (\%) with individual data points (5xFAD, $n=3$, astroSQS-5xFAD, $n=4)$. G) Western blot analysis of APP C-terminal fragments C99 and C83 of dissected hippocampi (SDS-soluble protein fraction) of male 5xFAD and neuroSQS-5xFAD animals. KO, knock-out (neuroSQS-5xFAD); Ctrl, control (5xFAD); $\mathrm{kDa}$, kilodalton. Asterisks represent significant differences with ${ }^{*} \mathrm{p}<0.05$ (one-way ANOVA with Tukey's multiple comparison test). 
Serum cholesterol measurements suggested no changes in total cholesterol concentration in male and female mice although the number of animals investigated was low (Figure $19 \mathrm{D}$ ). Quantification of A 342 deposition in the subiculum revealed no difference between female 5XFAD and neuroSQS-5xFAD mice (Figure $19 \mathrm{~F}$ ). In order to assess the effect of neuronal loss of SQS on APP processing, the abundance of APP C-terminal fragments C99 and C83 was analyzed in the hippocampus by western blot. Analysis of two male (Figure $19 \mathrm{G}$ ) and two female (data not shown) neuroSQS-5xFAD mice and two corresponding 5xFAD controls did not suggest major changes in APP processing. So far, loss of neuronal cholesterol synthesis does not seem to induce major changes in the generation and deposition of $A \beta$ peptides. Future experiments will show whether, similar to astroSQS-5xFAD mutants, cholesterol manipulation affects $A \beta$ deposition in male neuroSQS-5XFAD mice that exhibit ameliorated disease compared to female animals. 


\section{Discussion}

\subsection{Efficient targeting of astrocytes in Aldh1l1-CreERT2 mice}

Analysis of gene function in adult astrocytes has been limited by the lack of transgenic mouse lines that express inducible Cre recombinase in the majority of cortical and spinal cord astrocytes. The first aim of this study was to assess efficiency and specificity of CreERT2 mediated recombination in a newly generated Aldh1l1-CreERT2 BAC transgenic mouse line (Winchenbach et al., 2016). Analysis of CreERT2 mediated tdTomato reporter expression revealed efficient targeting of astrocytes throughout the adult brain and in the spinal cord. Quantification using the astrocyte marker S100beta revealed that the vast majority $(>90 \%)$ of cortical astrocytes are targeted. Efficiency of reporter expression in the cortex was confirmed in mice that also express Egfp mediated by the Aldh111-Egfp BAC transgene (Heintz, 2004). Furthermore, the majority of ACSA2 positive astrocytes expressed the Cre reporter tdTomato in isolated astrocytes analyzed by FACS. In our hands, quantification of CreERT2 mediated recombination using a different reporter line (Srinivas et al., 2001) showed that only two thirds of cortical astrocytes were recombined indicating that the tdTomato reporter is more sensitive. Recently, the same tdTomato reporter line (Madisen et al., 2010) was used to demonstrate recombination of astrocytes in Slc1a3-CreERT2 (Mori et al., 2006) and GFAPCreERT2 (Hirrlinger et al., 2006) mice (Jahn et al., 2015). The presented data suggest tdTomato recombination in the minority of cortical astrocytes in GFAPCreERT2 animals while tdTomato expression appeared patchy in the cortex of Slc1a3-CreERT2 animals. Together, these data show that cortical astrocytes are efficiently targeted in adult Aldh111-CreERT2 mice, which had not been possible with previously generated lines (Hirlinger et al., 2006; Mori et al., 2006; Slezak et al., 2007). The results of the current study are in agreement with data reported by an independent group using a different Aldh111-CreERT2 mouse line (Srinivasan et al., 2016).

In Aldh1l1-CreERT2 animals, reporter recombination was virtually absent in neurons and microglia. However, tdTomato expression was found in a subset of cells expressing the oligodendrocyte lineage marker Olig2 and in some NG2 expressing oligodendrocyte precursor cells (OPC). However, the Cre reporter was 
virtually absent in CAll positive mature oligodendrocytes 12 days and 27 weeks after tamoxifen administration. This indicates that the few tdTomato expressing OPC do not give rise to mature oligodendrocytes in adult animals. Future experiments are required to assess whether this is also the case when recombination is induced before maximal oligodendrocyte differentiation during myelination. In the Aldh1l1CreERT2 line generated by Srinivasan et al., transcriptome profiling revealed expression of Olig1, Olig2, CNP and CAll but not NG2 in targeted cortical astrocytes (Srinivasan et al., 2016). Whether these differences are due to the different experimental approaches employed remains to be determined. In summary, the newly generated Aldh1l1-CreERT2 mouse line characterized in the present study is a valuable tool to target astrocytes at desired time points.

\subsection{Inactivation of cholesterol synthesis in adult astrocytes}

The role of astrocytic cholesterol synthesis in brain cholesterol metabolism and function in vivo is incompletely understood. Targeting of lipid and cholesterol synthesis in astrocytes already during development has led to detrimental effects in brain development. Genetic inactivation of the sterol sensor SCAP in astrocytes resulted in severe brain defects including microcephaly and reduction of cholesterol and fatty acid synthesis leading to premature death (Camargo et al., 2012). Mutant mice lacking Srebf2, a transcriptional regulator of cholesterol and lipid synthesis, in astrocytes survived but showed microcephaly and impaired memory and motor defects (Ferris et al., 2017). To address the role of astrocytic cholesterol in adult animals, we inactivated cholesterol biosynthesis by crossing SQS-flox mice (Saher et al., 2005) with the newly generated Aldh111-CreERT2 mouse line followed by tamoxifen administration at 8 weeks of age. We found that peripheral cholesterol supply in the chow was required to rescue dysregulation of serum cholesterol and weight loss, likely attributed to transgene activity in the liver (Winchenbach et al., 2016). Mutant mice on cholesterol chow were healthy and lacked any obvious behavioral abnormalities. Astrocyte density and morphology was unchanged in the cortex of conditional mutants and there were no signs of microgliosis suggesting that mutant astrocytes do not undergo apoptosis. Surprisingly, albeit unchanged astrocyte density the number of Tdtomato expressing astrocytes was reduced in 
mutant mice. Assessment of recombination efficiency of the ROSA26 and Fdft 1 loci suggested that presence of the tdTomato allele reduced Fdft1 recombination. It is possible, that in turn also recombination of the ROSA26 locus was affected leading to reduced tdTomato expression.

In mutant mice the expression of Hmgcr and Fdft1 was unchanged compared to controls while the expression of Apoe was slightly but significantly increased. Significantly reduced Fdft 1 expression in astrocytes isolated from mutant mice without the tdTomato reporter indicated successful ablation of Fdft1 in the majority of cortical astrocytes. Remaining Fdft1 expression might be explained by differences in targeted cell subpopulations by Aldh111-CreERT2 and the ACSA2 antibody (see FACS data) used for magnetic cell isolation. These data suggest that cholesterol synthesis in the cortex is not globally impaired and it is possible that other cell types increased cholesterol synthesis and transport to compensate for the loss of astrocytic cholesterol synthesis as indicated in mice lacking cholesterol synthesis in myelinating glia or neurons (Saher et al., 2005; Fünfschilling et al., 2012). Future experiments are required to assess whether these expressional changes also translate to altered protein abundance.

Immunohistochemical assessment indicated that mutant astrocytes survive but to ensure that these cells were viable, we analyzed them electrophysiologically. Mutant astrocytes exhibited electrophysiological properties indistinguishable from mature wild type cells, indicating that astrocytes are viable in conditional mutants and are not newly generated. Astrocytes are involved in maintaining BBB integrity via ApoE (Bell et al., 2012). We found that BBB integrity is preserved in conditional mutants preventing entry of Evans Blue from the blood circulation. Mutant animals also lacked edema formation as indicated by unchanged brain water content and normal Aqp4 protein levels. A slight increase of bodipy-cholesterol in the brains of mutant mice indicated that peripheral cholesterol might enter the brain at low levels. Investigation of the localization of this cholesterol derivate in the brain could promote the understanding whether peripheral cholesterol influences brain cholesterol metabolism in these mice. In summary, inactivation of cholesterol synthesis in adult astrocytes does not seem to affect astrocyte viability and survival as well as the ability of astrocytes to maintain BBB integrity. 


\subsection{Role of astrocytic cholesterol synthesis in cerebral $\beta$-amyloidosis}

The importance of cholesterol metabolism in Alzheimer's disease is highlighted by the fact that 3 of the top 5 genetic risk factors for AD are involved in cholesterol metabolism (Bertram et al., 2007). Accumulating evidence from in vitro and preclinical studies indicate that cholesterol influences the generation and deposition of $A \beta$ (Maulik et al., 2013). However, it is unclear how cholesterol synthesis by different cell types influences cerebral $\beta$-amyloidosis in vivo. To address this, we have inactivated SQS in astrocytes of 5xFAD mice (Oakley et al., 2006), an animal model of $A D$. Male astroSQS-5XFAD mice showed slightly reduced $A \beta$ immunoreactivity (pan $A \beta$ antibody) throughout the hippocampus compared to controls without reaching statistical significance. Similarly, soluble $A \beta 40$ and $A \beta 42$ species as assessed by an electrochemiluminescence-linked immunoassay in the hippocampus appeared unaltered in astroSQS-5xFAD mice. In 5xFAD mice, A $\beta$ deposition starts in the subiculum of the hippocampus at around two months of age and $A \beta 42$ is found at higher levels than $A \beta 40$ in the brains of $5 x F A D$ mice (Oakley et al., 2006). Indeed, we found significantly reduced $A \beta 42$ deposition in the subiculum of astroSQS-5xFAD mice. Future experiments will reveal whether this holds true during advanced disease in female animals and in aged animals. We next investigated possible explanations for the reduction of $A \beta$ deposition in astroSQS5xFAD animals.

Neuroinflammation with activation of astrocytes and microglia is a common feature in the brains of $A D$ patients and mouse models of $A D$ and both cell types are able to degrade $A \beta$ (Solito and Sastre, 2012; Ferrer, 2017). ApoE was found to facilitate the degradation of $A \beta$ by astrocytes and microglia in vitro (Koistinaho et al., 2004; Jiang et al., 2008). Furthermore, promoting lipidation of $A p o E$ increased $A \beta$ clearance by microglia in vitro and reduced $A \beta$ deposition in vivo (Jiang et al., 2008). It is possible that loss of cholesterol synthesis in astrocytes could affect $A \beta$ clearance by astrocytes and microglia. To address this, we investigated astrocyte and microglia responses in astroSQS-5xFAD mice. Immunohistochemical quantification and expression analysis did not reveal differences in the activation of astrocytes and microglia in astroSQS-5xFAD animals compared to 5xFAD controls. Also the expression of $A \beta$ degrading enzymes was unaltered in the hippocampus of astroSQS-5xFAD mice compared to 5xFAD controls. So far, we have not addressed 
regulation of these enzymes cell type specifically. Interestingly, the expression of Trem2 was significantly upregulated in the hippocampus of astroSQS-5xFAD animals compared to controls. Genetic variants of Trem2 have been identified that increase the risk of $A D$ (reviewed in Ulrich et al., 2017). In mice, Trem2 is highly expressed in microglia cells (Hickman et al., 2013; Zhang et al., 2014) and Trem2 has been shown to associate with various lipids (Wang et al., 2015) and binds ApoE (Atagi et al., 2015; Bailey et al., 2015; Yeh et al., 2016) and ApoJ in vitro (Yeh et al., 2016). Furthermore, association of $A \beta$ with lipoprotein particles enhanced uptake of A $\beta$ by wild type microglia which was impaired in Trem2 deficient microglia (Yeh et al., 2016). In vivo, activation of Trem2 signaling was found in disease-associated microglia in 5xFAD mice (Keren-Shaul et al., 2017). Trem2 deficiency in 5xFAD mice resulted in reduction of plaque associated microglia (Wang et al., 2015; 2016) and increased $A \beta$ deposition (Wang et al., 2015). Together, Trem2 seems to be involved in uptake of lipoprotein associated $A \beta$ and reduced Trem2 expression could hinder $A \beta$ clearance by microglia. It is therefore possible that increased expression of Trem2 by microglia could influence $A \beta$ clearance in astroSQS-5xFAD mice. However, further studies are required to investigate this issue in more detail.

In astroSQS mice, loss of astrocytic cholesterol synthesis did not lead to global changes in cholesterol homeostasis in the adult cortex and in the hippocampus. This could indicate that cholesterol demand in the brain is met by compensatory increase of cholesterol synthesis and transport by other cell types as reported in mice that lacked cholesterol synthesis in myelinating glia or forebrain projection neurons (Saher et al., 2005; Fünfschilling et al., 2012). Similarly, lipid transport could also be enhanced in astroSQS-5XFAD mice. Lipid transport within the brain likely influences the distribution and deposition of $A \beta$ (Holtzman et al., 2012). Decreased ApoE lipidation (Hirsch-Reinshagen et al., 2004) resulted in increased $A \beta$ deposition in mice lacking Abca1 (Koldamova et al., 2005; Wahrle et al., 2005) while overexpression of $A b c a 1$ resulted in decreased $A \beta$ deposition (Wahrle et al., 2008). Therefore, in the next step we investigated cholesterol homeostasis in the hippocampus of 5XFAD and astroSQS-5xFAD animals by expressional analysis of the major genes involved in cholesterol synthesis, transport, uptake and excretion. Expression analyses revealed no differences in astroSQS-5xFAD animals compared to $5 \times$ FAD mice, suggesting that similar to wild type mice, tissue cholesterol homeostasis is not altered by loss of astrocytic cholesterol synthesis in 
5xFAD animals. Surprisingly, ApoE protein levels were also unaltered in astroSQS and astroSQS-5xFAD compared to controls. Further study is required to assess whether microglia and neurons synthesize cholesterol when Fdft1 expression is inactivated in astrocytes, and which cell types generate ApoE. Whether lipid transport between cell types contributes to the reduction in $A \beta$ deposition in astroSQS-5XFAD mice remains an open question.

As discussed above, tissue cholesterol homeostasis seemed to be unaltered in astroSQS-5xFAD mutants. However, it remains unclear which cell types synthesize cholesterol in the absence of astrocytic cholesterol synthesis. Also, it is not well understood to which extent neurons require cholesterol supply by astrocytes in vivo. In vitro, cholesterol supported the clustering and endocytosis of APP and Bace1 (Marquer et al., 2011), while reducing neuronal cholesterol content diminished $A \beta$ generation (Simons et al., 1998; Wahrle et al., 2002). We therefore asked, whether loss of astrocytic cholesterol supply would in turn affect neuronal cholesterol content and thereby modulate APP processing to generate A . To address this, APP Cterminal fragments resulting from $\alpha$-secretase cleavage (C83) and from amyloidogenic Bace1 processing (C99) were quantified in the hippocampus of astroSQS-5XFAD animals. However, we did not find a difference in the ratio of C83/C99 fragments in astroSQS-5XFAD and 5XFAD mice indicating that neuronal APP processing by $\alpha$-secretase and Bace 1 is not altered in astroSQS-5xFAD animals. It could be possible that neurons acquire cholesterol from other cells than astrocytes or synthesize enough cholesterol themselves. Further research is required to address these questions.

Surprisingly, expression of the Bace1 homologue Bace2 was reduced in 5xFAD animals compared to astroSQS-5xFAD mice. In vitro, Bace2 cleaves APP at the $\beta$ site (Hussain et al., 2000) but more prominently near the site of $\alpha$-secretase cleavage (Yan et al., 2001). Overexpression of Bace2 reduced $A \beta$ production in vitro and Bace2 could act as an $A \beta$ degrading enzyme (Sun et al., 2006; Abdul-Hay et al., 2012). However, the role of Bace2 in vivo is far from understood and further research is required to assess whether Bace2 mediated APP processing could affect $A \beta$ generation and deposition. 
In summary, inactivation of cholesterol synthesis in astrocytes leads to a reduction in AB42 deposition in the subiculum of astroSQS-5xFAD mice. There, increased expression of Trem2 could potentially facilitate $A \beta$ clearance by microglia cells. In mutant mice cholesterol homeostasis is largely unaltered, but it remains unclear which cell types compensate for the lack of astrocytic cholesterol synthesis. Loss of cholesterol support by astrocytes did not affect neuronal APP processing raising the question whether cell autonomous cholesterol synthesis is required in neurons to generate $A \beta$.

\subsection{Role of neuronal cholesterol synthesis in cerebral $\beta$-amyloidosis}

In neuroSQS animals, loss of cholesterol synthesis in forebrain projection neurons if fully compensated by increased synthesis and transport of cholesterol by other cell types, likely astrocytes (Fünfschilling et al., 2012). The extent of cell autonomous cholesterol synthesis by neurons however is incompletely understood. Furthermore, it is unclear whether external supply of cholesterol by other cells or neuronal cholesterol synthesis itself affects APP processing and A $\beta$ generation. In astroSQS-5XFAD animals, neuronal APP processing was not altered leading to the question whether neuronal cholesterol synthesis influences APP processing and the deposition of $A \beta$. To address this question we inactivated cholesterol synthesis in neurons of 5XFAD mice. In these neuroSQS-5xFAD mutants A 42 deposition was unchanged compared to $5 \times \mathrm{FAD}$ controls and quantification of APP C-terminal fragments in few animals did not suggest major changes in APP processing. However, these data have to be verified using increased animal numbers. In conclusion, the analysis of astroSQS-5xFAD and neuroSQS-5xFAD animals in parallel could provide valuable insight into the role of cellular cholesterol synthesis of different cell types and tissue cholesterol homeostasis in the generation and deposition of $A \beta$. 


\section{Material}

\subsection{Solutions and buffers}

\section{Avertin}

2 g 2,2,2 tribromoethanol 99\% (T48402, Sigma)

$2 \mathrm{ml}$ tert-Amyl alcohol (Sigma)

$96 \mathrm{ml} \mathrm{H}{ }_{2} \mathrm{O}\left(40^{\circ} \mathrm{C}\right)$

Avertin was stirred for $30 \mathrm{~min}$ and was sterile filtered. The solution was stored at $-20^{\circ} \mathrm{C}$ and heated to $37^{\circ} \mathrm{C}$ before usage.

\section{TBE buffer 20x (stock solution)}

1.8 M Trisbase

1.8 $\mathrm{M}$ boric acid

200 mM EDTA (ethylenediaminetetraacetic acid)

Diluted 1:20 with $\mathrm{ddH}_{2} \mathrm{O}$ before use (1x TBE buffer).

\section{Sucrose buffer pH 7.4 (lysate preparation)}

(modified from Saab et al., 2016)

$320 \mathrm{mM}$ sucrose

$10 \mathrm{mM}$ Trisbase

$2 \mathrm{mM} \mathrm{NaHCO} 3$

$1 \mathrm{mM} \mathrm{MgCl} 2$

cOmplete ${ }^{\mathrm{TM}}$ protease inhibitors (Roche)

PhosSTOPTM phosphatase inhibitors (Roche)

\section{TBS buffer pH 8 (lysate preparation)}

$120 \mathrm{mM} \mathrm{NaCl}$

$50 \mathrm{mM}$ Trisbase

cOmplete $^{\mathrm{TM}}$ protease inhibitors (Roche)

SDS buffer (Iysate preparation)

$2 \%(w / v)$ sodium dodecyl sulfate (SDS)

cOmplete ${ }^{\mathrm{TM}}$ protease inhibitors (Roche) 


\section{4x SDS buffer pH 6.8}

$40 \%(v / v)$ glycerol

$240 \mathrm{mM}$ Trisbase

$8 \%(v / v)$ SDS

0.04\% (w/v) bromophenol blue

\section{X reducing sample buffer $\mathrm{pH} 7$}

(modified from Schägger, 2006)

$150 \mathrm{mM}$ Trisbase

$12 \%(w / v)$ SDS

$6 \%(\mathrm{v} / \mathrm{v})$ dithiothreitol (500 mM)

$30 \%(w / v)$ glycerol

$0.05 \%$ Coomassie blue

10x running buffer (Laemmli buffer)

$250 \mathrm{mM}$ Trisbase

$1.92 \mathrm{M}$ glycine

$1 \%(\mathrm{v} / \mathrm{v}) \mathrm{SDS}$

diluted 1:10 with $\mathrm{dd}_{2} \mathrm{O}$ before use

1x MES buffer pH 7.3

50 mM MES

$50 \mathrm{mM}$ Trisbase

$0.1 \%(v / v)$ SDS

$1 \mathrm{mM}$ EDTA

10x transfer buffer

$480 \mathrm{mM}$ Trisbase

$390 \mathrm{mM}$ glycine

$10 \%(\mathrm{v} / \mathrm{v})$ ethanol

diluted 1:10 with $\mathrm{dd}_{2} \mathrm{O}$ before use

\section{1x TBS-T pH 7.5}

$50 \mathrm{mM}$ Trisbase

$150 \mathrm{mM} \mathrm{NaCl}$

0.05\% (v/v) Tween-20 


\section{Blocking buffer}

$5 \%(\mathrm{w} / \mathrm{v})$ non-fat dry milk powder in $1 \mathrm{x}$ TBST

Tissue storage solution (FACS)

$2252 \mu \mathrm{l} 1 \mathrm{M}$ glucose in $1 \mathrm{x}$ PBS

$2597 \mu \mathrm{l} 1 \mathrm{M} \mathrm{NaHCO} 3$ in 1x PBS

Adjust $\mathrm{pH}$ to 7.3 with $1 \mathrm{M} \mathrm{NaOH}$.

$2000 \mathrm{ml}$ DNAse $(10 \mathrm{mg} / \mathrm{ml})$

EDTA solution (FACS)

50 mM EDTA

$100 \mathrm{mM}$ L-cystein

in $\mathrm{dd}_{2} \mathrm{O}$.

\section{Digest solution (FACS)}

$2.5 \mathrm{ml}$ tissue storage solution

$25 \mu$ I EDTA solution

$50 \mu \mathrm{l}$ Papain (Worthington, LS003126)

Inhibitor solution (FACS)

$50 \mathrm{ml} \mathrm{10 \%} \mathrm{HEPES} \mathrm{in} \mathrm{1x} \mathrm{PBS}$

$450 \mathrm{ml}$ MEM (Gibco)

Adjust $\mathrm{pH}$ to 7.3 with $1 \mathrm{M} \mathrm{NaOH}$.

$11.261 \mathrm{ml} 1 \mathrm{M}$ glucose in $1 \times$ PBS

$12.987 \mathrm{ml} 1 \mathrm{M} \mathrm{NaHCO} 3$ in $1 \times$ PBS

$500 \mathrm{mg}$ bovine serum albumin

\section{FACS buffer}

$2 \%$ bovine serum albumin $(\mathrm{v} / \mathrm{v})$ in $1 \mathrm{xPBS}$

10x PBS (histology)

$1.7 \mathrm{M} \mathrm{NaCl}$

$0.04 \mathrm{M} \mathrm{KCl}$

$0.04 \mathrm{M} \mathrm{Na}_{2} \mathrm{HPO}_{4}$

$0.018 \mathrm{M} \mathrm{KH}_{2} \mathrm{PO}_{4}$

Adjusted to $\mathrm{pH} 7.2$ with $1 \mathrm{M} \mathrm{NaOH}$ and diluted 1:10 with $\mathrm{ddH}_{2} \mathrm{O}$ (1x PBS) 
$16 \%$ (w/v) Paraformaldehye stock solution

$16 \%(\mathrm{w} / \mathrm{v})$ paraformaldehyde cooked at $65^{\circ} \mathrm{C}$ for $15 \mathrm{~min}$ while stirring

Add $5 \mathrm{~N} \mathrm{NaOH}$ until solution turns clear, filter and store at $-20 \mathrm{C}^{\circ}$.

\subsection{Phosphate buffer (histology)}

$0.36 \%(\mathrm{w} / \mathrm{v})$ Sodiumdihydrogenphosphate $\left(\mathrm{NaH}_{2} \mathrm{PO}_{4}\right)$

$3.1 \%(w / v)$ di-Sodiumhydrogenphosphate $\left(\mathrm{Na}_{2} \mathrm{HPO}_{4}\right)$

$1 \%(\mathrm{w} / \mathrm{v}) \mathrm{NaCl}$

4\% (v/v) Paraformaldehye (PFA) for histology

$4 \%(\mathrm{v} / \mathrm{v})$ paraformaldehyde

$0.1 \mathrm{M}$ Phosphate buffer

\section{Anti-freeze solution}

$300 \mathrm{ml}$ glycerol

$300 \mathrm{ml}$ ethylene glycol

$400 \mathrm{ml} 1 \mathrm{x}$ PBS

\section{$0.01 \mathrm{M}$ sodium citrate buffer $\mathrm{pH} 6$}

$1.8 \mathrm{mM}$ citric acid

$8.2 \mathrm{mM}$ sodium citrate

Prepared freshly before use.

Tris buffer pH 7.6 (histology)

50 mM Trisbase

$0.9 \%(\mathrm{w} / \mathrm{v}) \mathrm{NaCl}$

Prepared freshly before usage.

\subsection{Antibodies}

Table 3: Primary antibodies

\begin{tabular}{|l|l|l|l|l|l|}
\hline Antibody & Species & Cat. No. & Company & Dilution & Purpose \\
\hline APP & poly-rabbit & A8717 & Sigma-Aldrich & $1: 2000$ & WB \\
\hline CAIl & poly-rabbit & n.a. & $\begin{array}{l}\text { M. Said } \\
\text { Ghandour }\end{array}$ & $1: 100$ & IHC \\
\hline GAPDH & mono-mouse & CSA-335 & Stressgen & $1: 2500$ & WB \\
\hline GFAP & mono-mouse & MAB3402 & Chemicon & $1: 200$ & IHC \\
\hline GFP & poly-goat & $600-101-215$ & Rockland & $1: 500$ & IHC \\
\hline
\end{tabular}




\begin{tabular}{|l|l|l|l|l|l|}
\hline Iba1 & poly-rabbit & $019-19741$ & Wako & $1: 1000$ & IHC \\
\hline Mac-3 & mono-rat & 553322 & $\begin{array}{l}\text { BD } \\
\text { Pharmingen }\end{array}$ & $1: 500$ & IHC \\
\hline NSE & poly-rabbit & AB951 & Chemicon & $1: 500$ & IHC \\
\hline Olig2 & poly-rabbit & DF308 & $\begin{array}{l}\text { Charles Stiles, } \\
\text { John Alberta }\end{array}$ & $1: 100$ & IHC \\
\hline Parvalbumin & poly-rabbit & PV-28 & Swant & $1: 1000$ & IHC \\
\hline RFP & poly-rabbit & $600-401-379$ & Rockland & $1: 500 /$ & IHC / WB \\
\hline S100beta & mono-rabbit & ab52642 & Abcam & $1: 200$ & IHC \\
\hline A 338/40/42/43 & mono-mouse & $\begin{array}{l}218211 \\
(\mathrm{NT} 244)\end{array}$ & $\begin{array}{l}\text { Synaptic } \\
\text { Systems }\end{array}$ & $1: 500$ & IHC \\
\hline A 342 & poly-rabbit & $44-344$ & $\begin{array}{l}\text { ThermoFisher } \\
\text { Scientific }\end{array}$ & $1: 500$ & IHC \\
\hline Aquaporin4 & poly-rabbit & $\begin{array}{l}\text { sc-20812 } \\
\text { (H-80) }\end{array}$ & Santa Cruz & $1: 500$ & IHC \\
\hline ApoE & poly-goat & AB947 & Chemicon & $1: 2000$ & WB \\
\hline AN2 & poly-rat & n.a. & J. Trotter & $1: 50$ & IHC \\
\hline
\end{tabular}

Table 4: Secondary antibodies

\begin{tabular}{|l|l|l|l|l|l|}
\hline Antibody & Species & Cat. No. & Company & Dilution & Purpose \\
\hline a-goat-Alexa488 & donkey & A-11055 & Invitrogen & $\begin{array}{l}1: 1000 / \\
1: 2000\end{array}$ & IHC \\
\hline $\begin{array}{l}\text { a-rabbit- } \\
\text { DyLight633 }\end{array}$ & donkey & 356 & YO Proteins & $1: 500$ & IHC \\
\hline a-rabbit-Alexa488 & donkey & A-21206 & Invitrogen & $1: 1000 /$ & IHC \\
& & & & $1: 2000$ & \\
\hline a-rabbit-Alexa555 & donkey & A-31572 & Invitrogen & $1: 1000 /$ & IHC \\
& & & & $1: 2000$ & \\
\hline $\begin{array}{l}\text { a-mouse- } \\
\text { Alexa488 }\end{array}$ & donkey & A-21202 & Invitrogen & $1: 1000 /$ & IHC \\
& & & & $1: 2000$ & \\
\hline a-mouse-HRP & goat & $115-035-003$ & Dianova & $1: 5000$ & WB \\
\hline a-goat-HRP & rabbit & A5420 & Sigma & $1: 5000$ & WB \\
\hline a-rabbit-HRP & goat & $111-035-003$ & Dianova & $1: 5000$ & WB \\
\hline
\end{tabular}




\subsection{Primers}

Table 5: Genotyping primers

\begin{tabular}{|c|c|c|}
\hline Target locus & Primer sequence & $\begin{array}{l}\text { In house } \\
\text { Cat.no }\end{array}$ \\
\hline \multirow[t]{2}{*}{ Aldh1l1-CreERT2 } & se: 5'-CAACTCAGTCACCCTGTGCTC-3' & 20713 \\
\hline & as: 5'-TTCTTGCGAACCTCATCACTCG-3' & 08250 \\
\hline \multirow[t]{2}{*}{ Aldh1/1-Egfp } & se: 5'-CCCTTTCTAACCCTCTGGC-3' & 22022 \\
\hline & as: 5'-TCCTTGAAGAAGATGGTGCG 3' & 15526 \\
\hline \multirow[t]{2}{*}{ CaMKIla-Cre } & se: 5'-GGGAGGTAGGAAGAGCGATG-3' & 06722 \\
\hline & as: 5'-CCATGAGTGAACGAACCTGG-3' & 06723 \\
\hline \multirow[t]{3}{*}{ Fdft1-flox } & se: 5'-ACGGGGCCAGGGTGCTTTTCTC-3' & 02884 \\
\hline & as 1: 5'-CTTCCCTTTCCTTGCCTTTCCATA-3' & 32822 \\
\hline & as 2: 5'-GGCTTATCTCTTCTCGCAATCTGA-3' & 02720 \\
\hline \multirow[t]{3}{*}{ NG2-Eyfp } & se: 5'-CTAAGCGCGGGTCTGGCGCC -3' & 33262 \\
\hline & as 1: 5'-CGCTGAACTTGTGGCCGTTTA -3' & 03312 \\
\hline & as 2: 5'-ACAGCTTTCCTTCCAGAC -3' & 05039 \\
\hline \multirow[t]{3}{*}{ Slc1a3-CreERT2 } & se: 5'-GAACTACAATCCTTTAAGGCTCACG -3' & 26605 \\
\hline & as 1: 5'-GCAGGACCTCGGGGTAGTCAC -3' & 26606 \\
\hline & as 2: 5'-CACCAGAGACGGAAATCCATCG -3' & 26607 \\
\hline \multirow{3}{*}{$\begin{array}{l}\text { ROSA26- } \\
\text { Tdtomato }\end{array}$} & se: 5'-CTCTGCTGCCTCCTGGCTTCT -3' & 14025 \\
\hline & as 1: 5'-TCAATGGGCGGGGGTCGTT -3' & 14024 \\
\hline & as 2: 5'-CGAGGCGGATCACAAGCAATA -3' & 14026 \\
\hline \multirow[t]{3}{*}{ ROSA26-Eyfp } & se: 5'-AAAGTCGCTCTGAGTTGTTAT -3' & 03735 \\
\hline & as 1: 5'-GCGAAGAGTTTGTCCTCAACC -3' & 03736 \\
\hline & as 2: 5'-GGAGCGGGAGAAATGGATATG -3' & 03737 \\
\hline \multirow[t]{2}{*}{$5 x F A D$} & se: 5'-CCGCGCAGAACAGAAGGACAGAC -3' & 19746 \\
\hline & as: 5'-GGGCGGGCATCAACAGGCTCAACT -3' & 19747 \\
\hline
\end{tabular}


Table 6: Quantitative RT-PCR primers

\begin{tabular}{|c|c|c|}
\hline Target locus & Primer sequence & $\begin{array}{l}\text { In house } \\
\text { Cat. no }\end{array}$ \\
\hline \multirow[t]{2}{*}{ Abca1 } & se: 5‘-CTGTTTCCCCCAACTTCTG-3، & 19854 \\
\hline & as: 5'-TCTGCTCCATCTCTGCTTTC-3' & 19855 \\
\hline \multirow[t]{2}{*}{ Apoe } & se: 5'-GACCCTGGAGGCTAAGGACT -3' & 11856 \\
\hline & as: 5'-AGAGCCTTCATCTTCGCAAT -3' & 11857 \\
\hline \multirow[t]{2}{*}{ Apoj/ Clusterin } & se: 5'-GCCATGGATGTCCAGCTC -3' & 36475 \\
\hline & as: 5'-CACACAGTGCGGTCATCTTC -3' & 36476 \\
\hline \multirow[t]{2}{*}{ Aqp4 } & se: 5'-TGGAGGATTGGGAGTCACC -3' & 30574 \\
\hline & as: 5'-TGAACACCAACTGGAAAGTGA -3' & 30575 \\
\hline \multirow[t]{2}{*}{ App (human) } & $\begin{array}{l}\text { se: 5'-CCGCTCTGCAGGCTGTTC -3' } \\
\text { (McAlpine et al., 2009) }\end{array}$ & 37065 \\
\hline & $\begin{array}{l}\text { as: 5'-CGCGGACATACTTCTTTAGCATATT -3' } \\
\text { (McAlpine et al., 2009) }\end{array}$ & 37066 \\
\hline \multirow[t]{2}{*}{ App (mouse) } & $\begin{array}{l}\text { se: 5'-TCAGGATTTGAAGTCCGCCA -3' } \\
\text { (Allué et al., 2016) }\end{array}$ & 37067 \\
\hline & as: 5'-TTTGTTCGAACCCACATCTTCAG -3' & 33760 \\
\hline \multirow[t]{2}{*}{ Bace1 } & se: 5'-CCCTTTCCTGCATCGCTAC -3' & 12015 \\
\hline & as: 5'-TACACACCCTTTCGGAGGTC -3' & 12016 \\
\hline \multirow[t]{2}{*}{ Bace2 } & se: 5'-CCTGAGAGATGAGAATGCCAGT -3' & 32361 \\
\hline & as: 5'-ATCATGGGCTGAATGTAGAGC -3' & 32362 \\
\hline \multirow[t]{2}{*}{ Cd9 } & se: 5'-GATATTCGCCATTGAGATAGCC -3' & 13276 \\
\hline & as: 5'-TGGTAGGTGTCCTTGTAAAACTCC -3' & 13277 \\
\hline \multirow[t]{2}{*}{ Ch25h } & se: 5'-AAGACCTGGGCTGTTCCAG -3' & 35387 \\
\hline & as: 5'-GCCTCCCTTGTCCTTATGGT -3' & 35388 \\
\hline \multirow[t]{2}{*}{ Cyp46a1 } & se: 5'-AACTTTGTCACCTTCTTCATTGC -3' & 34294 \\
\hline & as: 5'-CCATCACTGTGAATGCCAGA -3' & 34295 \\
\hline \multirow[t]{2}{*}{ Ece1 } & se: 5'-GTGCTGGTGACGCTTCTG -3' & 37230 \\
\hline & as: 5'-ACATACCGGAGGCGTTCTT -3' & 37231 \\
\hline \multirow[t]{2}{*}{ Ece2 } & se: 5'-GCTTTGAGACTGCACAGGAGA -3' & 37232 \\
\hline & as: 5'-CACCTCGGAGTGCAGGAC -3' & 37233 \\
\hline
\end{tabular}




\begin{tabular}{|c|c|c|}
\hline Fdft1 & se: 5'-CCAAACAGGACTGGGACAAG-3' & 10843 \\
\hline & as: 5'-GACGAGAAAGGCCAATTCC-3' & 10844 \\
\hline \multirow[t]{2}{*}{ Gfap } & se: 5'-TCAAGAGGAACATCGTGGTAAAGA -3' & 09414 \\
\hline & as: 5'-TGCTCCTGCTTCGAGTCCTT -3' & 09416 \\
\hline \multirow[t]{2}{*}{ Hmgcr } & se: 5'-TGATTGGAGTTGGCACCAT -3' & 11766 \\
\hline & as: 5'-TGGCCAACACTGACATGC -3' & 11767 \\
\hline \multirow[t]{2}{*}{ lba1 / Aif1 } & se: 5'-CCGAGGAGACGTTCAGCTAC -3' & 09704 \\
\hline & as: 5'-TGTTTTTCTCCTCATACATCAGAATC -3' & 09705 \\
\hline \multirow[t]{2}{*}{ Ide } & se: 5'-TTCGATGTTTCCCATGAACA-3' & 37063 \\
\hline & as: 5'-AGGGGCACAGGAAAAACTG-3' & 37064 \\
\hline \multirow[t]{2}{*}{ Lamp2 } & se: 5'-AAGGTGCAACCTTTTAATGTGAC -3' & 32099 \\
\hline & as: 5'-TGTCATCATCCAGCGAACAC -3' & 32100 \\
\hline \multirow{2}{*}{ Ldlr } & se: 5'-GATGGCTATACCTACCCCTCAA -3' & 11887 \\
\hline & as: 5'-TGCTCATGCCACATCGTC -3' & 11888 \\
\hline \multirow[t]{2}{*}{ Lpl } & se: 5'-CTCGCTCTCAGATGCCCTAC -3' & 35391 \\
\hline & as: 5'-AGGCCTGGTTGTGTTGCTT -3' & 35392 \\
\hline \multirow[t]{2}{*}{ Lrp1 } & se: 5'-ACCACCATCGTGGAAAATG -3' & 34314 \\
\hline & as: 5'-GTCCCAGCCACGGTGATA -3' & 34315 \\
\hline \multirow[t]{2}{*}{ Lrp2 } & se: 5'-ATGCAGATGAGCACCACTGT -3' & 35485 \\
\hline & as: 5'-CACAGCCGTTTGTGATGAGA -3' & 35486 \\
\hline \multirow[t]{2}{*}{ Rplp0 } & se: 5'-GATGCCCAGGGAAGACAG -3' & 19908 \\
\hline & as: 5'-ACAATGAAGCATTTTGGATAATCA -3' & 19909 \\
\hline \multirow[t]{2}{*}{ Rps13 } & se: 5'-CGAAAGCACCTTGAGAGGAA -3' & 19912 \\
\hline & as: 5'-TTCCAATTAGGTGGGAGCAC -3' & 19913 \\
\hline
\end{tabular}




\section{Methods}

\subsection{Mice}

All animal studies were performed at the Max Planck Institute of Experimental Medicine in compliance with the animal policies of the Max Planck Institute of Experimental Medicine and were approved by the German Federal State of Lower Saxony. All animals were housed in individually ventilated cages in groups of 3-5 mice per cage and kept in a room with controlled temperature $\left(\sim 23^{\circ} \mathrm{C}\right)$ under $12 \mathrm{~h}$ light/dark cycle. Mice received either normal diet or the same diet supplemented with $0.5 \% \mathrm{w} / \mathrm{w}$ cholesterol (ssniff Spezialdiäten $\mathrm{GmbH}$ ). Food and water were provided ad libitum. Male and female C57BI/6N mice generated at the Max Planck Institute of Experimental Medicine were used to maintain and expand mutant lines and served as controls during analyses where indicated. Assessment of recombination in the newly generated Aldh111-CreERT2 mouse line was performed using mice of both sexes at the age of 7-10 weeks unless otherwise stated. In all further studies male and female mice were analyzed separately at the ages indicated.

\subsubsection{Mouse mutants}

The ROSA26 flox-stop-flox-Tdtomato line (ROSA26-Tdtomato; Madisen et al., 2010) and the ROSA26 flox-stop-flox-Eyfp line (ROSA26-Eyfp; Srinivas et al., 2001) were used as Cre reporters. Furthermore, we used BAC transgenic Aldh1l1-Egfp mice (Heintz, 2004), Slc1a3-CreERT2 mice (Mori et al., 2006), and NG2-Eyfp knock-in mice (Karram et al., 2008). As an Alzheimer's disease model we used 5xFAD mice (Oakley et al., 2006) that were back-crossed to C57BI/6N wildtype animals for at least 5 generations. The generation of conditional Fdft1 mutants is described below. 


\subsubsection{Generation of Aldh111-CreERT2 mice}

Generation of Aldh111-CreERT2 mice was performed before the start of this project by Dr. Gesine Saher at the Max Planck Institute of Experimental Medicine as described elsewhere (Winchenbach et al., 2016). Briefly, a CreERT2 cassette (Sauer, 1994) under control of the Aldh1l1 promoter was inserted into exon 2 of the Aldh1l1 locus on a murine BAC (BAC RP23-7M9) by homologous recombination. After excision of the kanamycin resistance cassette the BAC insert was purified by size exclusion chromatography using a sepharose column and used for pronucleus injection. Three out of five founder mice bred to Cre reporter mice showed expression in brain. Based on the degree of expression in astrocytes and minimal expression in other cell types the Aldh1l1-CreERT2 line 02 was selected for further analyses.

\subsubsection{Generation of conditional Fdft1 mutants}

In order to conditionally inactivate cholesterol biosynthesis in astrocytes we crossed Aldh1l1-CreERT2 mice (Winchenbach et al., 2016) with SQS-flox mice (Saher et al., 2005) to obtain Aldh111-CreERT2*Fdft $1^{\text {floxfflox }}$ mice (termed astroSQS mutants). In these mice the Fdft1 gene encoding squalene synthase, the enzyme catalyzing the first committed step in cholesterol synthesis, is flanked by loxP sites allowing excision of the gene by Cre recombinase. CreERT2-mediated recombination was induced in these animals by tamoxifen administration at desired time points (see below). Mutants lacking cell-autonomous cholesterol synthesis in forebrain projection neurons were generated by crossing the CaMKIla-Cre line (Minichiello et al., 1999) to SQS-flox mice (termed neuroSQS mutants) as described previously (Fünfschilling et al., 2012). 


\subsubsection{Generation of 5xFAD mutants}

AstroSQS and neuroSQS mutants were crossed to 5xFAD mice (Oakley et al., 2006) in order to generate astroSQS-5xFAD and neuroSQS-5xFAD mutants. All mouse breedings were designed to ensure hemizygosity of the offspring for the respective Cre alleles and for the 5xFAD transgene as well as homozygosity of the SQS-flox allele. An overview of the experimental groups (identical for male and female cohorts) is listed below.

\begin{tabular}{|c|c|}
\hline Genotype & Name \\
\hline Aldh1I1-CreERT2*Fdft $1^{\text {floxfflox }}$ & astroSQS \\
\hline Fdft1 $1^{\text {flox/flox }}$ or $\mathrm{C} 57 \mathrm{BI} / 6 \mathrm{~N}$ & SQS-flox or wild type \\
\hline Aldh1l1-CreERT2*Fdft1 ${ }^{\text {flox/flox*} 5 x F A D}$ & astroSQS-5xFAD \\
\hline $5 x F A D^{*} F d f t 1^{\text {flox/flox }}$ & $5 x F A D$ \\
\hline
\end{tabular}

\begin{tabular}{|c|c|}
\hline Genotype & Name \\
\hline CaMKIla-Cre ${ }^{*}$ Fdft $1^{\text {flox/flox }}$ & neuroSQS \\
\hline Fdft $1^{\text {flox/flox }}$ or $\mathrm{C} 57 \mathrm{BI} / 6 \mathrm{~N}$ & SQS-flox or wild type \\
\hline CaMKIla-Cre *Fdft1 ${ }^{\text {floxfllox*} 5 x F A D}$ & neuroSQS-5xFAD \\
\hline $5 x F A D^{*} F d f t 1^{f l o x / f l o x}$ & $5 x F A D$ \\
\hline
\end{tabular}

\subsection{DNA purification and genotyping}

Genomic DNA from dissected cortices of adult mice was purified using the NucleoSpin® Tissue kit (Macherey-Nagel, 740952.250) and from tail or ear biopsies

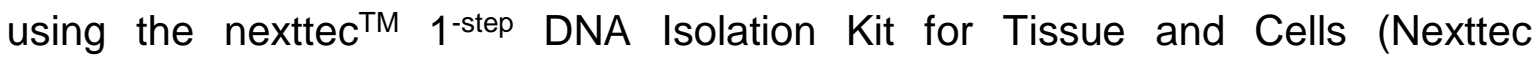
Biotechnology) following the manufacturer's instructions. Subsequently, DNA was either used undiluted or diluted 1:5 in $\mathrm{dd}_{2} \mathrm{O}$ for genotyping by polymerase chain reaction (PCR, Thermocycler T3, Biometra) using GoTaq ${ }^{\circledR}$ polymerase and buffer (Promega, M784B and M791A) according to the protocols listed below. Finally, PCR products were supplemented with $5 \mu \mathrm{l} \mathrm{GelRed}{ }^{\mathrm{TM}}$ (1:4000 in $\mathrm{ddH}_{2} \mathrm{O}$, Biotium, 41003) and loaded on $2 \%$ agarose gels. In order to estimate the size of amplified DNA fragments the GeneRuler 100 bp DNA Ladder (ThermoFisher Scientific, SM0241) 
supplemented with GelRed ${ }^{\mathrm{TM}}$ was loaded as well. Gel electrophoresis was performed in a running chamber filled with $1 \mathrm{x}$ TBE buffer for 60 to $90 \mathrm{~min}$ at $120 \mathrm{~V}$. Pictures were taken under UV light using the Intas UV System (Intas Science Imaging Instruments $\mathrm{GmbH}$ ).

\begin{tabular}{|c|c|}
\hline \multicolumn{2}{|c|}{ Aldh1l1-CreERT2 } \\
\hline \multicolumn{2}{|c|}{ Master mix: } \\
\hline $1 \mu \mathrm{l}$ & DNA \\
\hline $0.5 \mu \mathrm{l}$ & se-primer \#20713 (10 pmol/ $\mu \mathrm{l})$ \\
\hline $0.5 \mu \mathrm{l}$ & as-primer \#8250 (10 pmol/ $/ \mathrm{l})$ \\
\hline $2 \mu \mathrm{l}$ & dNTP (2 mM) \\
\hline $4 \mu \mathrm{l}$ & GoTaq buffer (5x) \\
\hline $0.1 \mu l$ & GoTaq \\
\hline $11.9 \mu \mathrm{l}$ & $\mathrm{dd} \mathrm{H}_{2} \mathrm{O}$ \\
\hline \multicolumn{2}{|c|}{ PCR program: } \\
\hline $1.95^{\circ} \mathrm{C}$ & $3 \mathrm{~min}$ \\
\hline $2.56^{\circ} \mathrm{C}$ & $30 \mathrm{~s}$ \\
\hline 3. $72^{\circ} \mathrm{C}$ & $1 \mathrm{~min}$ \\
\hline 4. $95^{\circ} \mathrm{C}$ & $30 \mathrm{~s}$ \\
\hline \multicolumn{2}{|c|}{$\rightarrow$ Step 2 to 4 for 36 cycles } \\
\hline $5.56^{\circ} \mathrm{C}$ & $1 \mathrm{~min}$ \\
\hline $6.72^{\circ} \mathrm{C}$ & $10 \mathrm{~min}$ \\
\hline 7. $4^{\circ} \mathrm{C}$ & pause \\
\hline
\end{tabular}

\begin{tabular}{|c|c|}
\hline \multicolumn{2}{|c|}{ Aldh1l1-Egfp } \\
\hline \multicolumn{2}{|c|}{ Master mix: } \\
\hline $1 \mu \mathrm{l}$ & DNA \\
\hline $0.75 \mu \mathrm{l}$ & se-primer \#22022 (10 pmol//ul) \\
\hline $0.75 \mu \mathrm{l}$ & as-primer \#15526 (10 pmol//ul) \\
\hline $2 \mu \mathrm{l}$ & dNTP (2 mM) \\
\hline $4 \mu \mathrm{l}$ & GoTaq buffer (5x) \\
\hline $0.1 \mu \mathrm{l}$ & GoTaq \\
\hline $11.4 \mu \mathrm{l}$ & $\mathrm{ddH}_{2} \mathrm{O}$ \\
\hline \multicolumn{2}{|c|}{ PCR program: } \\
\hline $1.94^{\circ} \mathrm{C}$ & $5 \mathrm{~min}$ \\
\hline 2. $94^{\circ} \mathrm{C}$ & $15 \mathrm{~s}$ \\
\hline $3.51^{\circ} \mathrm{C}$ & $30 \mathrm{~s}$ \\
\hline $4.72^{\circ} \mathrm{C}$ & $40 \mathrm{~s}$ \\
\hline \multicolumn{2}{|c|}{$\rightarrow$ Step 2 to 4 for 40 cycles } \\
\hline $5.72^{\circ} \mathrm{C}$ & $5 \min$ \\
\hline 6. $4^{\circ} \mathrm{C}$ & pause \\
\hline
\end{tabular}

\begin{tabular}{|c|c|}
\hline \multicolumn{2}{|c|}{ CaMKIla-Cre } \\
\hline \multicolumn{2}{|c|}{ Master mix: } \\
\hline $1 \mu \mathrm{l}$ & DNA \\
\hline $0.5 \mu \mathrm{l}$ & se-primer \#06722 (10 pmol/ $\mu \mathrm{l})$ \\
\hline $0.5 \mu \mathrm{l}$ & as-primer \#06723 (10 pmol/ $\mu \mathrm{l})$ \\
\hline $2 \mu \mathrm{l}$ & dNTP (2 mM) \\
\hline $4 \mu \mathrm{l}$ & GoTaq buffer (5x) \\
\hline $0.1 \mu \mathrm{l}$ & GoTaq \\
\hline
\end{tabular}

\begin{tabular}{|r|l|}
\hline \multicolumn{2}{|l|}{ Fdft1-flox } \\
\hline \multicolumn{2}{|l|}{ Master mix: } \\
\hline $1 \mu \mathrm{l}$ & DNA \\
\hline $0.5 \mu \mathrm{l}$ & se-primer \#02884 (10 pmol/ $\mu \mathrm{l})$ \\
\hline $0.5 \mu \mathrm{l}$ & as-primer \#32822 (10 pmol/ $\mu \mathrm{l})$ \\
\hline $0.5 \mu \mathrm{l}$ & as-primer2 \#02720(10 pmol/ $\mu \mathrm{l})$ \\
\hline $2 \mu \mathrm{l}$ & dNTP $(2 \mathrm{mM})$ \\
\hline $4 \mu \mathrm{l}$ & GoTaq buffer (5x) \\
\hline
\end{tabular}




\begin{tabular}{|c|c|}
\hline $11.9 \mu \mathrm{l}$ & $\mathrm{ddH}_{2} \mathrm{O}$ \\
\hline \multicolumn{2}{|c|}{ PCR program: } \\
\hline $1.94^{\circ} \mathrm{C}$ & $2 \min$ \\
\hline $2.58^{\circ} \mathrm{C}$ & $30 \mathrm{~s}$ \\
\hline $3.72^{\circ} \mathrm{C}$ & $1 \mathrm{~min} 20 \mathrm{~s}$ \\
\hline $4.94^{\circ} \mathrm{C}$ & $30 \mathrm{~s}$ \\
\hline \multicolumn{2}{|c|}{$\rightarrow$ Step 2 to 4 for 30 cycles } \\
\hline $5.58^{\circ} \mathrm{C}$ & $1 \mathrm{~min}$ \\
\hline $6.72^{\circ} \mathrm{C}$ & $2 \min$ \\
\hline 7. $4^{\circ} \mathrm{C}$ & pause \\
\hline & \\
\hline
\end{tabular}

\begin{tabular}{|r|l|}
\hline $0.1 \mu \mathrm{l}$ & GoTaq \\
\hline $11.4 \mu \mathrm{l}$ & $\mathrm{ddH}_{2} \mathrm{O}$ \\
\hline PCR program: \\
\hline $1.95^{\circ} \mathrm{C}$ & $3 \mathrm{~min}$ \\
\hline $2.58^{\circ} \mathrm{C}$ & $30 \mathrm{~s}$ \\
\hline $3.72^{\circ} \mathrm{C}$ & $30 \mathrm{~s}$ \\
\hline $4.95^{\circ} \mathrm{C}$ & $45 \mathrm{~s}$ \\
\hline$\rightarrow^{\circ} \mathrm{Step} 2$ to 4 for 36 cycles \\
\hline $5.55^{\circ} \mathrm{C}$ & $1 \mathrm{~min}$ \\
\hline $6.72^{\circ} \mathrm{C}$ & 5 min \\
\hline $7.4^{\circ} \mathrm{C}$ & pause \\
\hline
\end{tabular}

\begin{tabular}{|c|c|}
\hline \multicolumn{2}{|l|}{ NG2-Eyfp } \\
\hline \multicolumn{2}{|c|}{ Master mix: } \\
\hline $1 \mu l$ & DNA \\
\hline $0.75 \mu \mathrm{l}$ & se-primer \#33262 (10 pmol/ $\mu \mathrm{l})$ \\
\hline $0.2 \mu l$ & as-primer \#03312 (10 pmol/ $\mu \mathrm{l})$ \\
\hline $0.6 \mu \mathrm{l}$ & $\begin{array}{l}\text { as-primer2 \#05039 } \\
(10 \mathrm{pmol} / \mu \mathrm{l})\end{array}$ \\
\hline $2 \mu l$ & dNTP (2 mM) \\
\hline $4 \mu \mathrm{l}$ & GoTaq buffer (5x) \\
\hline $0.1 \mu l$ & GoTaq \\
\hline $11.35 \mu \mathrm{l}$ & $\mathrm{dd} \mathrm{H}_{2} \mathrm{O}$ \\
\hline \multicolumn{2}{|c|}{ PCR program: } \\
\hline $1.95^{\circ} \mathrm{C}$ & $3 \mathrm{~min}$ \\
\hline $2.58^{\circ} \mathrm{C}$ & $30 \mathrm{~s}$ \\
\hline 3. $72^{\circ} \mathrm{C}$ & $1 \mathrm{~min}$ \\
\hline $4.95^{\circ} \mathrm{C}$ & $30 \mathrm{~s}$ \\
\hline \multicolumn{2}{|c|}{$\rightarrow$ Step 2 to 4 for 39 cycles } \\
\hline $5.58^{\circ} \mathrm{C}$ & $40 \mathrm{~s}$ \\
\hline $6.72^{\circ} \mathrm{C}$ & $5 \mathrm{~min}$ \\
\hline 7. $4^{\circ} \mathrm{C}$ & pause \\
\hline
\end{tabular}

\begin{tabular}{|c|c|}
\hline \multicolumn{2}{|c|}{ Slc1a3-CreERT2 } \\
\hline \multicolumn{2}{|c|}{ Master mix: } \\
\hline $1 \mu \mathrm{l}$ & DNA \\
\hline $1 \mu \mathrm{l}$ & se-primer \#26605 (10 pmol/ $\mu \mathrm{l})$ \\
\hline $1 \mu \mathrm{l}$ & as-primer \#26606 (10 pmol/ $\mu \mathrm{l})$ \\
\hline $0.5 \mu \mathrm{l}$ & as-primer2 \#26607 (10 pmol/ $/ \mu \mathrm{l})$ \\
\hline $2 \mu \mathrm{l}$ & dNTP (2 mM) \\
\hline $4 \mu \mathrm{l}$ & GoTaq buffer (5x) \\
\hline $0.1 \mu \mathrm{l}$ & GoTaq \\
\hline $10.4 \mu \mathrm{l}$ & $\mathrm{ddH}_{2} \mathrm{O}$ \\
\hline \multicolumn{2}{|c|}{ PCR program: } \\
\hline $1.95^{\circ} \mathrm{C}$ & $3 \min$ \\
\hline 2. $58^{\circ} \mathrm{C}$ & $30 \mathrm{~s}$ \\
\hline 3. $72^{\circ} \mathrm{C}$ & $45 \mathrm{~s}$ \\
\hline $4.95^{\circ} \mathrm{C}$ & $30 \mathrm{~s}$ \\
\hline \multicolumn{2}{|c|}{$\rightarrow$ Step 2 to 4 for 35 cycles } \\
\hline $5.58^{\circ} \mathrm{C}$ & $1 \mathrm{~min}$ \\
\hline 6. $72^{\circ} \mathrm{C}$ & $10 \mathrm{~min}$ \\
\hline 7. $4^{\circ} \mathrm{C}$ & pause \\
\hline
\end{tabular}




\begin{tabular}{|c|c|}
\hline \multicolumn{2}{|c|}{ ROSA26-Tdtomato } \\
\hline \multicolumn{2}{|c|}{ Master mix: } \\
\hline $1 \mu \mathrm{l}$ & DNA \\
\hline $0.5 \mu \mathrm{l}$ & se-primer \#14025 (10 pmol/ $/ \mathrm{l})$ \\
\hline $0.5 \mu l$ & as-primer \#14024 (10 pmol/ $\mu \mathrm{l})$ \\
\hline $0.5 \mu l$ & $\begin{array}{l}\text { as-primer2 \#14026 } \\
(10 \mathrm{pmol} / \mu \mathrm{l})\end{array}$ \\
\hline $2 \mu \mathrm{l}$ & dNTP (2 mM) \\
\hline $4 \mu \mathrm{l}$ & GoTaq buffer (5x) \\
\hline $0.1 \mu \mathrm{l}$ & GoTaq \\
\hline $11.4 \mu \mathrm{l}$ & $\mathrm{dd} \mathrm{H}_{2} \mathrm{O}$ \\
\hline \multicolumn{2}{|c|}{ PCR program: } \\
\hline $1.95^{\circ} \mathrm{C}$ & $3 \min$ \\
\hline $2.58^{\circ} \mathrm{C}$ & $30 \mathrm{~s}$ \\
\hline $3.72^{\circ} \mathrm{C}$ & $1 \mathrm{~min}$ \\
\hline $4.95^{\circ} \mathrm{C}$ & $30 \mathrm{~s}$ \\
\hline \multicolumn{2}{|c|}{$\rightarrow$ Step 2 to 4 for 39 cycles } \\
\hline $5.58^{\circ} \mathrm{C}$ & $40 \mathrm{~s}$ \\
\hline 6. $72^{\circ} \mathrm{C}$ & $5 \mathrm{~min}$ \\
\hline 7. $4^{\circ} \mathrm{C}$ & pause \\
\hline
\end{tabular}

\begin{tabular}{|c|c|}
\hline \multicolumn{2}{|c|}{ ROSA26-Eyfp } \\
\hline \multicolumn{2}{|c|}{ Master mix: } \\
\hline $1 \mu \mathrm{l}$ & DNA \\
\hline $0.5 \mu \mathrm{l}$ & se-primer \#03735 (10 pmol/ $\mu \mathrm{l})$ \\
\hline $0.5 \mu \mathrm{l}$ & as-primer \#03736 (10 pmol/ $\mu \mathrm{l})$ \\
\hline $0.5 \mu \mathrm{l}$ & as-primer2 \#03737 (10 pmol/ $\mu \mathrm{l})$ \\
\hline $2 \mu \mathrm{l}$ & dNTP (2 mM) \\
\hline $4 \mu \mathrm{l}$ & GoTaq buffer (5x) \\
\hline $0.1 \mu \mathrm{l}$ & GoTaq \\
\hline $11.4 \mu \mathrm{l}$ & $\mathrm{ddH}_{2} \mathrm{O}$ \\
\hline \multicolumn{2}{|c|}{ PCR program: } \\
\hline 1. $95^{\circ} \mathrm{C}$ & $3 \min$ \\
\hline 2. $56^{\circ} \mathrm{C}$ & $30 \mathrm{~s}$ \\
\hline 3. $72^{\circ} \mathrm{C}$ & $45 \mathrm{~s}$ \\
\hline $4.95^{\circ} \mathrm{C}$ & $30 \mathrm{~s}$ \\
\hline \multicolumn{2}{|c|}{$\rightarrow$ Step 2 to 4 for 39 cycles } \\
\hline $5.56^{\circ} \mathrm{C}$ & $1 \mathrm{~min}$ \\
\hline 6. $72^{\circ} \mathrm{C}$ & $10 \mathrm{~min}$ \\
\hline 7. $4^{\circ} \mathrm{C}$ & pause \\
\hline
\end{tabular}

\begin{tabular}{|r|l|}
\hline \multicolumn{2}{|l|}{ 5xFAD } \\
\hline \multicolumn{2}{|l|}{ Master mix: } \\
\hline $1 \mu \mathrm{l}$ & DNA \\
\hline $0.5 \mu \mathrm{l}$ & se-primer \#19746 (10 pmol/ $\mu \mathrm{l})$ \\
\hline $0.5 \mu \mathrm{l}$ & as-primer \#19747 (10 pmol/ $\mu \mathrm{l})$ \\
\hline $2 \mu \mathrm{l}$ & dNTP $(2 \mathrm{mM})$ \\
\hline $4 \mu \mathrm{l}$ & GoTaq buffer $(5 \mathrm{x})$ \\
\hline $0.1 \mu \mathrm{l}$ & GoTaq \\
\hline $11.9 \mu \mathrm{l}$ & dd $\mathrm{H}_{2} \mathrm{O}$ \\
\hline PCR program: \\
\hline
\end{tabular}




\begin{tabular}{|r|l|}
\hline $1.95^{\circ} \mathrm{C}$ & $3 \mathrm{~min}$ \\
\hline $2.66^{\circ} \mathrm{C}$ & $25 \mathrm{~s}$ \\
\hline $3.72^{\circ} \mathrm{C}$ & $40 \mathrm{~s}$ \\
\hline $4.95^{\circ} \mathrm{C}$ & $25 \mathrm{~s}$ \\
\hline$\rightarrow$ & $\mathrm{Step} 2$ to 4 for 36 cycles \\
\hline $5.66^{\circ} \mathrm{C}$ & 1 min \\
\hline $6.72^{\circ} \mathrm{C}$ & 10 min \\
\hline $7.4^{\circ} \mathrm{C}$ & pause \\
\hline
\end{tabular}

\subsection{Tamoxifen administration}

Tamoxifen (Sigma, T5648) was dissolved in corn oil (Sigma, C8267) at a concentration of $7.5 \mathrm{mg} / \mathrm{ml}$ and injected intraperitoneally at $75 \mu \mathrm{g} / \mathrm{g}$ body weight on 5 consecutive days. In order to assess recombination in adult animals tamoxifen was administered to male and female mice at the age of 7-10 weeks unless stated otherwise. These mice were analyzed 12 (immunohistochemistry) and 20 days (electrophysiology) after the first tamoxifen injection. In astroSQS mutants, astroSQS-5XFAD mutants and corresponding controls tamoxifen was administered at P35 and animals were analyzed at P64 unless stated otherwise.

\subsection{Protein analyses}

\subsubsection{Sample preparation}

Animals were sacrificed by cervical dislocation, peripheral organs (lung, liver, kidney, small intestine, heart) were extracted and snap frozen on dry ice. The thoracic spinal cord was extracted from the surrounding vertebrae and snap frozen on dry ice. The brain was quickly but carefully removed from the skull, the meninges were removed and the brain was immediately cooled down in 1x DPBS (Gibco, 14190-094) on ice. Using a cooled brain matrix the brain was sliced in approx. $1 \mathrm{~mm}$ sagittal sections. While keeping the sections cooled and covered with 1x PBS, 
regions of interest (cortex, hippocampus, cerebellum) were prepared and snap frozen on dry ice. Tissue samples were stored at $-80^{\circ} \mathrm{C}$ until further processing.

\subsubsection{Tissue lysate preparation using sucrose buffer}

For protein and mRNA analyses tissue samples were homogenized in pre-cooled sucrose buffer containing protease and phosphatase inhibitors (cOmplete ${ }^{\mathrm{TM}}$, PhosSTOPTM, Roche) using the PT1200E homogenizer (Polytron) at max. speed for 20 seconds. For cortex samples $1000 \mu \mathrm{l}$, for hippocampus samples $300 \mu \mathrm{l}$ and for peripheral organs 300-1000 $\mu \mathrm{l}$ of sucrose buffer were used for homogenization. For RNA purification $100 \mu$ lysate were immediately transferred to $600 \mu \mathrm{l}$ RLT buffer (Qiagen, 79216), vortexed and stored at $-20^{\circ} \mathrm{C}$ until use. For Western blot analyses lysates were aliquoted and snap frozen on dry ice. Samples were stored at $-80^{\circ} \mathrm{C}$ until use.

\subsubsection{Tissue lysate preparation using TBS and SDS buffers}

Hippocampal lysates of conditional 5xFAD mutants and controls were prepared by sequential protein extraction as described previously (Hüttenrauch et al., 2017) with slight modifications. Frozen tissue samples were weighed and homogenized in TBS buffer ( $6 \mu \mathrm{l} / \mathrm{mg}$ tissue) containing protease inhibitors (cOmplete ${ }^{\mathrm{TM}}$, Roche) using the PT1200E homogenizer (Polytron) at max. speed for 20 seconds. Lysates were then centrifuged at $17400 \times \mathrm{g}$ at $4^{\circ} \mathrm{C}$ for $20 \mathrm{~min}$ (Optima ${ }^{\mathrm{TM}}$ TLX 120 Ultracentrifuge, Beckman Coulter), the supernatant containing TBS-soluble proteins was completely removed, aliquoted and frozen on dry ice. Subsequently, $200 \mu$ of $2 \%$ SDS buffer (incl. protease inhibitors) were added to the pellet followed by sonication until complete resuspension of the pellet and centrifugation at $17400 \mathrm{xg}$ at $4^{\circ} \mathrm{C}$ for 20 min. The supernatant containing SDS-soluble proteins was aliquoted and frozen on dry ice. All samples were stored at $-80^{\circ} \mathrm{C}$ until use.

\subsubsection{Measurement of protein concentration}

To determine total protein concentration of tissue lysates the DC ${ }^{T M}$ Protein Assay kit (BIO-RAD, 5000112) was used which is based on the method described by Lowry 
and colleagues (Lowry et al., 1951). Samples were analyzed in triplicates in Falcon® 96-well flat bottom plates (Corning Incorporated, 353072) according to the manufacturer's Microplate Assay Protocol. Absorbance was measured after 15 min of incubation at $736 \mathrm{~nm}$ using the Eon plate reader (Biotek). Protein concentrations were calculated by means of standard curves generated by serial dilutions of bovine serum albumin (BSA, BioLabs, B9000S) diluted in the respective buffers.

\subsubsection{Protein separation using SDS-PAGE}

Discontinuous sodium dodecyl sulfate polyacrylamide gel electrophoresis (SDSPAGE) initially described by Laemmli (Laemmli, 1970) was used to separate the proteins of a sample by size. SDS polyacrylamide gels of $1.5 \mathrm{~mm}$ thickness were casted using the Mini-PROTEAN ${ }^{\mathrm{TM}}$ Handcast System (BIO-RAD). The separation gel solution (10\% or $12 \%$ acrylamide) was overlayed with isopropyl alcohol and allowed to polymerize for $30 \mathrm{~min}$. After rinsing with $\mathrm{ddH}_{2} \mathrm{O}$ the stacking gel solution was poured on the separation gel, a comb (10- or 15-well) was inserted and the gel was left to polymerize for $30 \mathrm{~min}$. Tissue lysates homogenized in sucrose buffer were adjusted to equal protein concentration and mixed with 4x SDS buffer and dithiothreitol (DTT, final concentration $25 \mathrm{mM}$ ) and denatured at $70^{\circ} \mathrm{C}$ for $10 \mathrm{~min}$. Samples were loaded at $10-25 \mu \mathrm{g} /$ well as well as $5 \mu \mathrm{l}$ of pre-stained protein ladder (PageRuler ${ }^{\mathrm{TM}}$, ThermoFisher Scientific, 26619) and gels were run in $1 \mathrm{x}$ running buffer using the Mini-PROTEAN 3 chamber (BIO-RAD) connected to a power supply (BIO-RAD) at constant $200 \mathrm{~V}$ for $45-60$ min depending on the size of the proteins of interest.

\subsubsection{Separation of APP C-terminal fragments}

In order to separate the small APP C-terminal fragments $(\sim 12-14 \mathrm{kDa})$ a different gel-buffer system was used. SDS-soluble protein fractions of hippocampal lysates were mixed with $4 x$ reducing sample buffer (Schägger, 2006) using DTT instead of $\beta$-mercaptoethanol and adjusted to a final protein concentration of $2 \mu \mathrm{g} / \mu \mathrm{l}$ with $\mathrm{ddH}_{2} \mathrm{O}$. Samples were then denatured at $70^{\circ} \mathrm{C}$ for $10 \mathrm{~min}$ and $20 \mu \mathrm{g} / \mathrm{well}$ sample (unless stated otherwise) as well as $5 \mu$ l of pre-stained protein ladder (PageRuler ${ }^{\mathrm{TM}}$, ThermoFisher Scientific, 26619) were loaded onto NuPAGETM 12\% Bis-Tris Protein 
Gels (ThermoFisher Scientific, NP0341BOX). Gels were run in 1x MES buffer using the XCell SureLock ${ }^{\mathrm{TM}}$ Mini-Cell Electrophoresis System (ThermoFisher Scientific) connected to a power supply (BIO-RAD) at constant $200 \mathrm{~V}$ for $60 \mathrm{~min}$.

\subsection{Western blotting}

\subsubsection{Electrophoretic transfer}

Proteins were transferred to PVDF membranes (GE Healthcare, 10600023) using a tank blot system (XCell SureLock ${ }^{\mathrm{TM}}$ Mini-Cell Electrophoresis System, ThermoFisher Scientific). PVDF membranes were incubated for 1 min in methanol followed by rinsing two times with $\mathrm{dd}_{2} \mathrm{O}$. In order to provide sufficient buffer, blotting papers (GE Healthcare, 3030917) and blotting pads (ThermoFisher Scientific, El9052) were pre-soaked in $1 \mathrm{x}$ transfer buffer. The blotting sandwich was placed on the cathode core of the blotting system (4x blotting paper $\rightarrow$ gel $\rightarrow$ membrane $\rightarrow 4 \mathrm{x}$ blotting paper $\rightarrow 6 \mathrm{x}$ blotting pad), the chamber was filled with $1 \mathrm{x}$ transfer buffer and blotting was performed at $38 \mathrm{~V}$ for $60 \mathrm{~min}$ at $\mathrm{RT}$

\subsubsection{Immunodetection of proteins on PVDF membranes}

After blotting PVDF membranes were incubated in $1 \times$ TBS containing $0.05 \%$ Tween20 (Promega, H5151, 1x TBS-T) and 5\% w/v skim milk powder (blocking buffer) for 60 min under constant shaking at RT. Subsequently, membranes were transferred to $50 \mathrm{ml}$ Falcon tubes containing $5 \mathrm{ml}$ of primary antibodies diluted in blocking buffer and incubated under constant rotation overnight at $4^{\circ} \mathrm{C}$. On the next day membranes were washed 3 times with $1 \times$ TBS-T for 10 min on a shaker at RT and then incubated in $50 \mathrm{ml}$ Falcon tubes containing $5 \mathrm{ml}$ of horseradish peroxidase (HRP)-coupled secondary antibodies diluted in blocking buffer for $1 \mathrm{~h}$ under constant rotation at RT. After washing 3 times with $1 \times$ TBS-T for 10 min membranes are incubated for 1 min in Enhanced Chemiluminescence solution (Western Lightning ${ }^{\circledR}$ Plus, Perkin Elmer) followed by signal detection using the ChemoCam Imager HR 16-3200 (Intas). 


\subsubsection{Densitometric quantification of immunoreactive bands}

Densitometric analysis of Western blot bands was performed on pictures taken with the ChemoCam Imager HR 16-3200 system using the gel analyzing function of the NIH ImageJ 1.50e software or using Image Studio 5.2 Lite (Li-Cor Biosciences). Results were normalized to loading controls (Actin or GAPDH) by calculating relative density values of the control protein and then dividing each density value of the protein band of interest by the corresponding relative density value of the control protein. Data were expressed as fold change to control animals and analyzed for statistical significance using GraphPad Prism 7 software.

\subsection{Electrochemiluminescence-linked immunoassay for $A \beta$}

For quantification of $A \beta 40$ and $A \beta 42$ peptides TBS- and SDS-soluble protein fractions of hippocampal lysates (see 5.4.3) were adjusted to equal protein concentrations $(1.6 \mu \mathrm{g} / \mu \mathrm{l}$ for TBS samples and $3.6 \mu \mathrm{g} / \mu \mathrm{l}$ for SDS samples) using the respective buffers. Samples were then diluted in Assay Diluent 35 (TBS samples 1:10, SDS samples 1:100) and analyzed in duplicates using the V-PLEX A $\beta$ Peptide Panel 1 (6E10) Kit and the MESO QuickPlex SQ 120 instrument (Mesoscale Discovery).

\subsection{Fluorescence-activated cell sorting (FACS)}

Animals were sacrificed by cervical dislocation, meninges were removed, and cortices were prepared into small pieces on ice and subsequently transferred to precooled tissue storage solution. Tissue samples were centrifuged at $900 \mathrm{rpm}$ (Heraeus Labofuge 400) for $1 \mathrm{~min}$ at $4^{\circ} \mathrm{C}$, the supernatant was removed and the samples were incubated in pre-warmed, carbogenated digest solution in a water bath at $37^{\circ} \mathrm{C}$ under constant carbogen supply for $30 \mathrm{~min}$. Samples were subsequently centrifuged at $900 \mathrm{rpm}$ for $1 \mathrm{~min}$ at $4^{\circ} \mathrm{C}$ and the supernatant was discarded. Papain digest was stopped by adding $15 \mathrm{ml}$ inhibitor solution to the samples followed by centrifugation (900 rpm, 10min) and removal of the supernatant. Tissue pieces were homogenized three times by trituration in fresh tissue storage solution, followed by washing with inhibitor solution and centrifugation 
at $900 \mathrm{rpm}$ for $10 \mathrm{~min}$ at $4^{\circ} \mathrm{C}$. To separate astrocytes from myelin and other cells, tissue homogenates were underlayed sequentially with 20,40 and $60 \%$ isotonic Percoll solution and centrifuged at $2300 \mathrm{rpm}$ for $40 \mathrm{~min}$ at $4^{\circ} \mathrm{C}$. The interface containing astrocytes was removed, mixed with $10 \mathrm{ml}$ FACS buffer and centrifuged for $10 \mathrm{~min}$ at $4^{\circ} \mathrm{C}$. Cells were resuspended in $200 \mu \mathrm{FACS}$ buffer and stained with APC-labeled ACSA2 antibody (Miltenyi Biotec, 130-102-315) to identify astrocytes. Flow cytometry was performed using a FACS Aria flow cytometer (BD Biosciences).

\subsection{Magnetic cell isolation (MACS)}

Magnetic cell isolation of cortical astrocytes of astroSQS and control animals was performed as previously described (Berghoff et al., 2017a). Briefly, dissected cortices were processed according to the adult brain dissociation protocol (Miltenyi Biotec), followed by antibody labeling and magnetic isolation of astrocytes according to the Microbead kit protocol (Miltenyi Biotec, ACSA-2, 130-097-679). Isolated astrocytes were transferred to RLT buffer for RNA isolation (see below).

\subsection{RNA isolation, cDNA synthesis and quantitative RT-PCR}

\subsubsection{RNA isolation from tissue samples}

RNA was isolated from tissue lysates that had previously been transferred to RLT buffer directly after homogenization using the RNeasy Mini Kit (Qiagen, 74106) according to the manufacturer's instructions with slight modifications. Equal amounts of $70 \%$ ethanol were added to the sample, vortexed for 15 seconds and transferred to RNeasy columns. After centrifugation for $1 \mathrm{~min}$ at $13000 \mathrm{rpm}$ (Heraeus Biofuge Pico) the flow through was discarded and $350 \mu$ l buffer RW1 were added to the columns. Samples were centrifuged at $13000 \mathrm{rpm}$ for $1 \mathrm{~min}$, the flow through discarded and DNAse digestion was performed for $15 \mathrm{~min}$ at RT. Subsequently, columns were again washed with $350 \mu \mathrm{l}$ buffer RW 1 and flow through as well as collection tubes were discarded after centrifugation at $13000 \mathrm{rpm}$ for $1 \mathrm{~min}$. Columns were placed in new collection tubes and washed two times with 500 $\mu \mathrm{l}$ of buffer RPE by centrifugation at $13000 \mathrm{rpm}$ for $1 \mathrm{~min}$ after which flow through and collection tubes were discarded. Columns were placed in new collection tubes 
and dried by centrifugation at $13000 \mathrm{rpm}$ for 2 min. Finally, RNA was eluted two times with the same $30 \mu \mathrm{l}$ of RNA grade $\mathrm{H}_{2} \mathrm{O}$ by centrifugation at $13000 \mathrm{rpm}$ for 1 min. Quality and concentration of RNA was measured using RNA Nano (Agilent) or a NanoDrop spectrophotometer.

\subsubsection{RNA isolation from MACS-purified samples}

MACS-purified cell lysates in RLT buffer were thawed rapidly in a water bath at $37^{\circ} \mathrm{C}$ followed by thorough vortexing. Cell lysates were processed using QIAshredder (Qiagen, 79654) and subsequently the RNeasy Micro Kit (Qiagen, 74004) including DNAse digest according to the manufacturer's protocols. RNA was eluted by adding $50 \mu$ of RNA grade $\mathrm{H}_{2} \mathrm{O}$ to the columns, followed by 5 min incubation at RT and centrifugation for $1 \mathrm{~min}$ at $13000 \mathrm{rpm}$ (Heraeus Biofuge Pico). A second volume of $50 \mu \mathrm{l}$ of fresh RNA grade $\mathrm{H}_{2} \mathrm{O}$ was added followed by centrifugation for $1 \mathrm{~min}$ at $13000 \mathrm{rpm}$ to elute residual RNA from the columns.

\subsubsection{RNA purification by precipitation}

RNA isolated from MACS-purified cells was purified by precipitation in order to maximize RNA concentration for cDNA synthesis. First, $1 \mu \mathrm{l}$ of glycogen $(20 \mu \mathrm{g} / \mu \mathrm{l}$, Roche, 10901393001) were added to $100 \mu$ of eluted RNA followed by vortexing. Subsequently, $50 \mu \mathrm{l} 7.5 \mathrm{M}$ ammonium acetate were added followed by vortexing and thorough mixing with $375 \mu \mathrm{l}$ of $100 \%$ ethanol. Samples were left on ice for 30 min and then centrifuged for $20 \mathrm{~min}$ at $13000 \mathrm{rpm}$ (Heraeus Biofuge Pico). The supernatant was carefully removed and pellets were washed with $1 \mathrm{ml}$ of $70 \%$ ethanol in RNA grade $\mathrm{H}_{2} \mathrm{O}$ by shaking and subsequent centrifugation for $5 \mathrm{~min}$ at $13000 \mathrm{rpm}$. Afterwards, the supernatant was carefully removed and pellets were dried for approx. 5 min at RT to remove residual ethanol. Finally, $4 \mu \mathrm{l}$ of RNA grade $\mathrm{H}_{2} \mathrm{O}$ were added followed by incubation for $5 \mathrm{~min}$ on ice and resuspension of the pellets. 


\subsubsection{Complementary single stranded DNA (cDNA) synthesis}

Isolated RNA from cells or tissue samples was transcribed to cDNA using reverse transcriptase (SuperScript ${ }^{\mathrm{TM}}$ III, ThermoFisher Scientific, 18080044) and a mixture of oligo-dT (0.06 $\mu \mathrm{M}$, in house no. \#09578) and random nonamer (12 $\mu \mathrm{M}$, in house no. \#04542) primers. RNA derived from tissue was used up to $1 \mu \mathrm{g}$ for cDNA synthesis whereas the entire sample was used in case of MACS-isolated cells. Synthesis was performed according to the manufacturer's instructions using the Thermocycler T3 (Biometra).

\subsubsection{Quantitative RT-PCR}

Quantitative RT-PCR was performed in triplicates on 384-well plates (Roche) using the GoTaq ${ }^{\circledR}$ qPCR Master Mix (Promega, A6002) and the LightCycler ${ }^{\circledR} 480$ Instrument (Roche). cDNA was diluted 1:5 in nuclease-free water (Promega) for MACS-isolated cells or used at $4 \mathrm{ng} /$ well according to the following mix:

\begin{tabular}{|r|l|}
\hline $2 \mu \mathrm{l}$ & cDNA \\
\hline $0.4 \mu \mathrm{l}$ & se primer $(10 \mathrm{pmol} / \mu \mathrm{l})$ \\
\hline $0.4 \mu \mathrm{l}$ & as primer $(10 \mathrm{pmol} / \mu \mathrm{l})$ \\
\hline $5 \mu \mathrm{l}$ & GoTaq® Mix \\
\hline $2.2 \mu \mathrm{l}$ & $\mathrm{H}_{2} \mathrm{O}$ \\
\hline
\end{tabular}

Background subtraction and thresholding was performed using the LightCycler® 480 software (Roche) and values were exported to MS Excel 2013. Expression of target genes was normalized to the housekeeping gene Rps13 (ribosomal protein S13) for MACS-isolated cells or to the mean of Rps13 and Rplp0 (60S acidic ribosomal protein $\mathrm{P}$ ) and analyzed using the $2^{-\Delta \Delta \mathrm{Ct}}$ method. 


\subsection{Histology}

\subsubsection{Perfusion and tissue fixation}

Mice were anesthetized by intraperitoneal injection of Avertin $(0.2 \mathrm{ml} / 10 \mathrm{~g}$ body weight) and $500 \mu \mathrm{l}$ of blood were collected by cardiac puncture directly followed by intracardial perfusion with PBS pre-warmed to $37^{\circ} \mathrm{C}$ for $10 \mathrm{~min}$. Mutant mice expressing the Tdtomato reporter were subsequently perfused with $4 \%$ paraformaldehyde (PFA) for 20 min. Afterwards, peripheral organs (heart, kidney, liver, lung, small intestine) as well as brain and spinal cord were removed followed by immersion fixation overnight at $4^{\circ} \mathrm{C}$. Tissues were stored in PBS containing $0.1 \%$ $\mathrm{v} / \mathrm{v}$ PFA and $0.1 \% \mathrm{w} / \mathrm{v}$ sodium azide in the dark at $4^{\circ} \mathrm{C}$ until sectioning. For all other mice the brain was removed after perfusion with PBS, hemispheres were dissected, immersion fixed in $4 \%$ PFA for $48 \mathrm{~h}$ at $4^{\circ} \mathrm{C}$ followed by processing of one hemisphere for cryosectioning and the other for paraffin sectioning.

\subsubsection{Vibratome and cryostat sectioning}

Brains from mutant mice carrying the Tdtomato reporter were cut into $40 \mu \mathrm{m}$ sagittal sections using either a Leica VT1000S or a Leica VT1200S vibratome (Leica Microsystems). For cryostat sectioning peripheral organs, spinal cord and hemispheres were cryoprotected in $15 \%$ and subsequently in $30 \%$ sucrose in PBS, frozen in embedding medium (Tissue-Tek®, 4583) on dry ice and stored at $-80^{\circ} \mathrm{C}$. Peripheral organs and spinal cord were cut on a cryostat (Leica) into $20 \mu \mathrm{m}$ and 14 $\mu \mathrm{m}$ thick sections, respectively. Frozen hemispheres of conditional 5xFAD mutants and controls were entirely cut into $40 \mu \mathrm{m}$ serial sections collecting every $12^{\text {th }}$ section in the same well of a Falcon® 12-well plate (Corning Incorporated, 353043) filled with anti-freeze solution. Sections were stored in anti-freeze solution at $-20^{\circ} \mathrm{C}$.

\subsubsection{Paraffin embedding and microtome sectioning}

Hemispheres of conditional 5xFAD mutants and controls were embedded in paraffin using the Microm HMP 110 instrument running the following program: 


$\begin{array}{ll}50 \% \text { ethanol } & 1 \mathrm{~h} \\ 70 \% \text { ethanol } & 2 \times 2 \mathrm{~h} \\ 96 \% \text { ethanol } & 2 \times 2 \mathrm{~h} \\ \text { 100\% ethanol } & 2 \times 2 \mathrm{~h} \\ \text { Isopropyl alcohol } & 1 \mathrm{~h} \\ \text { Xylene } & 2 \times 2 \mathrm{~h} \\ \text { Paraffin } & 2 \times 2 \mathrm{~h}\end{array}$

Subsequently, hemispheres were casted in blocks using $60^{\circ} \mathrm{C}$ warm paraffin, cooled to dry and stored at RT. Hemispheres were cut into $5 \mu \mathrm{m}$ thick sagittal sections using the HM 400 microtome (MICROM). Sections were dried overnight at $36^{\circ} \mathrm{C}$.

\subsubsection{Immunohistochemistry}

\subsubsection{Immunostaining of vibratome sections}

Free-floating sections were processed for immunohistochemistry by permeabilization in $0.4 \%$ Triton X-100 (Sigma, T8787-250ML) in PBS for $30 \mathrm{~min}$ followed by blocking in $4 \%$ horse serum (HS) and $0.2 \%$ Triton X-100 in PBS for 30 min. Primary antibody incubation was carried out at $4^{\circ} \mathrm{C}$ overnight or for $48 \mathrm{~h}$ (CAll and Olig2) in $1 \% \mathrm{HS}$ and $0.05 \%$ Triton X-100 in PBS. Incubation with secondary antibodies and DAPI (4',6-diamidino-2-phenylindole) was carried out at room temperature for $2 \mathrm{~h}$ in $1.5 \% \mathrm{HS}$ in PBS. Sections were mounted in AquaPolymount (Polysciences).

\subsubsection{Immunostaining of frozen sections}

\subsection{Staining of mounted sections}

Cryostat sections were collected on Superfrost $\AA^{\circledR}$ Plus slides (Thermo Scientific) and incubated in PBS for 5 min followed by blocking in 10\% HS and 0.5\% Triton X-100 in PBS for $30 \mathrm{~min}$. Sections were incubated with primary antibodies diluted in 10\% HS and $0.3 \%$ Triton $\mathrm{X}-100$ in PBS overnight at $4^{\circ} \mathrm{C}$. Incubation with secondary 
antibodies and DAPI was carried out at room temperature for $2 \mathrm{~h}$ in $10 \% \mathrm{HS}$ and 0.5\% Triton X-100 in PBS and sections were mounted in AquaPolymount.

\subsection{A $\beta$ staining of free-floating sections}

Sagittal sections $(40 \mu \mathrm{m})$ were transferred to Costar® Netwells ${ }^{\mathrm{TM}}$ (Corning Incorporated, 3478) and washed three times for 15 min with PBS to remove antifreeze solution. Sections were then incubated for 30 min in $0.3 \% \mathrm{H}_{2} \mathrm{O}_{2}$ in $1 \times \mathrm{PBS}$, followed by washing three times in $1 \mathrm{x}$ PBS for 5 min each. Antigen retrieval for $A \beta$ was performed by incubation in $88 \%$ formic acid for $10 \mathrm{~min}$, directly followed by washing three times in $1 \times$ PBS for 5 min each. Sections were permeabilized in $0.1 \%$ Triton X-100 three times for 10 min followed by blocking in $4 \%$ goat serum in $1 \mathrm{x}$ PBS for $1 \mathrm{~h}$. Subsequently, sections were incubated with the pan $A \beta$ antibody 211218 (Synaptic Systems) diluted 1:500 in 1\% goat serum overnight. The next day, sections were washed three times in $0.1 \%$ Triton X-100 in 1x PBS for 10 min each, followed by two washing steps in 1x PBS. Immunodetection was performed using the LSAB2 kit (Dako), followed by incubation with the HRP substrate 3,3'Diaminobenzidine (DAB) using the DAB Substrate Kit (Zytomed Systems GmbH, DAB530) for 30s. After washing in 1x PBS three times for $10 \mathrm{~min}$, sections were mounted on Superfrost ${ }^{\circledR}$ Plus slides (Thermo Scientific) and dried overnight at $37^{\circ} \mathrm{C}$. The next day, sections were counterstained with haematoxylin and dehydrated by decreasing ethanol dilutions (50\%, 70\%, 90\%, and $100 \%$ for 5 min each) followed by xylene/isopropyl alcohol (1:1, $5 \mathrm{~min}$ ) and two times xylene (10 min) before mounting (Eukitt).

\subsubsection{Immunostaining of paraffin sections}

Paraffin sections were incubated at $60^{\circ} \mathrm{C}$ for $10 \mathrm{~min}$ and deparaffinized two times in xylene and in xylene/isopropyl alcohol (1:1) for 10 min each. Sections were then rehydrated in increasing ethanol dilutions (100\%, 90\%, 70\%, 50\%) for 5 min each and finally incubated in $\mathrm{ddH}_{2} \mathrm{O}$ for 5 min. Antigen retrieval was performed by boiling sections in sodium citrate buffer $(0.01 \mathrm{M}, \mathrm{pH} 6.0)$ for $10 \mathrm{~min}$ in a microwave and for immunofluorescence additionally by incubation in 0.1\% Triton X-100 in PBS for 15 min. For $A \beta$ staining sections were additionally incubated in $88 \%$ formic acid for 3 
min at RT. For chromogenic immunostaining sections were then incubated in trisbuffered saline (Tris buffer) containing $2 \% \mathrm{w} / \mathrm{v}$ skim milk powder for $5 \mathrm{~min}$ and transferred to Shandon Coverplates (ThermoFisher Scientific) followed by washing with Tris buffer containing $2 \%$ milk. For immunofluorescence sections were incubated in $10 \% \mathrm{v} / \mathrm{v}$ FCS (fetal calf serum) and $4 \% \mathrm{w} / \mathrm{v}$ skim milk powder in PBS for $1 \mathrm{~h}$ at RT followed by incubation with primary antibodies in 10\% FCS in PBS overnight in a humid chamber at $4^{\circ} \mathrm{C}$. Chromogenic immunostaining was continued by applying 3\% hydrogen peroxide for 5 min followed by washing with Tris buffer containing 2\% milk and incubation in Protein Bock Serum-Free (Dako) for 10 min. Sections were then incubated with primary antibodies in Antibody Diluent (Dako) at $4^{\circ} \mathrm{C}$ overnight. Immunofluorescence was completed on the next by washing three times in $0.1 \%$ Triton X-100 in PBS for $5 \mathrm{~min}$ and for another $5 \mathrm{~min}$ in PBS followed by incubation with fluorescently labelled secondary antibodies and DAPI in 10\% FCS in PBS for $1 \mathrm{~h}$ at RT. Finally, sections were washed three times with PBS for 5 min and mounted using AquaPolymount. For chromogenic immunostaining sections were washed in Tris buffer containing $2 \%$ milk followed by immunodetection using the LSAB2 kit (Dako). First, sections were incubated with biotinylated secondary antibodies for $10 \mathrm{~min}$, washed in Tris buffer containing $2 \%$ milk and then incubated with streptavidin-coupled HRP for 10 min. Sections were then washed in Tris buffer without milk for $5 \mathrm{~min}$ followed by incubation with the HRP substrate 3,3'Diaminobenzidine (DAB) using the DAB Substrate Kit (Zytomed Systems GmbH, DAB530) for 5 to $15 \mathrm{~min}$ depending on staining intensity. Subsequently, sections were washed two times in $\mathrm{ddH}_{2} \mathrm{O}$, nuclei were stained with haematoxylin and sections were dehydrated by decreasing ethanol dilutions $(50 \%, 70 \%, 90 \%$, and $100 \%$ for $5 \mathrm{~min}$ each) followed by xylene/isopropyl alcohol (1:1,5 min) and two times xylene (10 $\mathrm{min}$ ) before mounting (Eukitt).

\subsection{Measurement of serum cholesterol}

Blood collected during perfusion was allowed to clot for $1 \mathrm{~h}$ at RT and serum was prepared by centrifugation for $10 \mathrm{~min}$ at $5000 \mathrm{rpm}$ (Heraeus Biofuge Pico). Cholesterol measurements were performed using the architectll system (Abbott Diagnostics) by technicians of the Central Laboratory for Clinical Chemistry at the University Medical Center, Göttingen. 


\subsection{EdU proliferation assay}

To label proliferating cells in adult animals, EdU $(50 \mu \mathrm{g} / \mathrm{g})$ dissolved in saline was injected intraperitoneally on 16 consecutive days. Staining of $50 \mu \mathrm{m}$ sagittal vibratome sections was performed using the Click-iT Plus EdU Alexa Fluor 647 Imaging Kit (ThermoFisher Scientific, C10640) following the manufacturer's instructions. Sections were additionally labeled using an NG2 (AN2, J. Trotter) and S100beta antibody (Abcam) as well as DAPI.

\subsection{Electrophysiology}

Acute forebrain slices from 8 weeks old wild type and astroSQS-Tdto (each $n=3$ ) mice were prepared as described previously (Schnell et al., 2015). Briefly, after deep isoflurane narcosis, animals were decapitated, the forebrain was prepared and placed in ice-cooled, carbogen-saturated $\left(95 \% \mathrm{O}_{2}, 5 \% \mathrm{CO}_{2}\right)$ artificial cerebrospinal fluid (aCSF; in mM: $118 \mathrm{NaCl}, 3 \mathrm{KCl}, 1.5 \mathrm{CaCl}_{2}, 1 \mathrm{MgCl}_{2}, 1 \mathrm{NaH}_{2} \mathrm{PO}_{4}, 25 \mathrm{NaHCO}_{3}$, and $30 \mathrm{D}$-glucose; $330 \mathrm{mosmol} / \mathrm{l}, \mathrm{pH} 7.4)$. Sagittal sections (300 $\mu \mathrm{m})$ were cut on a vibroslicer (VT1200 S, Leica) and stored in aCSF at $35-36^{\circ} \mathrm{C}$ for at least $30 \mathrm{~min}$. Sulforhodamine $101(\mathrm{SR} 101,1 \mu \mathrm{M})$ staining was performed for $20 \mathrm{~min}$ at $34^{\circ} \mathrm{C}$ followed by washing in aCSF for $10 \mathrm{~min}$ at $34^{\circ} \mathrm{C}$ as described previously (Kafitz et al., 2008; Schnell et al., 2012). Subsequently, slices were transferred to the recording chamber and kept submerged by a platinum grid with nylon fibers for mechanical stabilization. The chamber was mounted on an upright microscope (Axioscope FS, Zeiss Germany, 40x objective) and continuously perfused with aCSF at room temperature at a flow rate of $5-10 \mathrm{ml} / \mathrm{min}$. Astrocytes were identified by SR101 staining in epifluorescence illumination (white-LED, Lumencor Sola SE II). For documentation, images of recorded tdTomato-expressing cells were taken with a CCD camera (Sensicam, PCO) and Imaging workbench 6.0 software (Indec Biosystems). Whole-cell voltage-clamp recordings were obtained with a MultiClamp 700B Amplifier (Molecular Devices). Patch electrodes were pulled from borosilicate glass capillaries (Biomedical Instruments, Zülpich, Germany) using a horizontal pipette-puller (Zeitz-Instrumente, Germany). Electrodes were filled with (in mM) 125 $\mathrm{KCl}, 1 \mathrm{CaCl}_{2}, 2 \mathrm{MgCl}_{2}, 4 \mathrm{Na}_{2} \mathrm{ATP}, 10$ EGTA, 10 HEPES (pH adjusted to 7.2 with $\mathrm{KOH}$ ) leading to tip resistance of $2-6 \mathrm{M} \Omega$. Currents were low-pass filtered at $3 \mathrm{kHz}$, 
and sampled at $10 \mathrm{kHz}$ and recorded with pClamp 10 software (Molecular Devices) and stored for off-line analysis. Membrane potentials $\left(\mathrm{V}_{\mathrm{m}}\right)$ were recorded at steady state and astrocytes were voltage-clamped to $-80 \mathrm{mV}$ and characterized by a voltage step protocol. Therefore, cells were hyperpolarized by -80 to $-10 \mathrm{mV}$ and depolarized by $+10 \mathrm{mV}$ to $+110 \mathrm{mV}$ voltage steps (10 mV increment). Mean I-V curves were calculated and inward and outward currents were compared at maximal hyperpolarization $\left(\Delta \mathrm{I}=\mathrm{I}_{-80}-\mathrm{I}_{-160}\right)$ and maximal depolarization $\left(\Delta \mathrm{I}=\mathrm{I}_{30}-\mathrm{I}_{-80}\right)$. The membrane resistance $\left(R_{i}\right)$ was calculated from the change of the holding current in response to the first hyperpolarizing voltage step to $-90 \mathrm{mV}$. Statistical analysis was performed using 1way ANOVA.

\subsection{Assessment of blood-brain barrier permeability}

Injection of Evans Blue and sodium fluorescein as well as quantification of tracer extravasation was performed as previously described (Berghoff et al., 2017a). Injection of bodipy-cholesterol (Avanti Polar Lipids) and the measurement of extravasated bodipy-cholesterol was performed as described (Berghoff et al., 2017b).

\subsection{Microscopy and quantification of immunoreactivity}

Specimens were analyzed by epifluorescence microscopy using a Plan-Apochromat 20x/0.8 objective (Zeiss Axio Oberser.Z1 with ApoTome.2) and the ZEN 2 software (Zeiss). Confocal laser scanning microscopy was performed using a Leica SP2 equipped with a HC PL APO lambda blue 20x/0.7 objective or with a Leica SP5 (HCX PL APO CS 20x/0.7, HCX PL APO lambda blue 40x/1.25, HCX PL APO CS 100x/1.44 objectives) using the Leica Confocal Software (Leica Microsystems). Immunolabeling visualized by DAB was analyzed using an Axio Imager.Z1 (Zeiss) equipped with an AxioCam MRc3, 0.63x Camera Adaptor and the ZEN 2012 blue edition software using a 20x objective (Plan-Apochromat 20x/0.8). Images were processed with $\mathrm{NIH}$ ImageJ and quantification of areas (GFAP, pan $A \beta, A \beta 42$ ) was performed by applying a semi-automated ImageJ software macro for thresholding and color deconvolution. Fluorescent images of AQP4 and Iba1 immunolabeling were thresholded manually using $\mathrm{NIH}$ ImageJ. Quantification of $A \beta$ 
immunoreactivity using the pan $A \beta$ antibody (211218, Synpatic Systems) was performed on every $12^{\text {th }}$ section (see 5.11.4.2.2) of serial sections throughout the hippocampus of one hemisphere. Quantification of $A \beta 42$ deposition in the subiculum was performed on paraffin sections of the lateral hippocampus. Analysis was performed on sections that corresponded to at least $60 \mu \mathrm{m}$ distance in the hippocampus to avoid overlapping quantification. Analysis of A 42 deposition was performed by a different investigator blind to the experimental groups.

\subsection{Statistical analysis}

Statistical analysis was performed by unpaired Student's t-test for comparison of two groups while one-way ANOVA with Tukey's multiple comparison test was used for comparison of more than two groups using the GraphPad Prism 7 software. $P$ values in the figure legends are shown with ${ }^{*} p<0.05,{ }^{* *} p<0.01,{ }^{* * *} p<0.001,{ }^{* * * *} p$ $<0.0001$, or with $\# p<0.05, \# \# p<0.01, \# \# \#<<0.001, \# \# \# p<0.0001$ for significant differences relative to wild type controls. Outliers were identified using the Grubb's test $(\alpha=0.05)$ or the ROUT test $(Q=2 \%)$ of the GraphPad Prism 7 software. 


\section{References}

Abbott NJ, Rönnbäck L, Hansson E. Astrocyte-endothelial interactions at the bloodbrain barrier. Nature Reviews Neuroscience 7: 41-53, 2005.

Abdul-Hay SO, Sahara T, McBride M, Kang D, Leissring MA. Identification of BACE2 as an avid B-amyloid-degrading protease. Molecular Neurodegeneration 7: 46, 2012.

Aguzzi A, O'Connor T. Protein aggregation diseases: pathogenicity and therapeutic perspectives. Nature Reviews Drug Discovery 9: 237-48, 2010.

Allué JA, Sarasa L, Izco M, Pérez-Grijalba V, Fandos N, Pascual-Lucas M, Ogueta $S$, Pesini P, Sarasa M. Outstanding Phenotypic Differences in the Profile of Amyloid$\beta$ between Tg2576 and APPswe/PS1dE9 Transgenic Mouse Models of Alzheimer's Disease. Journal of Alzheimer's Disease 53: 773-785, 2016.

Alves L, Correia ASA, Miguel R, Alegria P, Bugalho P. Alzheimer's disease: a clinical practice-oriented review. Frontiers in Neurology 3: 63, 2012.

Ando S, Tanaka Y, Toyoda Y, Kon K. Turnover of Myelin Lipids in Aging Brain. Neurochemical Research 28: 5-13, 2003.

Anthony TE, Heintz N. The folate metabolic enzyme ALDH1L1 is restricted to the midline of the early CNS, suggesting a role in human neural tube defects. The Journal of Comparative Neurology 500: 368-83, 2007.

Atagi Y, Liu C-C, Painter MM, Chen X-F, Verbeeck C, Zheng H, Li X, Rademakers R, Kang SS, Xu H, Younkin S, Das P, Fryer JD, Bu G. Apolipoprotein E Is a Ligand for Triggering Receptor Expressed on Myeloid Cells 2 (TREM2). Journal of Biological Chemistry 290: 26043-26050, 2015.

Bailey CC, DeVaux LB, Farzan M. The Triggering Receptor Expressed on Myeloid Cells 2 Binds Apolipoprotein E. Journal of Biological Chemistry 290: 26033-26042, 2015.

Bales KR, Verina T, Dodel RC, Du Y, Altstiel L, Bender M, Hyslop P, Johnstone EM, Little SP, Cummins DJ, Piccardo P, Ghetti B, Paul SM. Lack of apolipoprotein E dramatically reduces amyloid beta-peptide deposition. Nature Genetics 17: 263264, 1997.

Barrett PJ, Song Y, Van Horn WD, Hustedt EJ, Schafer JM, Hadziselimovic A, Beel AJ, Sanders CR. The amyloid precursor protein has a flexible transmembrane domain and binds cholesterol. Science 336: 1168-1171, 2012.

Basak JM, Verghese PB, Yoon H, Kim J, Holtzman DM. Low-density lipoprotein receptor represents an apolipoprotein $\mathrm{E}$-independent pathway of $A \beta$ uptake and degradation by astrocytes. Journal of Biological Chemistry 287: 13959-13971, 2012. 
Bateman RJ, Xiong C, Benzinger TLS, Fagan AM, Goate A, Fox NC, Marcus DS, Cairns NJ, Xie X, Blazey TM, Holtzman DM, Santacruz A, Buckles V, Oliver A, Moulder K, Aisen PS, Ghetti B, Klunk WE, McDade E, Martins RN, Masters CL, Mayeux R, Ringman JM, Rossor MN, Schofield PR, Sperling RA, Salloway S, Morris JC. Clinical and biomarker changes in dominantly inherited Alzheimer's disease. New England Journal of Medicine 367: 795-804, 2012.

Bell RD, Sagare AP, Friedman AE, Bedi GS, Holtzman DM, Deane R, Zlokovic BV. Transport pathways for clearance of human Alzheimer's amyloid beta-peptide and apolipoproteins $E$ and $J$ in the mouse central nervous system. Journal of Cerebral Blood Flow \& Metabolism 27: 909-918, 2006.

Bell RD, Winkler EA, Singh I, Sagare AP, Deane R, Wu Z, Holtzman DM, Betsholtz C, Armulik A, Sallstrom J, Berk BC, Zlokovic BV. Apolipoprotein E controls cerebrovascular integrity via cyclophilin A. Nature 485: 512-6, 2012.

Berghoff SA, Düking T, Spieth L, Winchenbach J, Stumpf SK, Gerndt N, Kusch K, Ruhwedel T, Möbius W, Saher G. Blood-brain barrier hyperpermeability precedes demyelination in the cuprizone model. Acta Neuropathologica Communications 5: 94, 2017a.

Berghoff SA, Gerndt N, Winchenbach J, Stumpf SK, Hosang L, Odoardi F, Ruhwedel T, Böhler C, Barrette B, Stassart R, Liebetanz D, Dibaj P, Möbius W, Edgar JM, Saher G. Dietary cholesterol promotes repair of demyelinated lesions in the adult brain. Nature Communications 8: 14241, $2017 \mathrm{~b}$.

Bertram L, McQueen MB, Mullin K, Blacker D, Tanzi RE. Systematic meta-analyses of Alzheimer disease genetic association studies: the AlzGene database. Nature Genetics 39: 17-23, 2007.

Björkhem I, Breuer O, Sakinis A, Wennmalm A. Importance of a novel oxidative mechanism for elimination of brain cholesterol. Turnover of cholesterol and 24(S)hydroxycholesterol in rat brain as measured with 1802 techniques in vivo and in vitro. Journal of Biological Chemistry 272: 30178-30184, 1997.

Björkhem I, Meaney S. Brain cholesterol: long secret life behind a barrier. Arteriosclerosis, thrombosis, and vascular biology 24: 806-15, 2004.

Brinkmann BG, Agarwal A, Sereda MW, Garratt AN, Müller T, Wende H, Stassart RM, Nawaz S, Humml C, Velanac V, Radyushkin K, Goebbels S, Fischer TM, Franklin RJ, Lai C, Ehrenreich H, Birchmeier C, Schwab MH. Neuregulin-1/ErbB signaling serves distinct functions in myelination of the peripheral and central nervous system. Neuron 59: 581-95, 2008.

Cahoy JD, Ben Emery, Kaushal A, Foo LC, Zamanian JL, Christopherson KS, Xing Y, Lubischer JL, Krieg PA, Krupenko SA, Thompson WJ, Ben Barres A. A transcriptome database for astrocytes, neurons, and oligodendrocytes: a new resource for understanding brain development and function. The Journal of Neuroscience 28: 264-78, 2008. 
Camargo N, Brouwers JF, Loos M, Gutmann DH, Smit AB, Verheijen MHG. Highfat diet ameliorates neurological deficits caused by defective astrocyte lipid metabolism. The FASEB Journal 26: 4302-15, 2012.

Cao D, Fukuchi K-I, Wan H, Kim H, Li L. Lack of LDL receptor aggravates learning deficits and amyloid deposits in Alzheimer transgenic mice. Neurobiology of Aging 27: 1632-1643, 2005.

Castellano JM, Deane R, Gottesdiener AJ, Verghese PB, Stewart FR, West T, Paoletti AC, Kasper TR, DeMattos RB, Zlokovic BV, Holtzman DM. Low-density lipoprotein receptor overexpression enhances the rate of brain-to-blood $A \beta$ clearance in a mouse model of $\beta$-amyloidosis. Proceedings of the National Academy of Sciences 109: 15502-15507, 2012.

Ceglia I, Reitz C, Gresack J, Ahn J-H, Bustos V, Bleck M, Zhang X, Martin G, Simon SM, Nairn AC, Greengard P, Kim Y. APP intracellular domain-WAVE1 pathway reduces amyloid- $\beta$ production. Nature Medicine 9: 1054-9, 2015.

Chow LML, Zhang J, Baker SJ. Inducible Cre recombinase activity in mouse mature astrocytes and adult neural precursor cells. Transgenic Research 17: 919-928, 2008.

Choy RW-Y, Cheng Z, Schekman R. Amyloid precursor protein (APP) traffics from the cell surface via endosomes for amyloid $\beta(A \beta)$ production in the trans-Golgi network. Proceedings of the National Academy of Sciences 109: E2077-82, 2012.

Christie KJ, Emery B, Denham M, Bujalka H, Cate HS, Turnley AM. Transcriptional regulation and specification of neural stem cells. Advances in Experimental Medicine and Biology 786: 129-155, 2013.

Cooper AD. Hepatic uptake of chylomicron remnants. Journal of Lipid Research 38: 2173-2192, 1997.

Cramer PE, Cirrito JR, Wesson DW, Lee CYD, Karlo JC, Zinn AE, Casali BT, Restivo JL, Goebel WD, James MJ, Brunden KR, Wilson DA, Landreth GE. ApoEdirected therapeutics rapidly clear $\beta$-amyloid and reverse deficits in $A D$ mouse models. Science 335: 1503-6, 2012.

Cummings JL, Zhong K, Kinney JW, Heaney C, Moll-Tudla J, Joshi A, Pontecorvo M, Devous M, Tang A, Bena J. Double-blind, placebo-controlled, proof-of-concept trial of bexarotene Xin moderate Alzheimer's disease. Alzheimer's Research \& Therapy 8: 4, 2016.

Cutler RG, Kelly J, Storie K, Pedersen WA, Tammara A, Hatanpaa K, Troncoso JC, Mattson MP. Involvement of oxidative stress-induced abnormalities in ceramide and cholesterol metabolism in brain aging and Alzheimer's disease. Proceedings of the National Academy of Sciences 101: 2070-2075, 2004.

Dallérac G, Chever O, Rouach N. How do astrocytes shape synaptic transmission? Insights from electrophysiology. Frontiers in Cellular Neuroscience 7: 159, 2013.

De Strooper B, Karran E. The Cellular Phase of Alzheimer's Disease. Cell 164: 603615, 2016. 
DeMattos RB, Brendza RP, Heuser JE, Kierson M, Cirrito JR, Fryer J, Sullivan PM, Fagan AM, Han X, Holtzman DM. Purification and characterization of astrocytesecreted apolipoprotein $E$ and $J$-containing lipoproteins from wild-type and human apoE transgenic mice. Neurochemistry International 39: 415-25, 2001.

Di Scala C, Chahinian H, Yahi N, Garmy N, Fantini J. Interaction of Alzheimer's $\beta$ amyloid peptides with cholesterol: mechanistic insights into amyloid pore formation. Biochemistry 53: 4489-4502, 2014.

Dietschy JM, Turley SD. Cholesterol metabolism in the brain. Current Opinion in Lipidology 2: 105-12, 2001.

Dietschy JM, Turley SD. Thematic review series: Brain Lipids. Cholesterol metabolism in the central nervous system during early development and in the mature animal. Journal of Lipid Research 45: 1375-1397, 2004.

Dominiczak MH, Caslake MJ. Apolipoproteins: metabolic role and clinical biochemistry applications. Annals of Clinical Biochemistry 48: 498-515, 2011.

Duyckaerts C, Delatour B, Potier M-C. Classification and basic pathology of Alzheimer disease. Acta Neuropathologica 118: 5-36, 2009.

Eberlé D, Hegarty B, Bossard P, Ferré P, Foufelle F. SREBP transcription factors: master regulators of lipid homeostasis. Biochimie 86: 839-848, 2004.

Eckman EA, Watson M, Marlow L, Sambamurti K, Eckman CB. Alzheimer's disease beta-amyloid peptide is increased in mice deficient in endothelin-converting enzyme. Journal of Biological Chemistry 278: 2081-2084, 2002.

Fagan AM, Holtzman DM, Munson G, Mathur T, Schneider D, Chang LK, Getz GS, Reardon CA, Lukens J, Shah JA, LaDu MJ. Unique lipoproteins secreted by primary astrocytes from wild type, apoE (-/-), and human apoE transgenic mice. Journal of Biological Chemistry 274: 30001-30007, 1999.

Ferrer I. Diversity of astroglial responses across human neurodegenerative disorders and brain aging. Brain Pathology 27: 645-674, 2017.

Ferris HA, Perry RJ, Moreira GV, Shulman GI, Horton JD, Kahn CR. Loss of astrocyte cholesterol synthesis disrupts neuronal function and alters whole-body metabolism. Proceedings of the National Academy of Sciences 114: 1189-1194, 2017.

Foo LC, Dougherty JD. Aldh1L1 is expressed by postnatal neural stem cells in vivo. Glia 61: 1533-41, 2013.

Fryer JD, Demattos RB, McCormick LM, O'Dell MA, Spinner ML, Bales KR, Paul SM, Sullivan PM, Parsadanian M, Bu G, Holtzman DM. The low density lipoprotein receptor regulates the level of central nervous system human and murine apolipoprotein $\mathrm{E}$ but does not modify amyloid plaque pathology in PDAPP mice. The Journal of Biological Chemistry 280: 25754-9, 2005.

Fukami S, Watanabe K, Iwata N, Haraoka J, Lu B, Gerard NP, Gerard C, Fraser P, Westaway D, George-Hyslop PS, Saido TC. Abeta-degrading endopeptidase, 
neprilysin, in mouse brain: synaptic and axonal localization inversely correlating with Abeta pathology. Neuroscience Research 43: 39-56, 2002.

Fünfschilling U, Jockusch WJ, Sivakumar N, Möbius W, Corthals K, Li S, Quintes S, Kim Y, Schaap IAT, Rhee J-S, Nave K-A, Saher G. Critical time window of neuronal cholesterol synthesis during neurite outgrowth. The Journal of Neuroscience 32: 7632-45, 2012.

Fünfschilling U, Saher G, Le Xiao, Möbius W, Nave K-A. Survival of adult neurons lacking cholesterol synthesis in vivo. BMC Neuroscience 8: 1, 2007.

Ganat YM, Silbereis J, Cave C, Ngu H, Anderson GM, Ohkubo Y, Ment LR, Vaccarino FM. Early postnatal astroglial cells produce multilineage precursors and neural stem cells in vivo. The Journal of Neuroscience 26: 8609-8621, 2006.

Ge W-P, Miyawaki A, Gage FH, Jan YN, Jan LY. Local generation of glia is a major astrocyte source in postnatal cortex. Nature 484: 376-80, 2012.

Goate A, Chartier-Harlin MC, Mullan M, Brown J, Crawford F, Fidani L, Giuffra L, Haynes A, Irving N, James L. Segregation of a missense mutation in the amyloid precursor protein gene with familial Alzheimer's disease. Nature 349: 704-706, 1991.

Halford RW, Russell DW. Reduction of cholesterol synthesis in the mouse brain does not affect amyloid formation in Alzheimer's disease, but does extend lifespan. Proceedings of the National Academy of Sciences of the United States of America 106: 3502-6, 2009.

Hardy J, Selkoe DJ. The amyloid hypothesis of Alzheimer's disease: progress and problems on the road to therapeutics. Science 297: 353-356, 2002.

Haynes SE, Hollopeter G, Yang G, Kurpius D, Dailey ME, Gan W-B, Julius D. The P2Y12 receptor regulates microglial activation by extracellular nucleotides. Nature Neuroscience 9: 1512-1519, 2006.

Heintz N. Gene expression nervous system atlas (GENSAT). Nature Neuroscience 7: 483, 2004.

Hickman SE, Kingery ND, Ohsumi TK, Borowsky ML, Wang L-C, Means TK, Khoury El J. The microglial sensome revealed by direct RNA sequencing. Nature Neuroscience 16: 1896-1905, 2013.

Hirrlinger PG, Scheller A, Braun C, Hirrlinger J, Kirchhoff F. Temporal control of gene recombination in astrocytes by transgenic expression of the tamoxifeninducible DNA recombinase variant CreERT2. Glia 54: 11-20, 2006.

Hirsch-Reinshagen V, Zhou S, Burgess BL, Bernier L, Mclsaac SA, Chan JY, Tansley GH, Cohn JS, Hayden MR, Wellington CL. Deficiency of ABCA1 impairs apolipoprotein E metabolism in brain. Journal of Biological Chemistry 279: 4119741207, 2004.

Holick MF. Vitamin D deficiency. New England Journal of Medicine 357: 266-281, 2007. 
Holtzman DM, Fagan AM, Mackey B, Tenkova T, Sartorius L, Paul SM, Bales K, Ashe $\mathrm{KH}$, Irizarry MC, Hyman BT. Apolipoprotein E facilitates neuritic and cerebrovascular plaque formation in an Alzheimer's disease model. Annals of Neurology 47: 739-747, 2000.

Holtzman DM, Herz J, Bu G. Apolipoprotein E and apolipoprotein E receptors: normal biology and roles in Alzheimer disease. Cold Spring Harbor Perspectives in Medicine 2: a006312, 2012.

Horton JD, Shimomura I, Brown MS, Hammer RE, Goldstein JL, Shimano H. Activation of cholesterol synthesis in preference to fatty acid synthesis in liver and adipose tissue of transgenic mice overproducing sterol regulatory element-binding protein-2. The Journal of Clinical Investigation 101: 2331-2339, 1998.

Hussain I, Powell D, Howlett DR, Tew DG, Meek TD, Chapman C, Gloger IS, Murphy KE, Southan CD, Ryan DM, Smith TS, Simmons DL, Walsh FS, Dingwall C, Christie G. Identification of a novel aspartic protease (Asp 2) as beta-secretase. Molecular and Cellular Neuroscience 14: 419-427, 1999.

Hussain I, Powell DJ, Howlett DR, Chapman GA, Gilmour L, Murdock PR, Tew DG, Meek TD, Chapman C, Schneider K, Ratcliffe SJ, Tattersall D, Testa TT, Southan C, Ryan DM, Simmons DL, Walsh FS, Dingwall C, Christie G. ASP1 (BACE2) cleaves the amyloid precursor protein at the beta-secretase site. Molecular and Cellular Neuroscience 16: 609-619, 2000.

Hüttenrauch M, Baches S, Gerth J, Bayer TA, Weggen S, Wirths O. Neprilysin Deficiency Alters the Neuropathological and Behavioral Phenotype in the 5XFAD Mouse Model of Alzheimer's Disease. Journal of Alzheimer's Disease 44, 2015.

Hüttenrauch $\mathrm{M}$, Walter S, Kaufmann M, Weggen S, Wirths O. Limited Effects of Prolonged Environmental Enrichment on the Pathology of 5XFAD Mice. Molecular Neurobiology 54: 6542-6555, 2017.

Jahn HM, Scheller A, Kirchhoff F. Genetic control of astrocyte function in neural circuits. Frontiers in Cellular Neuroscience 9: 310, 2015.

Jawhar S, Trawicka A, Jenneckens C, Bayer TA, Wirths O. Motor deficits, neuron loss, and reduced anxiety coinciding with axonal degeneration and intraneuronal $A \beta$ aggregation in the 5XFAD mouse model of Alzheimer's disease. Neurobiology of Aging 33: 196.e29-40, 2012.

Jiang Q, Lee CYD, Mandrekar S, Wilkinson B, Cramer P, Zelcer N, Mann K, Lamb B, Willson TM, Collins JL, Richardson JC, Smith JD, Comery TA, Riddell D, Holtzman DM, Tontonoz P, Landreth GE. ApoE promotes the proteolytic degradation of Abeta. Neuron 58: 681-93, 2008.

Kafitz KW, Meier SD, Stephan J, Rose CR. Developmental profile and properties of sulforhodamine 101--Labeled glial cells in acute brain slices of rat hippocampus. Journal of Neuroscience Methods 169: 84-92, 2008.

Kang J, Rivest S. Lipid metabolism and neuroinflammation in Alzheimer's disease: a role for liver X receptors. Endocrine Reviews 33: 715-746, 2012. 
Karram K, S, Goebbels S, Goebbels R, Schwab M, Jennissen K, Seifert G, Steinhäuser C, Nave K-A, Trotter J. NG2-expressing cells in the nervous system revealed by the NG2-EYFP-knockin mouse. Genesis 46: 743-57, 2008.

Karran E, Mercken M, De Strooper B. The amyloid cascade hypothesis for Alzheimer's disease: an appraisal for the development of therapeutics. Nature Reviews. Drug Discovery 10: 698, 2011.

Katsouri L, Georgopoulos S. Lack of LDL receptor enhances amyloid deposition and decreases glial response in an Alzheimer's disease mouse model. PloS one 6: e21880, 2011.

Keren-Shaul H, Spinrad A, Weiner A, Matcovitch-Natan O, Dvir-Szternfeld R, Ulland TK, David E, Baruch K, Lara-Astaiso D, Toth B, Itzkovitz S, Colonna M, Schwartz M, Amit I. A Unique Microglia Type Associated with Restricting Development of Alzheimer's Disease. Cell 169: 1276-1290.e17, 2017.

Koistinaho M, Lin S, Wu X, Esterman M, Koger D, Hanson J, Higgs R, Liu F, Malkani S, Bales KR, Paul SM. Apolipoprotein E promotes astrocyte colocalization and degradation of deposited amyloid-beta peptides. Nature Medicine 10: 719-726, 2004.

Koldamova R, Staufenbiel M, Lefterov I. Lack of ABCA1 considerably decreases brain ApoE level and increases amyloid deposition in APP23 mice. Journal of Biological Chemistry 280: 43224-43235, 2005.

Lowry $\mathrm{OH}$, Rosebrough NJ, Farr AL, Randall RJ. Protein measurement with the Folin phenol reagent. Journal of Biological Chemistry 193: 265-275, 1951.

LaDu MJ, Gilligan SM, Lukens JR, Cabana VG, Reardon CA, Van Eldik LJ, Holtzman DM. Nascent astrocyte particles differ from lipoproteins in CSF. Journal of Neurochemistry 70: 2070-2081, 1998.

Laemmli UK. Cleavage of structural proteins during the assembly of the head of bacteriophage T4. Nature 227: 680-685, 1970.

Landel V, Baranger K, Virard I, Loriod B, Khrestchatisky M, Rivera S, Benech P, Féron $\mathrm{F}$. Temporal gene profiling of the 5XFAD transgenic mouse model highlights the importance of microglial activation in Alzheimer's disease. Molecular Neurodegeneration 9: 33, 2014.

Levy-Lahad E, Wasco W, Poorkaj P, Romano DM, Oshima J, Pettingell WH, Yu CE, Jondro PD, Schmidt SD, Wang K. Candidate gene for the chromosome 1 familial Alzheimer's disease locus. Science 269: 973-977, 1995.

Li Y, Cam J, Bu G. Low-density lipoprotein receptor family: endocytosis and signal transduction. Molecular Neurobiology 23: 53-67, 2001.

MacFarlane SN, Sontheimer H. Electrophysiological changes that accompany reactive gliosis in vitro. The Journal of Neuroscience 17: 7316-7329, 1997.

Madisen L, Zwingman TA, Sunkin SM, Oh SW, Zariwala HA, Gu H, Ng LL, Palmiter $\mathrm{RD}$, Hawrylycz MJ, Jones AR, Lein ES, Zeng $\mathrm{H}$. A robust and high-throughput Cre 
reporting and characterization system for the whole mouse brain. Nature Neuroscience 13: 133-40, 2010.

Marquer C, Devauges V, Cossec J-C, Liot G, Lécart S, Saudou F, Duyckaerts C, Lévêque-Fort S, Potier M-C. Local cholesterol increase triggers amyloid precursor protein-Bace1 clustering in lipid rafts and rapid endocytosis. The FASEB Journal 25: 1295-1305, 2011.

Marsh SE, Abud EM, Lakatos A, Karimzadeh A, Yeung ST, Davtyan H, Fote GM, Lau L, Weinger JG, Lane TE, Inlay MA, Poon WW, Blurton-Jones M. The adaptive immune system restrains Alzheimer's disease pathogenesis by modulating microglial function. Proceedings of the National Academy of Sciences 113: E131625, 2016.

Mathiisen TM, Lehre KP, Danbolt NC, Ottersen OP. The perivascular astroglial sheath provides a complete covering of the brain microvessels: An electron microscopic 3D reconstruction. Glia 85:1094-1103, 2010.

Matsuda M, Korn BS, Hammer RE, Moon YA, Komuro R, Horton JD, Goldstein JL, Brown MS, Shimomura I. SREBP cleavage-activating protein (SCAP) is required for increased lipid synthesis in liver induced by cholesterol deprivation and insulin elevation. Genes \& Development 15: 1206-1216, 2001.

Mauch DH, Nägler K, Schumacher S, Göritz C, Müller EC, Otto A, Pfrieger FW. CNS synaptogenesis promoted by glia-derived cholesterol. Science 294: 1354-1357, 2001.

Maulik M, Westaway D, Jhamandas JH, Kar S. Role of cholesterol in APP metabolism and its significance in Alzheimer's disease pathogenesis. Molecular Neurobiology 47: 37-63, 2013.

McAlpine FE, Lee J-K, Harms AS, Ruhn KA, Blurton-Jones M, Hong J, Das P, Golde TE, LaFerla FM, Oddo S, Blesch A, Tansey MG. Inhibition of soluble TNF signaling in a mouse model of Alzheimer's disease prevents pre-plaque amyloid-associated neuropathology. Neurobiology of Disease 34: 163-177, 2009.

McCarthy GF, Leblond CP. Radioautographic evidence for slow astrocyte turnover and modest oligodendrocyte production in the corpus callosum of adult mice infused with 3H-thymidine. The Journal of Comparative Neurology 271: 589-603, 1988.

McDonald JM, Cairns NJ, Taylor-Reinwald L, Holtzman D, Walsh DM. The levels of water-soluble and triton-soluble $A \beta$ are increased in Alzheimer's disease brain. Brain Research 1450: 138-147, 2012.

Minichiello L, Korte M, Wolfer D, Kühn R, Unsicker K, Cestari V, Rossi-Arnaud C, Lipp HP, Bonhoeffer T, Klein R. Essential role for TrkB receptors in hippocampusmediated learning. Neuron 24: 401-14, 1999.

Mori T, Tanaka K, Buffo A, Wurst W, Kühn R, Götz M. Inducible gene deletion in astroglia and radial glia--a valuable tool for functional and lineage analysis. Glia 54: 21-34, 2006. 
Nieweg K, Schaller H, Pfrieger FW. Marked differences in cholesterol synthesis between neurons and glial cells from postnatal rats. Journal of Neurochemistry 109: 125-34, 2009.

Nimmerjahn A, Kirchhoff F, Kerr JND, Helmchen F. Sulforhodamine 101 as a specific marker of astroglia in the neocortex in vivo. Nature Methods 1:31-7, 2004.

Oakley H, Cole SL, Logan S, Maus E, Shao P, Craft J, Guillozet-Bongaarts A, Ohno M, Disterhoft J, van Eldik L, Berry R, Vassar R. Intraneuronal beta-amyloid aggregates, neurodegeneration, and neuron loss in transgenic mice with five familial Alzheimer's disease mutations: potential factors in amyloid plaque formation. The Journal of Neuroscience 26: 10129-40, 2006.

Ofengeim D, Mazzitelli S, Ito Y, DeWitt JP, Mifflin L, Zou C, Das S, Adiconis X, Chen $\mathrm{H}$, Zhu H, Kelliher MA, Levin JZ, Yuan J. RIPK1 mediates a disease-associated microglial response in Alzheimer's disease. Proceedings of the National Academy of Sciences 114: E8788-E8797, 2017.

Pasinetti GM, Johnson SA, Oda T, Rozovsky I, Finch CE. Clusterin (SGP-2): a multifunctional glycoprotein with regional expression in astrocytes and neurons of the adult rat brain. The Journal of Comparative Neurology 339: 387-400, 1994.

Pfrieger FW, Ungerer N. Cholesterol metabolism in neurons and astrocytes. Progress in Lipid Research 50: 357-71, 2011.

Pfrieger FW. Outsourcing in the brain: do neurons depend on cholesterol delivery by astrocytes? BioEssays 25: 72-8, 2003.

Py NA, Bonnet AE, Bernard A, Marchalant Y, Charrat E, Checler F, Khrestchatisky $M$, Rivera S. Differential spatio-temporal regulation of MMPs in the 5xFAD mouse model of Alzheimer's disease: evidence for a pro-amyloidogenic role of MT1-MMP. Frontiers in Aging Neuroscience 6: 247, 2014.

Reitz C, Brayne C, Mayeux R. Epidemiology of Alzheimer disease. Nature Reviews. Neurology 7: 137-152, 2011.

Rivers LE, Young KM, Rizzi M, Jamen F, Psachoulia K, Wade A, Kessaris N, Richardson WD. PDGFRA/NG2 glia generate myelinating oligodendrocytes and piriform projection neurons in adult mice. Nature Neuroscience 11: 1392-1401, 2008.

Rogaev El, Sherrington R, Rogaeva EA, Levesque G, Ikeda M, Liang Y, Chi H, Lin C, Holman K, Tsuda T. Familial Alzheimer's disease in kindreds with missense mutations in a gene on chromosome 1 related to the Alzheimer's disease type 3 gene. Nature 376: 775-778, 1995.

Rome S, Lecomte V, Meugnier E, Rieusset J, Debard C, Euthine V, Vidal H, Lefai E. Microarray analyses of SREBP-1a and SREBP-1c target genes identify new regulatory pathways in muscle. Physiological Genomics 34: 327-337, 2008.

Rone MB, Fan J, Papadopoulos V. Cholesterol transport in steroid biosynthesis: role of protein-protein interactions and implications in disease states. Biochimica et Biophysica Acta 1791: 646-658, 2009. 
Russell DW. Oxysterol biosynthetic enzymes. Biochimica et Biophysica Acta 1529: 126-135, 2000.

Saab AS, Tzvetavona ID, Trevisiol A, Baltan S, Dibaj P, Kusch K, Möbius W, Goetze B, Jahn HM, Huang W, Steffens H, Schomburg ED, Pérez-Samartín A, Pérez-Cerdá F, Bakhtiari D, Matute C, Löwel S, Griesinger C, Hirrlinger J, Kirchhoff F, Nave K-A. Oligodendroglial NMDA Receptors Regulate Glucose Import and Axonal Energy Metabolism. Neuron 91: 119-132, 2016.

Saeed AA, Genové G, Li T, Lütjohann D, Olin M, Mast N, Pikuleva IA, Crick P, Wang Y, Griffiths W, Betsholtz C, Björkhem I. Effects of a disrupted blood-brain barrier on cholesterol homeostasis in the brain. The Journal of Biological Chemistry 289: 23712-22, 2014.

Saher G, Brügger B, Lappe-Siefke C, Möbius W, Tozawa R-I, Wehr MC, Wiel F, Wieland F, Ishibashi S, Nave K-A. High cholesterol level is essential for myelin membrane growth. Nature Neuroscience 8: 468-75, 2005.

Saher G, Quintes S, Nave K-A. Cholesterol: a novel regulatory role in myelin formation. The Neuroscientist 17: 79-93, 2011.

Saher G, Rudolphi F, Corthals K, Ruhwedel T, Schmidt K-F, Löwel S, Dibaj P, Barrette B, Möbius W, Nave K-A. Therapy of Pelizaeus-Merzbacher disease in mice by feeding a cholesterol-enriched diet. Nature Medicine 18: 1130-5, 2012.

Saher G, Stumpf SK. Cholesterol in myelin biogenesis and hypomyelinating disorders. Biochimica et Biophysica Acta 1851 (8): 1083-94, 2015.

Saido T, Leissring MA. Proteolytic degradation of amyloid $\beta$-protein. Cold Spring Harbor Perspectives in Medicine 2: a006379, 2012.

Saito K, Dubreuil V, Arai Y, Wilsch-Bräuninger M, Schwudke D, Saher G, Miyata T, Breier G, Thiele C, Shevchenko A, Nave K-A, Huttner WB. Ablation of cholesterol biosynthesis in neural stem cells increases their VEGF expression and angiogenesis but causes neuron apoptosis. Proceedings of the National Academy of Sciences of the United States of America 106: 8350-5, 2009.

Sauer B. Site-specific recombination: developments and applications. Current Opinion in Biotechnology 5: 521-527, 1994.

Schneider A, Rajendran L, Honsho M, Gralle M, Donnert G, Wouters F, Hell SW, Simons M. Flotillin-Dependent Clustering of the Amyloid Precursor Protein Regulates Its Endocytosis and Amyloidogenic Processing in Neurons. The Journal of Neuroscience 28: 2874-2882, 2008.

Schnell C, Hagos Y, Hülsmann S. Active sulforhodamine 101 uptake into hippocampal astrocytes. PloS one 7: e49398, 2012.

Schnell C, Shahmoradi A, Wichert SP, Mayerl S, Hagos Y, Heuer H, Rossner MJ, Hülsmann S. The multispecific thyroid hormone transporter OATP1C1 mediates cell-specific sulforhodamine 101-labeling of hippocampal astrocytes. Brain Structure \& Function 220(1): 193-203, 2015. 
Schägger H. Tricine-SDS-PAGE. Nature Protocols 1: 16-22, 2006.

Shepardson NE, Shankar GM, Selkoe DJ. Cholesterol level and statin use in Alzheimer disease: II. Review of human trials and recommendations. Archives of Neurology 68: 1385-1392, 2011.

Sherrington R, Rogaev El, Liang Y, Rogaeva EA, Levesque G, Ikeda M, Chi H, Lin C, Li G, Holman K, Tsuda T, Mar L, Foncin JF, Bruni AC, Montesi MP, Sorbi S, Rainero I, Pinessi L, Nee L, Chumakov I, Pollen D, Brookes A, Sanseau P, Polinsky RJ, Wasco W, Da Silva HA, Haines JL, Perkicak-Vance MA, Tanzi RE, Roses AD, Fraser PE, Rommens JM, George-Hyslop PHS. Cloning of a gene bearing missense mutations in early-onset familial Alzheimer's disease. Nature 375: 754-760, 1995.

Simons M, Keller P, de Strooper B, Beyreuther K, Dotti CG, Simons K. Cholesterol depletion inhibits the generation of beta-amyloid in hippocampal neurons. Proceedings of the National Academy of Sciences of the United States of America 95: 6460-4, 1998.

Sinha S, Anderson JP, Barbour R, Basi GS, Caccavello R, Davis D, Doan M, Dovey HF, Frigon N, Hong J, Jacobson-Croak K, Jewett N, Keim P, Knops J, Lieberburg I, Power M, Tan H, Tatsuno G, Tung J, Schenk D, Seubert P, Suomensaari SM, Wang S, Walker D, Zhao J, McConlogue L, John V. Purification and cloning of amyloid precursor protein beta-secretase from human brain. Nature 402: 537-540, 1999.

Slezak M, Göritz C, Niemiec A, Frisén J, Chambon P, Metzger D, Pfrieger FW. Transgenic mice for conditional gene manipulation in astroglial cells. Glia 55: 156576, 2007.

Smart I, Leblond CP. Evidence for division and transformations of neuroglia cells in the mouse brain, as derived from radioautography after injection of thymidine-H3. The Journal of Comparative Neurology 116: 349-367, 1961.

Smith ME. The turnover of myelin in the adult rat. Biochimica et Biophysica Acta 164: 285-293, 1968.

Solito E, Sastre M. Microglia function in Alzheimer's disease. Frontiers in Pharmacology 3: 14, 2012.

Srinivas S, Watanabe T, Lin CS, William CM, Tanabe Y, Jessell TM, Costantini F. Cre reporter strains produced by targeted insertion of EYFP and ECFP into the ROSA26 locus. BMC Developmental Biology 1: 4, 2001.

Srinivasan R, Lu T-Y, Chai H, Xu J, Huang BS, Golshani P, Coppola G, Khakh BS. New Transgenic Mouse Lines for Selectively Targeting Astrocytes and Studying Calcium Signals in Astrocyte Processes In Situ and In Vivo. Neuron 92: 1181-1195, 2016.

Storck SE, Meister S, Nahrath J, Meißner JN, Schubert N, Di Spiezio A, Baches S, Vandenbroucke RE, Bouter Y, Prikulis I, Korth C, Weggen S, Heimann A, Schwaninger M, Bayer TA, Pietrzik CU. Endothelial LRP1 transports amyloid- $\beta(1-$ 42) across the blood-brain barrier. The Journal of Clinical Investigation 126: 123136, 2016. 
Sun X, He G, Song W. BACE2, as a novel APP theta-secretase, is not responsible for the pathogenesis of Alzheimer's disease in Down syndrome. The FASEB Journal 20: 1369-1376, 2006.

Sérougne-Gautheron C, Chevallier F. Time course of biosynthetic cholesterol in the adult rat brain. Biochimica et Biophysica Acta 316: 244-250, 1973.

Tai LM, Youmans KL, Jungbauer L, Yu C, Ladu MJ. Introducing Human APOE into A $\beta$ Transgenic Mouse Models. International Journal of Alzheimer's Disease 2011: 810981, 2011.

Tarawneh R, Holtzman DM. The clinical problem of symptomatic Alzheimer disease and mild cognitive impairment. Cold Spring Harbor Perspectives in Medicine 2: a006148, 2012.

Ulrich JD, Ulland TK, Colonna M, Holtzman DM. Elucidating the Role of TREM2 in Alzheimer's Disease. Neuron 94: 237-248, 2017.

Vallejo M. PACAP signaling to DREAM: a cAMP-dependent pathway that regulates cortical astrogliogenesis. Molecular Neurobiology 39: 90-100, 2009.

Vance JE, Hayashi H. Formation and function of apolipoprotein E-containing lipoproteins in the nervous system. Biochimica et Biophysica Acta 1801: 806-818, 2010.

Vassar R, Bennett BD, Babu-Khan S, Kahn S, Mendiaz EA, Denis P, Teplow DB, Ross S, Amarante P, Loeloff R, Luo Y, Fisher S, Fuller J, Edenson S, Lile J, Jarosinski MA, Biere AL, Curran E, Burgess T, Louis JC, Collins F, Treanor J, Rogers G, Citron M. Beta-secretase cleavage of Alzheimer's amyloid precursor protein by the transmembrane aspartic protease BACE. Science 286: 735-741, 1999.

Veeraraghavalu K, Zhang C, Miller S, Hefendehl JK, Rajapaksha TW, Ulrich J, Jucker M, Holtzman DM, Tanzi RE, Vassar R, Sisodia SS. Comment on "ApoEdirected therapeutics rapidly clear $\beta$-amyloid and reverse deficits in AD mouse models". Science 340: 924-f, 2013.

Verghese PB, Castellano JM, Garai K, Wang Y, Jiang H, Shah A, Bu G, Frieden C, Holtzman DM. ApoE influences amyloid- $\beta$ ( $A \beta$ ) clearance despite minimal apoE/A $\beta$ association in physiological conditions. Proceedings of the National Academy of Sciences of the United States of America 110: E1807-16, 2013.

Wahrle S, Das P, Nyborg AC, McLendon C, Shoji M, Kawarabayashi T, Younkin LH, Younkin SG, Golde TE. Cholesterol-dependent gamma-secretase activity in buoyant cholesterol-rich membrane microdomains. Neurobiology of Disease 9: 1123, 2002.

Wahrle SE, Jiang H, Parsadanian M, Hartman RE, Bales KR, Paul SM, Holtzman DM. Deletion of Abca1 increases Abeta deposition in the PDAPP transgenic mouse model of Alzheimer disease. The Journal of Biological Chemistry 280: 4323643242, 2005. 
Wahrle SE, Jiang $H$, Parsadanian M, Kim J, Li A, Knoten A, Jain S, HirschReinshagen V, Wellington CL, Bales KR, Paul SM, Holtzman DM. Overexpression of ABCA1 reduces amyloid deposition in the PDAPP mouse model of Alzheimer disease. The Journal of Clinical Investigation 118: 671, 2008.

Wang C-Y, Liu P-Y, Liao JK. Pleiotropic effects of statin therapy: molecular mechanisms and clinical results. Trends in Molecular Medicine 14: 37-44, 2007.

Wang DD, Bordey A. The astrocyte odyssey. Progress in Neurobiology 86: 342-67, 2008.

Wang Y, Cella M, Mallinson K, Ulrich JD, Young KL, Robinette ML, Gilfillan S, Krishnan GM, Sudhakar S, Zinselmeyer BH, Holtzman DM, Cirrito JR, Colonna M. TREM2 lipid sensing sustains the microglial response in an Alzheimer's disease model. Cell 160: 1061-71, 2015.

Wang Y, Rogers PM, Su C, Varga G, Stayrook KR, Burris TP. Regulation of cholesterologenesis by the oxysterol receptor, LXRalpha. Journal of Biological Chemistry 283: 26332-26339, 2008.

Wang Y, Ulland TK, Ulrich JD, Song W, Tzaferis JA, Hole JT, Yuan P, Mahan TE, Shi Y, Gilfillan S, Cella M, Grutzendler J, DeMattos RB, Cirrito JR, Holtzman DM, Colonna M. TREM2-mediated early microglial response limits diffusion and toxicity of amyloid plaques. The Journal of Experimental Medicine 213: 667-675, 2016.

Winchenbach J, Düking T, Berghoff SA, Stumpf SK, Hülsmann S, Nave KA, Saher G. Inducibile targeting of CNS astrocyte in Aldh1l1-CreERT2 BAC transgenic mice. F1000Research 5:2934, 2016.

Xie C, Lund EG, Turley SD, Russell DW, Dietschy JM. Quantitation of two pathways for cholesterol excretion from the brain in normal mice and mice with neurodegeneration. Journal of Lipid Research 44: 1780-1789, 2003.

Yan R, Bienkowski MJ, Shuck ME, Miao H, Tory MC, Pauley AM, Brashier JR, Stratman NC, Mathews WR, Buhl AE, Carter DB, Tomasselli AG, Parodi LA, Heinrikson RL, Gurney ME. Membrane-anchored aspartyl protease with Alzheimer's disease beta-secretase activity. Nature 402: 533-537, 1999.

Yan R, Munzner JB, Shuck ME, Bienkowski MJ. BACE2 functions as an alternative alpha-secretase in cells. Journal of Biological Chemistry 276: 34019-34027, 2001.

Yang Y, Vidensky S, Jin L, Jie C, Lorenzini I, Frankl M, Rothstein JD. Molecular comparison of GLT1+ and ALDH1L1+ astrocytes in vivo in astroglial reporter mice. Glia 59: 200-7, 2011.

Yeh FL, Wang Y, Tom I, Gonzalez LC, Sheng M. TREM2 Binds to Apolipoproteins, Including APOE and CLU/APOJ, and Thereby Facilitates Uptake of Amyloid-Beta by Microglia. Neuron 91: 328-340, 2016.

Young KM, Psachoulia K, Tripathi RB, Dunn S-J, Cossell L, Attwell D, Tohyama K, Richardson WD. Oligodendrocyte dynamics in the healthy adult CNS: evidence for myelin remodeling. Neuron 77: 873-885, 2013. 
Zani IA, Stephen SL, Mughal NA, Russell D, Homer-Vanniasinkam S, Wheatcroft $\mathrm{SB}$, Ponnambalam S. Scavenger receptor structure and function in health and disease. Cells 4: 178-201, 2015.

Zhang Y, Chen K, Sloan SA, Bennett ML, Scholze AR, O'Keeffe S, Phatnani HP, Guarnieri P, Caneda C, Ruderisch N, Deng S, Liddelow SA, Zhang C, Daneman R, Maniatis T, Ben Barres A, Wu JQ. An RNA-Sequencing Transcriptome and Splicing Database of Glia, Neurons, and Vascular Cells of the Cerebral Cortex. The Journal of Neuroscience 34: 11929-47, 2014.

Zhao J, Fu Y, Yasvoina M, Shao P, Hitt B, O'Connor T, Logan S, Maus E, Citron M, Berry R, Binder L, Vassar R. Beta-site amyloid precursor protein cleaving enzyme 1 levels become elevated in neurons around amyloid plaques: implications for Alzheimer's disease pathogenesis. The Journal of Neuroscience 27: 3639-3649, 2007.

Zhao Z, Nelson AR, Betsholtz C, Zlokovic BV. Establishment and Dysfunction of the Blood-Brain Barrier. Cell 163: 1064-1078, 2015.

van Deijk A-LF, Camargo N, Timmerman J, Heistek T, Brouwers JF, Mogavero F, Mansvelder HD, Smit AB, Verheijen MHG. Astrocyte lipid metabolism is critical for synapse development and function in vivo. Glia 65: 670-682, 2017. 